Article

\title{
Imidazo[1,2-a]quinoxalines Derivatives Grafted with Amino Acids: Synthesis and Evaluation on A375 Melanoma Cells
}

\author{
Adrien Chouchou ${ }^{1,+}$, Cindy Patinote ${ }^{1,2,+}$, Pierre Cuq ${ }^{1}$, Pierre-Antoine Bonnet ${ }^{1, *}$ \\ and Carine Deleuze-Masquéfa ${ }^{1}$ \\ 1 IBMM, Université de Montpellier, CNRS, ENSCM, 34000 Montpellier, France; \\ adrien.chouchou@umontpellier.fr (A.C.); cindy.patinote@umontpellier.fr (C.P.); \\ pierre.cuq@umontpellier.fr (P.C.); carine.masquefa@umontpellier.fr (C.D.-M.) \\ 2 Société d'Accélération du Transfert de Technologies (SATT AxLR), CSU, 950 rue Saint Priest, \\ 34090 Montpellier, France \\ * Correspondence: pierre-antoine.bonnet@umontpellier.fr; Tel.: +33-4-11-75-95-41 \\ + These authors contributed equally to this work.
}

Received: 30 October 2018; Accepted: 13 November 2018; Published: 15 November 2018

check for updates

\begin{abstract}
Imiqualines (imidazoquinoxaline derivatives) are anticancer compounds with high cytotoxic activities on melanoma cell lines. The first generation of imiqualines, with two lead compounds (EAPB0203 and EAPB0503), shows remarkable in vitro ( $\mathrm{IC}_{50}=1570 \mathrm{nM}$ and $\mathrm{IC}_{50}=200 \mathrm{nM}$, respectively, on the A375 melanoma cell line) and in vivo activity on melanoma xenografts. The second generation derivatives, EAPB02302 and EAPB02303, are more active, with $\mathrm{IC}_{50}=60 \mathrm{nM}$ and $\mathrm{IC}_{50}=10 \mathrm{nM}$, respectively, on A375 melanoma cell line. The aim of this study was to optimize the bioavailability of imiqualine derivatives, without losing their intrinsic activity. For that, we achieved chemical modulation on the second generation of imiqualines by conjugating amino acids on position 4. A new series of twenty-five compounds was efficiently synthesized by using microwave assistance and tested for its activity on the A375 cell line. In the new series, compounds 11a, 9d and $\mathbf{1 1 b}$ show cytotoxic activities less than second generation compounds, but similar to that of the first generation ones $\left(\mathrm{IC}_{50}=403 \mathrm{nM}, \mathrm{IC}_{50}=128 \mathrm{nM}\right.$ and $\mathrm{IC}_{50}=584 \mathrm{nM}$, respectively). The presence of an amino acid leads to significant enhancement of the water solubility for improved drugability.
\end{abstract}

Keywords: imidazo[1,2-a]quinoxaline; melanoma; imiqualine; A375 structure-activity relationship

\section{Introduction}

Cutaneous melanoma is a malignant tumour of melanocytes located in the basal epidermis. It is the most aggressive and lethal form of skin cancer because of its fast-metastatic development [1,2]. Its incidence has been increasing worldwide for several decades. While in its early stages remarkable outcomes can be achieved with surgery alone, metastatic melanoma requires therapeutic treatment intervention. Thanks to genomic studies, knowledge regarding the molecular biology of melanomas has improved, leading to recent FDA-approved therapies [3-5]. However, all the subtypes of the disease are not equally treated [5] and marked resistance mostly against kinase inhibitors rapidly occurs [6,7]. As the incidence among the worldwide fair-skinned population is increasing, this public health concern remains challenging.

Our group is working on the development of the imidazo[1,2-a]quinoxaline derivatives presented in Scheme 1, called imiqualines, as potential antitumoral agents, particularly for the treatment of melanoma [8,9]. The first generation of imiqualines was essentially substituted on position 1 by multiple aromatic moieties directly grafted to the main structure or via an alkyl linker. The second 
generation is characterized by the presence of the 3,4-dihydroxyphenyl moiety on position 1 since the presence of such catechol residue enhances global hydrophilicity. The chemical modulations of the first hits, EAPB0203 and EAPB0503, afforded new leads EAPB02303 and its N-demethylated derivative EAPB02302, with impressive in vitro activities in the nanomolar range on the A375 human melanoma cancer cell line $[10,11]$.

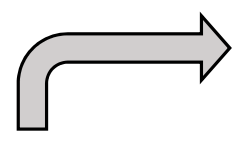<smiles>[R2]Nc1nc2ccccc2n2c([R])cnc12</smiles>

Imiqualines general structure

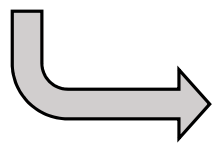

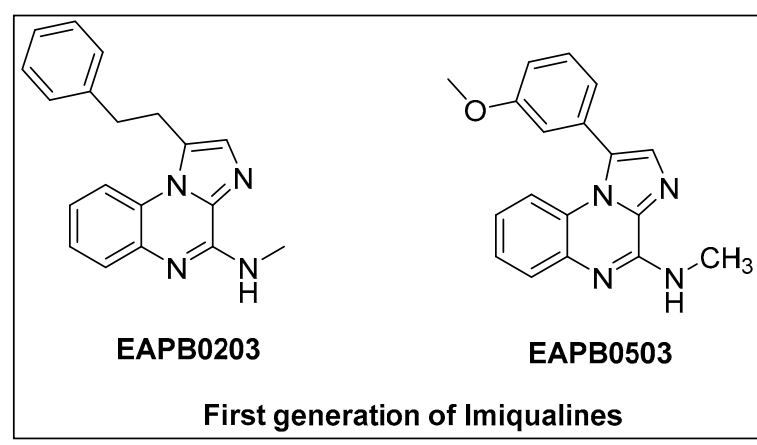

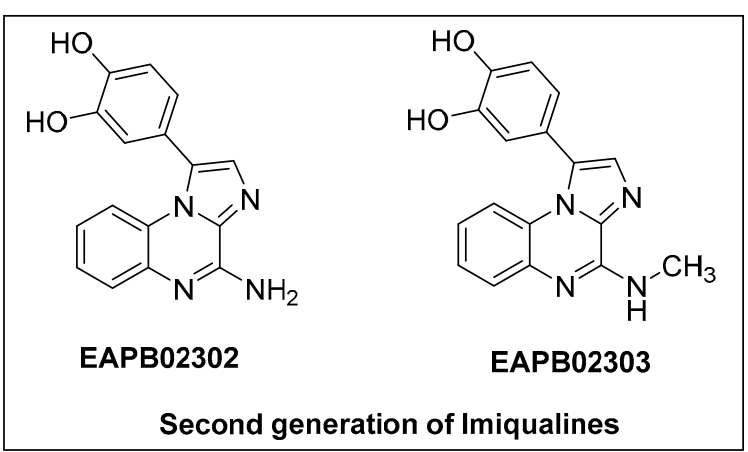

Scheme 1. General and lead compounds structures of the imiqualines.

EAPB02302 and EAPB02303 are considered as promising anticancer agents but exhibit high lipophilicity (cLogP values estimated at 2.68 and 3.55, respectively), which might be critical for future preclinical in vivo studies. Indeed, their low solubility in water might be a major drawback for further development, especially in the case of intravenous use. In a preliminary study [12], the pharmacokinetic parameters of EAPB02303 were determined in mice after a single intraperitoneal administration. For this, the compound was solubilized in a mixture of DMSO, Tween 80 and sodium chloride solution $0.9 \%$ $(10 / 10 / 80, v / v / v)$. The use of DMSO is recognized as toxic [13], in particular when used repeatedly as would be the case in an efficacy study. In order to optimize the results of efficacy studies, we chose to chemically modulate our lead compounds to obtain more soluble compounds with a moderate impact on the cytotoxic activity. The result of the introduction of various amino acids on position 4 of the heterocycle on both the physicochemical properties and biological activity was studied. Such an approach to increase solubility, which remains a key factor for potential pharmaceutical development, has already been described in the literature [14-17]. The introduction of amino acid moieties has been showed to increase the water solubility as well as selective cytotoxicity [18-20]. We present herein the synthesis of new imidazo[1,2-a]quinoxalines decorated with a panel of natural $\alpha$-amino acids and their in vitro preliminary evaluation on A375 cell line.

\section{Results and Discussion}

\subsection{Synthesis of Imidazo[1,2-a]quinoxaline Derivatives}

The synthetic pathways and the structures of imidazo[1,2-a]quinoxalines used in this study are given in Schemes 2 and 3. Intermediates $\mathbf{1}$ to $\mathbf{5}$ were synthetized thanks to a route we previously described $[9,21]$. Briefly, the carbonylimidazole dimer 2 results from the condensation of the 
2-imidazole carboxylic acid 1 in presence of thionyl chloride. Addition of O-fluoroaniline to the dimer 2 gives the intermediate 3 . Cyclisation is facilitated by using sodium hydride in dimethylacetamide. Treatment of compound 4 with phosphorus oxychloride and $N, N$-diethylaniline gives the chlorinated key intermediate 5 .
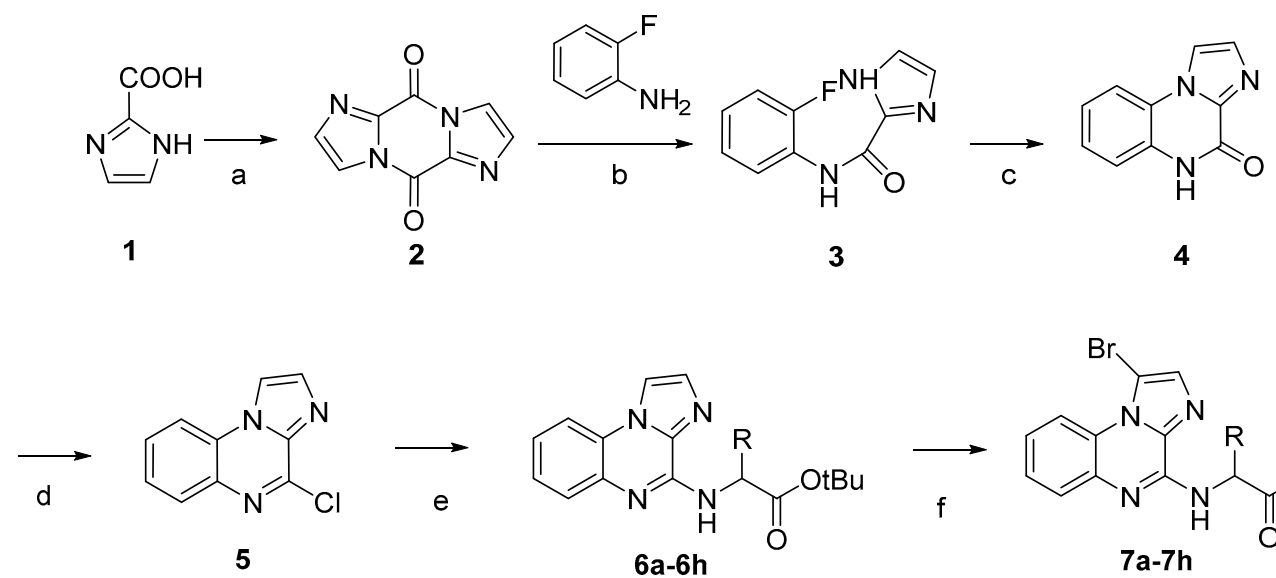<smiles>[R]C(Nc1nc2ccccc2n2c(Br)cnc12)C(=O)OCC</smiles>

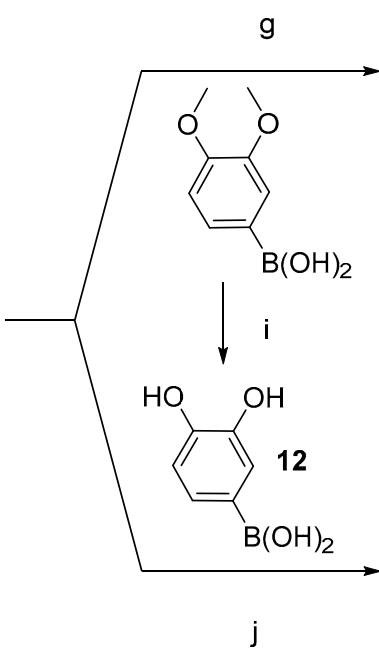<smiles>[R]C(Nc1nc2ccccc2n2c(-c3ccc(OC)c(OC)c3)cnc12)C(=O)OCCCCC(C)C(C)C</smiles><smiles>[R]C(Nc1nc2ccccc2n2c(-c3ccc(O)c(O)c3)cnc12)C(=O)OCC</smiles><smiles>[R]C(Nc1nc2ccccc2n2c(-c3ccc(O)c(O)c3)cnc12)C(=O)O</smiles>

Scheme 2. Synthesis of imidazo[1,2-a]quinoxaline derivatives grafted with the $\alpha$-amine group of the amino acid. Reagents and Conditions: (a) $\mathrm{SOCl}_{2}$, reflux, 18h; (b) NaHMDS, THF, $0{ }^{\circ} \mathrm{C}$ to RT, $5 \mathrm{~h}$; (c) $\mathrm{NaH}, \mathrm{DMA}$, reflux, 48 h; (d) DEA, $\mathrm{POCl}_{3}, \mathrm{MW}\left(130^{\circ} \mathrm{C}, 15 \mathrm{~min}\right)$; (e) H-amino acid (PG)-OtBu, DIEA, DMF, MW (150 $\left.{ }^{\circ} \mathrm{C}, 30-60 \mathrm{~min}\right)$; (f) NBS, $\mathrm{CHCl}_{3}$, reflux, $2 \mathrm{~h}$; (g) 3,4-dimethoxyphenylboronic acid, $\mathrm{Pd}\left(\mathrm{PPh}_{3}\right)_{4}, \mathrm{Na}_{2} \mathrm{CO}_{3}, \mathrm{DME}, \mathrm{MW}\left(140^{\circ} \mathrm{C}, 20 \mathrm{~min}\right)$; (h) $\mathrm{BBr}_{3}, \mathrm{CH}_{2} \mathrm{Cl}_{2}, \mathrm{RT}, 1 \mathrm{~h}-3 \mathrm{~h},(\mathbf{i}) \mathrm{BBr}_{3}, \mathrm{CH}_{2} \mathrm{Cl}_{2}, \mathrm{RT}$, 1h; (j) 3,4-dihydroxyphenylboronic acid 12, $\mathrm{Pd}\left(\mathrm{PPh}_{3}\right)_{4}, \mathrm{Na}_{2} \mathrm{CO}_{3}, \mathrm{DME}, \mathrm{MW}\left(140{ }^{\circ} \mathrm{C}, 20 \mathrm{~min}\right)$; (k) TFA/ $\mathrm{CH}_{2} \mathrm{Cl}_{2}(1 / 1, v / v), \mathrm{RT}, 1-2 \mathrm{~h}$.

The chlorine of 5 can be substituted by an amino group in the presence of diisopropylethylamine in DMF under microwave assistance. Among the commercially available amino acids, we chose to study the effect of a short or long, substituted or not, aliphatic or aryl side chain with or without a hydrophilic amino or phenolic group. The amino group could be either the $\alpha$-amine of an amino acid or the amine of the side chain for the ornithine residue. The introduction of HGlyOtBu, $\mathrm{HAlaOtBu}$, $\mathrm{HValOtBu}, \mathrm{HLeuOtBu}, \mathrm{HLysOtBu}, \mathrm{HOrnOtbu}, \mathrm{HPheOtBu}, \mathrm{HTyrOtBu}$ is described in Scheme 2 and the grafting of BocOrnOtBu is depicted in Scheme 3. The bromination of the intermediates 6 by 
N-bromosuccinimide leads to compounds 7. The 3,4-dimethoxyphenyl group is introduced in position 1 via a Suzuki-Miyaura cross-coupling reaction to furnish compounds $\mathbf{8 a - 8 f}$ and $\mathbf{8 i}$. Boron tribromide did not allow the cleavage of all the protections to give the targeted compounds 11a-11i, even if supplementary equivalents of $\mathrm{BBr}_{3}$ were added at the beginning or during the reaction, or if the reaction time was extended. Such an approach allows one to obtain compounds $\mathbf{1 0 b}-\mathbf{1 0 d}$ as the main products. These compounds present remaining methoxy groups on the phenyl on position 1 without the protecting groups on the amino acid. The by-products correspond to one remaining methoxy group on the phenyl either at position $3^{\prime}$ or $4^{\prime}$, but unfortunately, they could not be recovered separately.<smiles>Clc1nc2ccccc2n2ccnc12</smiles>

5<smiles>CCCOC(=O)C(CCCNc1nc2ccccc2n2ccnc12)NC(=O)OCC</smiles><smiles>CCCCOC(=O)NC(CCCNc1nc2ccccc2n2c(Br)cnc12)C(=O)OCCCC</smiles><smiles>CCCCOC(=O)C(CCCNc1nc2ccccc2n2c(-c3ccc(OC)c(OC)c3)cnc12)NC(=O)C(CCCNc1nc2ccccc2n2c(-c3ccc(O)c(O)c3)cnc12)NCCCC(NC(=O)OCCC)C(=O)OCC</smiles>

Scheme 3. Synthesis of the ornithine-containing imidazo[1,2-a]quinoxaline derivative grafted by the side chain. Reagents and Conditions: (e) Boc-Orn-OtBu, $\mathrm{HCl}, \mathrm{DIEA}, \mathrm{DMF}, \mathrm{MW}\left(150{ }^{\circ} \mathrm{C}, 30-60 \mathrm{~min}\right)$; (f) NBS, $\mathrm{CHCl}_{3}$, reflux, $2 \mathrm{~h}$; (g) 3,4-dimethoxyphenylboronic acid, $\mathrm{Pd}\left(\mathrm{PPh}_{3}\right)_{4}, \mathrm{Na}_{2} \mathrm{CO}_{3}, \mathrm{DME}, \mathrm{MW}$ (140 ${ }^{\circ} \mathrm{C}, 20 \mathrm{~min}$ ); (h) $\mathrm{BBr}_{3}, \mathrm{CH}_{2} \mathrm{Cl}_{2}, \mathrm{RT}, 1 \mathrm{~h}-3 \mathrm{~h}$; (i) $\mathrm{BBr}_{3}, \mathrm{CH}_{2} \mathrm{Cl}_{2}, \mathrm{RT}, 1 \mathrm{~h}$; (j) 3,4-dihydroxyphenylboronic acid 12, $\mathrm{Pd}\left(\mathrm{PPh}_{3}\right)_{4}, \mathrm{Na}_{2} \mathrm{CO}_{3}, \mathrm{DME}, \mathrm{MW}\left(140{ }^{\circ} \mathrm{C}, 20 \mathrm{~min}\right)$; (k) TFA $/ \mathrm{CH}_{2} \mathrm{Cl}_{2}(1 / 1, v / v), \mathrm{RT}, 1-2 \mathrm{~h}$.

As target compounds 11a-11f could not be obtained by this way, we decided to deprotect first the 3,4-dimethoxyphenylboronic acid using boron tribromide in order to obtain the 3,4-dihydroxy-phenylboronic acid 12. This boronic acid readily reacts with intermediates 7 under microwave irradiation to furnish the hydroxylated derivatives 9a-9i. A final step of deprotection of the amino acid moiety using TFA in $\mathrm{CH}_{2} \mathrm{Cl}_{2}$ was used to obtain the final compounds 11a-11i. 


\subsection{In Vitro Cytotoxic Activity on A375 Cell Line and Calculated ClogP}

All new imidazo[1,2-a]quinoxaline derivatives $\mathbf{8 b}-\mathbf{d}, \mathbf{8 f}, \mathbf{9 a}-\mathbf{9} \mathbf{i}, \mathbf{1 0 b}-\mathbf{d}$ and $\mathbf{1 1 a}-\mathbf{1 1 i}$ were tested for their in vitro antiproliferative activities on the human melanoma cell line A375. Their $\mathrm{IC}_{50}$ values (concentration of the compound (nM) producing 50\% cell growth inhibition after $96 \mathrm{~h}$ of drug exposure) were determined using in vitro cytotoxicity assays and are displayed in Tables 1 and 2.

Table 1. Synthesis of imidazo[1,2-a]quinoxaline derivatives grafted with the $\alpha$-amine of the amino acid as described in Scheme 2: $\operatorname{cLogP}$, theoretical water solubility $(\mathrm{mg} / \mathrm{mL})$ at $\mathrm{pH} 7.4$ calculated values and $\mathrm{IC}_{50}$ values against A375 (human melanoma cell line).

\begin{tabular}{|c|c|c|c|c|c|c|}
\hline $\begin{array}{c}\text { Amino } \\
\text { Acids }\end{array}$ & $-\mathbf{R}$ & $-\mathbf{R}^{\prime}$ & Compounds & $C \log P^{a}$ & $\begin{array}{l}\text { Theoretical } \\
\text { Water } \\
\text { Solubility } \\
(\mathrm{mg} / \mathrm{mL}) \text { at } \\
\text { pH 7.4 }\end{array}$ & $\begin{array}{c}\mathrm{IC}_{50} \\
\text { Values } \\
{ }^{\mathrm{c}}(\mathrm{nM})\end{array}$ \\
\hline \multirow{4}{*}{ Gly } & \multirow{3}{*}{$-\mathrm{H}$} & & $8 a$ & 5.25 & $5.88 \times 10^{-4}$ & $\mathrm{ND}^{\mathrm{d}}$ \\
\hline & & $-\mathrm{H}$ & $9 a$ & 4.99 & $1.79 \times 10^{-3}$ & 1932 \\
\hline & & & $11 a$ & 2.59 & 47.18 & 403 \\
\hline & \multirow{4}{*}{$-\mathrm{CH}_{3}$} & & $8 b$ & 5.61 & $3.80 \times 10^{-4}$ & $>10,000$ \\
\hline \multirow{3}{*}{ Ala } & & & $9 b$ & 5.34 & $1.15 \times 10^{-3}$ & 6103 \\
\hline & & & $10 b$ & 3.21 & 6.01 & 5947 \\
\hline & & & $11 b$ & 2.94 & 19.92 & 584 \\
\hline \multirow{4}{*}{ Val } & \multirow{3}{*}{$\mathrm{CH}_{3}$} & & $8 c$ & 6.47 & $1.35 \times 10^{-4}$ & $>10,000$ \\
\hline & & & $9 \mathrm{c}$ & 6.21 & $4.07 \times 10^{-4}$ & 7180 \\
\hline & & & $10 c$ & 4.07 & 1.91 & $>10,000$ \\
\hline & & & $11 \mathrm{c}$ & 3.81 & 6.5 & 7166 \\
\hline \multirow{4}{*}{ Leu } & & & $8 d$ & 6.98 & $7.43 \times 10^{-5}$ & \multirow{5}{*}{$\begin{array}{c}>10,000 \\
128 \\
>10,000 \\
838 \\
\text { ND }^{\mathrm{d}}\end{array}$} \\
\hline & & & $9 d$ & 6.72 & $2.23 \times 10^{-4}$ & \\
\hline & & & $10 \mathrm{~d}$ & 4.58 & 0.89 & \\
\hline & & & $11 d$ & 4.32 & 3.08 & \\
\hline \multirow{3}{*}{ Lys } & & & $8 e$ & 7.05 & $5.70 \times 10^{-5}$ & \\
\hline & & & $9 e$ & 6.79 & $1.56 \times 10^{-4}$ & \multirow{3}{*}{$\begin{array}{c}4575 \\
673 \\
>10,000\end{array}$} \\
\hline & & & $11 \mathrm{e}$ & 2.78 & $4.34 \times 10^{-3}$ & \\
\hline \multirow{3}{*}{ Orn } & & & $8 f$ & 7.01 & $3.43 \times 10^{-5}$ & \\
\hline & & & $9 f$ & 6.75 & $9.07 \times 10^{-5}$ & \multirow{3}{*}{$\begin{array}{l}1111 \\
3404 \\
1999\end{array}$} \\
\hline & & & $11 f$ & 2.57 & $5.82 \times 10^{-3}$ & \\
\hline \multirow{2}{*}{ Phe } & & & $9 g$ & 6.82 & $1.06 \times 10^{-4}$ & \\
\hline & & & $11 \mathrm{~g}$ & 3.71 & 6.47 & \multirow{2}{*}{$\begin{array}{c}951 \\
4117\end{array}$} \\
\hline \multirow[t]{2}{*}{ Tyr } & & & $9 \mathrm{~h}$ & 7.99 & $3.31 \times 10^{-5}$ & \\
\hline & & & $11 \mathrm{~h}$ & 3.05 & 10.17 & 2174 \\
\hline
\end{tabular}

${ }^{a}, \mathrm{~b} \operatorname{cLogP}$ and theoretical water solubility $(\mathrm{mg} / \mathrm{mL})$ at $\mathrm{pH} 7.4$ values are calculated using the ACDLabs®software;

${ }^{\mathrm{c}} \mathrm{IC}_{50}$ values, concentration of the compound (nM) producing $50 \%$ cell growth inhibition after $96 \mathrm{~h}$ of drug exposure, as determined by the MTT assay. Each experiment was performed in triplicate, and the results are presented as average values. Coefficients of variation were less than $10 \%{ }^{\mathrm{d}} \mathrm{ND}$ : Not determined. 
Table 2. Synthesis of imidazo[1,2-a]quinoxaline derivatives grafted with the side chain amine of ornithine as described in Scheme 3: ClogP, theoretical water solubility $(\mathrm{mg} / \mathrm{mL})$ at $\mathrm{pH} 7.4$ calculated values and $\mathrm{IC}_{50}$ values against $\mathrm{A} 375$ (human melanoma cell line).

\begin{tabular}{ccccc}
\hline Amino acid & Compounds & ClogP $^{\mathbf{a}}$ & $\begin{array}{c}\text { Theoretical Water Solubility } \\
(\mathbf{m g} / \mathbf{m L}) \text { at } \mathbf{~ p H ~ 7 . 4}\end{array}$ & IC $_{\mathbf{5 0}}$ Values $^{\mathbf{c}}$ (nM) \\
\hline \multirow{2}{*}{ Orn } & $\mathbf{8 i}$ & 6.12 & $1.62 \times 10^{-4}$ & $>10,000$ \\
& $\mathbf{9 i}$ & 5.86 & $4.50 \times 10^{-4}$ & $>10,000$ \\
& $\mathbf{1 1 i}$ & 2.99 & $3.05 \times 10^{-3}$ & 5168 \\
\hline
\end{tabular}

${ }^{\mathrm{a}, \mathrm{b}} \mathrm{C} \log \mathrm{P}$ and theoretical water solubility $(\mathrm{mg} / \mathrm{mL})$ at $\mathrm{pH} 7.4$ values are calculated using the ACDLabs®software. ${ }^{\mathrm{c}} \mathrm{IC}_{50}$ values, concentration of the compound (nM) producing $50 \%$ cell growth inhibition after $96 \mathrm{~h}$ of drug exposure, as determined by the MTT assay. Each experiment was performed in triplicate, and the results are presented as average values. Coefficients of variation were less than $10 \%$.

Lipophilicity, expressed as the logarithm of a compound's octanol/water partition coefficient $(\mathrm{C} \log \mathrm{P})$, is a physicochemical property of drugs that affects many biological mechanisms, especially drug absorption and distribution (absorption, plasma protein binding and membrane permeation) [22]. This parameter can also be correlated to solubility, metabolism and toxicity [23,24]. In a drug discovery process, compounds must be sufficiently lipophilic to cross the membrane barriers and at the same time be sufficiently water soluble to reach their targets. Therefore, a poor water solubility is a common cause of rejection during development [25]. This is why we also estimated the lipophilicity and hydrophilicity properties of all new compounds by predicting their $\mathrm{ClogP}$ and theoretical water solubility values thanks to fragmentation methods available on the ACDLabs ${ }^{\circledR}$ software. These calculated values are purely theoretical but give a good estimate of what might be the solubility of the compounds in blood circulation ( $\mathrm{pH}$ 7.4). This approach is used in pharmaceutical industry for the screening of new compounds $[26,27]$. Results of our new imidazo[1,2-a]quinoxaline derivatives were compared each other as well as with the first and second generation imiqualine leads as shown in Table 3.

Table 3. First and second generation imiqualines leads: $\mathrm{Clog}$, theoretical water solubility $(\mathrm{mg} / \mathrm{mL})$ at pH 7.4 calculated values and IC50 values against A375 (human melanoma cell line).

\begin{tabular}{cccc}
\hline Compounds & ClogP ${ }^{\text {a }}$ & $\begin{array}{c}\text { Theoretical Water Solubility } \\
(\mathbf{m g} / \mathbf{m L}) \text { at } \mathbf{~ p H ~ 7 . 4}\end{array}$ & IC $_{\mathbf{5 0}}$ Values $^{\mathbf{c}}$ (nM) \\
\hline EAPB0203 & 4.6 & $3.46 \times 10^{-3}$ & 1570 \\
EAPB0503 & 4.48 & $2.60 \times 10^{-3}$ & 200 \\
EAPB02302 & 2.68 & $4.28 \times 10^{-2}$ & 60 \\
EAPB02303 & 3.55 & $1.74 \times 10^{-2}$ & 10 \\
\hline
\end{tabular}

${ }_{\mathrm{a}, \mathrm{b}} \mathrm{C} \log \mathrm{P}$ and theoretical water solubility $(\mathrm{mg} / \mathrm{mL})$ at $\mathrm{pH} 7.4$ values are calculated using the ACDLabs®software.

${ }^{\mathrm{c}} \mathrm{IC}_{50}$ values, concentration of the compound (nM) producing $50 \%$ cell growth inhibition after $96 \mathrm{~h}$ of drug exposure, as determined by the MTT assay. Each experiment was performed in triplicate, and the results are presented as average values. Coefficients of variation were less than $10 \%$.

Compounds $\mathbf{8 b}, \mathbf{8 c}, \mathbf{8 d}$ and $\mathbf{8 f}$ have fully protected amino acid residues (side chain and carboxylic function) with 3,4-dimethoxy substitution on the phenyl ring in position 1 . They exhibit weak $\mathrm{IC}_{50}$ values higher than 10,000 $\mathrm{nM}$. Compounds belonging to this chemical series are very lipophilic with estimated $\mathrm{C} \log \mathrm{P}$ values higher than 5 (5.25, 5.61, 6.47, 6.98, 7.05 and 7.01 for compounds $\mathbf{8 a}, \mathbf{8 b}, \mathbf{8 c}$, $\mathbf{8 d}, \mathbf{8 e}$ and $\mathbf{8 f}$, respectively). The theoretical water solubility of compounds $\mathbf{8}$ at $\mathrm{pH} 7.4$ is very low since the order varies from $5.88 \times 10^{-4}$ to $3.43 \times 10^{-5} \mathrm{mg} / \mathrm{mL}$ for compounds $8 \mathbf{a}$ and $\mathbf{8 f}$, respectively. Therefore, the presence of protective groups on the amino acids and the dimethoxy groups on the phenyl appears not to be valuable, both in terms of water solubility and cytotoxic efficiency.

Compounds $9 \mathbf{a}-\mathbf{9 i}$ are only protected on the amino acids moieties. These compounds exhibit various cytotoxic activities with $\mathrm{IC}_{50}$ values ranging from 128 to $7180 \mathrm{nM}$, for $\mathbf{9 d}$ and $9 \mathrm{c}$, respectively. These compounds show high lipophilicity since their $C \log P$ values range from 4.99 to 7.99 for $9 \mathbf{a}$ and $9 \mathrm{~h}$ respectively. Similarly, the theoretical values of water solubility are between $3.31 \times 10^{-5}$ and 
$1.79 \times 10^{-3} \mathrm{mg} / \mathrm{mL}$ for $\mathbf{9 h}$ and $9 \mathrm{a}$, respectively. By comparing compounds $\mathbf{8}$ and $\mathbf{9}$, we note that the replacement of the methoxy groups by the hydroxy groups induces, for some residues, an important increase of the biological activities. Nevertheless, such modification, with conservation of the amino acid protection, does not improve the theoretical water solubility of the new synthesized compounds.

Compounds $\mathbf{1 0 b}-\mathbf{1 0 d}$ are deprotected on the amino acid residues but still present dimethoxy groups on the phenyl substitution at position 1 . These compounds exhibit $\mathrm{IC}_{50}$ values higher than $5000 \mathrm{nM}\left(\mathrm{IC}_{50}\right.$ values at $5947 \mathrm{nM}$ for 10b and higher than 10,000 nM for 10c and 10d). However, ClogP values are below 5 and theoretical water solubilities are higher than $0.8 \mathrm{mg} / \mathrm{mL}$. The transition from compounds $\mathbf{8}$ to compounds $\mathbf{1 0}$ provides a significant decrease of lipophilicity as well as an improvement of theoretical water solubility. The comparison of the compounds according to the grafted residue permits to highlight this improvement. Actually, ClogP values decrease from 5.61 to 3.21 , from 6.47 to 4.07 and from 6.98 to 4.58 for compounds $8 \mathbf{b}$ to $\mathbf{1 0 b}, \mathbf{8 c}$ to $\mathbf{1 0 c}$ and $\mathbf{8 d}$ to $\mathbf{1 0 d}$, respectively. Even more impressive, the theoretical water solubility values increase from $3.8 \times 10^{-4}$ to $6.01,1.35 .10^{-4}$ to 1.91 and $7.43 \times 10^{-5}$ to $0.89 \mathrm{mg} / \mathrm{mL}$ for compounds $\mathbf{8 b}$ to $\mathbf{1 0 b}, \mathbf{8 c}$ to $\mathbf{1 0 c}$ and $8 \mathbf{d}$ to 10d, respectively. The deprotection of the amino acid residues does not improve the cytotoxic activity but clearly improve the theoretical water solubility. The presence of dihydroxy groups on the phenyl substitution at position 1 appears to be necessary for the conservation of the cytotoxic activity.

Compounds 11a-11i are fully deprotected on the amino acid residues as well as on the catechol group at the position 1 . Among these compounds, five have attractive $\mathrm{IC}_{50}$ values below $1000 \mathrm{nM}$ : 403 , 584, 673, 838 and 951 for 11a, 11b, 11e, 11d and 11g, respectively. These compounds show ClogP values ranged from 2.57 to 4.32 for $\mathbf{1 1 f}$ and $\mathbf{1 1 d}$. Several compounds present very interesting theoretical water solubility: compounds 11a, 11b, 11c, 11d, 11g and 11h exhibit values higher than $3 \mathrm{mg} / \mathrm{mL}$. Very high values are obtained for compounds $\mathbf{1 1 a}$ and $\mathbf{1 1 b}$ at 47.18 and $19.92 \mathrm{mg} / \mathrm{mL}$, respectively. The presence of an alkyl or aryl moiety on the side chain of the amino acid residue does not decrease the water solubility for these compounds. Nevertheless, the tendency appears to not be the same for compounds 11e, 11f and 11i with theoretical water solubility values less than $1 \times 10^{-2} \mathrm{mg} / \mathrm{mL}$. These compounds possess ornithine (grafted by the $\alpha$-amine or by the amine of the side chain of the amino acid) or lysine residues. These two residues are therefore not valuable for increasing the water solubility of the compounds at $\mathrm{pH} 7.4$.

Compounds 11a, 11b, 11c and 11d present an alkyl chain at position 4 while compounds $\mathbf{1 1 g}$ and $\mathbf{1 1 h}$ display an aryl chain on this same position. Saturated alkyl groups appear to be most interesting for our compounds. A trend that seems to stand out in these four compounds is that when the carbon number of the alkyl chain increases, the biological activity and the theoretical solubility decrease (except for 11c which activity does not follow this tendency). Moreover, the presence of a phenyl group causes an increase in lipophilicity and a decrease in biological activities, in particular with the presence of a phenol (compound $\mathbf{1 1 h}$ ) on the side chain of the amino acid moiety. The compounds with lysine or ornithine residues did not show favorable results neither for biological activities or water solubility.

The transition of compounds $\mathbf{9}$ to compounds $\mathbf{1 1}$ is carried out by the cleavage of all the protecting groups of the amino acid moieties. Such a deprotection step forms less lipophilic compounds (lower $\mathrm{C} \log \mathrm{P}$ ) with similar or higher activity for all the amino acids tested, except for compounds $\mathbf{1 1 d}$ and $\mathbf{1 1 f}$ with the leucine and ornithine residues grafted by the amine in the $\alpha$-position, respectively. It should be noted that the presence of an ornithine amino acid residue grafted by the $\alpha$-amine or the amine of the side chain of the amino acid does not result in any significant differences in the biological activity (IC 50 values at $3404 \mathrm{nM}$ for $\mathbf{1 1 f}$ and $5168 \mathrm{nM}$ for 11i) or to the solubility (theoretical water solubility values at $5.82 \times 10^{-3}$ and $3.05 \times 10^{-3} \mathrm{mg} / \mathrm{mL}$ for $\mathbf{1 1 f}$ and 11i, respectively). Surprisingly, compound 11e which present a butan-1-amine substitution on the lateral chain of the amino acid show an higher biological activity than compound $\mathbf{1 1 f}$ with a propan-1-amine substitution on its lateral chain $\left(\mathrm{IC}_{50}\right.$ values at $673 \mathrm{nM}$ for 11e and $3404 \mathrm{nM}$ for 11f) with equivalent theoretical water solubilities (values of 4.34.10 -3 and $5.82 .10^{-3} \mathrm{mg} / \mathrm{mL}$ for $\mathbf{1 1 e}$ and $\mathbf{1 1 f}$, respectively). 
Consequently, compounds 11a, 11b, 11d and 11g in particular hold our attention, both in terms of improvement of the solubility and in terms of conservation of the biological activity. The activities of these compounds are in the same potency order as the leads of the first generation of imiqualines ( $\mathrm{IC}_{50}$ values of 200 and $1570 \mathrm{nM}$ for EAPB0503 and EAPB0203, respectively), presented in Table 3. Moreover, these new compounds show highly improved water solubility. Indeed, the values for the first generation compounds were only $2.60 \times 10^{-3}$ and $3.46 \times 10^{-3} \mathrm{mg} / \mathrm{mL}$ for EAPB0203 and EAPB0503, respectively. On the other hand, the second generation imiqualine compounds exhibit higher activities than the new compounds, with $\mathrm{IC}_{50}$ values at 10 and $60 \mathrm{nM}$ for EAPB02303 and EAPB02302 respectively. However, the solubility values of these compounds are low, with values of $1.74 \times 10^{-2} \mathrm{mg} / \mathrm{mL}$ for EAPB02303 and $4.28 \times 10^{-2} \mathrm{mg} / \mathrm{mL}$ for EAPB02302.

All of these data and results allow us to put forward a preferential amino acid with a small alkyl side chain in order to get a good compromise between maintaining the activity and increasing water solubility. From the close analysis of Tables 1 and 2, it can be observed that compounds 11a, 11b, 9d, 11d, 11e and 11g are the most active members of this series against the tested melanoma A375 cell line ( $\mathrm{IC}_{50}$ values less than $1000 \mathrm{nM}$ ). Surprisingly, compound $9 \mathrm{~d}$ shows the lowest $\mathrm{IC}_{50}$ value among these new synthesized compounds. Among these six attractive compounds, only 11a, 11b, 11d, 11e and 11g show a marked and considerable improvement of the theoretical water solubility.

\section{Experimental Section}

\subsection{Chemistry}

\subsubsection{General Information}

All solvents and reagents were obtained from Sigma Aldrich Chemical Co. (Saint Louis, MO, USA), Iris Biotech GmbH (Marktredwitz, Germany), Alfa Aesar Co. (Karlsruhe, Germany), VWR (Radnor, PA, USA) and FluoroChem UK (Hadfield, UK) and used without further purification unless indicated otherwise. Silica gel chromatography was conducted with 230-400 mesh $60 \AA$ silica gel (Sigma Aldrich Chemical Co.). The progress of reaction was monitored by TLC exposure to UV light (254 nM and $366 \mathrm{nM}$ ). Thin layer chromatography plates (Kieselgel 60 F254) were purchased from Merck (Darmstadt, Germany). Microwave assisted organic syntheses were performed on a Biotage Initiator 2.0 microwave system (Uppsala, Sweden). ${ }^{1} \mathrm{H}(400 \mathrm{MHz})$ and ${ }^{13} \mathrm{C}-\mathrm{NMR}(100 \mathrm{MHz})$ spectra were obtained on a Brüker AC-400 spectrometer (Billerica, MA, USA). Chemical shifts are given as parts per million $(\mathrm{ppm})$ using residual dimethylsulfoxide signal for protons $\left(\delta_{\mathrm{DMSO}}=2.46 \mathrm{ppm}\right)$ and carbons $\left(\delta_{\mathrm{DMSO}}=40.00 \mathrm{ppm}\right)$. Coupling constants are reported in Hertz $(\mathrm{Hz})$. Spectral splitting partners are designed as follow: singlet $(\mathrm{s})$; doublet $(\mathrm{d})$; triplet $(\mathrm{t})$; quartet $(\mathrm{q})$; multiplet $(\mathrm{m})$. Mass spectral data were obtained on a Waters Micromass Q-Tof (Milford, MA, USA) spectrometer equipped with ESI source (Laboratoires de Mesures Physiques, Plateau technique de l'Institut des Biomolecules Max Mousseron, Université de Montpellier, Montpellier, France). Mass spectra were recorded in positive mode between 50 and $1500 \mathrm{Da}$, capillary and cone tension were 3000 and $20 \mathrm{~V}$, respectively. The High Resolution Mass Spectroscopy (HRMS) analyses are carried out by direct introduction on a Synapt G2-S mass spectrometer (Waters, SN: UEB205) equipped with ESI source. The mass spectra were recorded in positive mode, between 100 and $1500 \mathrm{Da}$. The capillary tension is $1000 \mathrm{~V}$ and the cone tension is $30 \mathrm{~V}$. The source and desolvation temperature are $120^{\circ} \mathrm{C}$ and $250{ }^{\circ} \mathrm{C}$, respectively. NMR ${ }^{1} \mathrm{H}$ and ${ }^{13} \mathrm{C}$ spectra of all compounds are in Supplementary Materials.

\subsubsection{Amino Acids Grafted on 4-Chloroimidazo[1,2-a]quinoxaline}

Tert-butyl 2-(imidazo[1,2-a]quinoxalin-4-ylamino)acetate (6a): Glycine tert-butyl ester hydrochloride (0.824 g, $4.9 \mathrm{mmol}), \mathrm{N}, \mathrm{N}$-diisopropylethylamine $(1.6 \mathrm{~mL}, 9.8 \mathrm{mmol})$ and 4-chloroimidazo[1,2-a]quinoxaline $5(0.5 \mathrm{~g}$, $2.5 \mathrm{mmol}$ ) were dissolved in dimethyl-formamide $(10 \mathrm{~mL})$ in a microwave adapted vial and sealed. The reaction mixture was irradiated at $150{ }^{\circ} \mathrm{C}$ for $30 \mathrm{~min}$. The solvent was removed under reduced 
pressure. The crude mixture was dissolved in ethyl acetate $(100 \mathrm{~mL})$ and successively washed with saturated aqueous ammonium chloride, distilled water and finally brine. The organic phase was dried on sodium sulfate, filtered and concentrated under reduced pressure. The product was purified by flash chromatography eluted with cyclohexane/ethyl acetate $80 / 20$ to $50 / 50$. The compound is obtained as a beige solid (42\% yield). $\mathrm{C}_{16} \mathrm{H}_{18} \mathrm{~N}_{4} \mathrm{O}_{2} . \mathrm{M}_{\mathrm{W}}: 298.34 \mathrm{~g} / \mathrm{mol} .{ }^{1} \mathrm{H}-\mathrm{NMR} \delta\left(\mathrm{ppm}, \mathrm{DMSO}-d_{6}\right): 1.42(\mathrm{~s}, 9 \mathrm{H}$, $\left.3 \times \mathrm{CH}_{3} \mathrm{OtBu}\right), 4.13\left(\mathrm{~d}, 2 \mathrm{H}, J=8 \mathrm{~Hz}, \mathrm{CH}_{2} \alpha\right), 7.31-7.35(\mathrm{~m}, 1 \mathrm{H}, \mathrm{CH}$ 7), 7.40-7.44 (m, 1H, CH 8), 7.56 (dd, $1 \mathrm{H}, J=4 \mathrm{~Hz}, J=8 \mathrm{~Hz}, \mathrm{CH} 9), 7.66(\mathrm{~d}, 1 \mathrm{H}, J=4 \mathrm{~Hz}, \mathrm{CH} 2), 7.96(\mathrm{t}, 1 \mathrm{H}, J=8 \mathrm{~Hz}, \mathrm{NH}), 8.13(\mathrm{dd}, 1 \mathrm{H}, J=4 \mathrm{~Hz}$, $J=8 \mathrm{~Hz}, \mathrm{CH}$ 6), $8.64(\mathrm{~d}, 1 \mathrm{H}, J=4 \mathrm{~Hz}, \mathrm{CH} 1) .{ }^{13} \mathrm{C}-\mathrm{NMR} \delta\left(\mathrm{ppm}, \mathrm{DMSO}-d_{6}\right): 28.23\left(\mathrm{CH}_{3} \mathrm{tBu}\right), 43.30\left(\mathrm{CH}_{2}\right.$ $\alpha), 80.85(\mathrm{Cq} \mathrm{tBu}), 115.14(\mathrm{CH}$ ) $), 115.91(\mathrm{CH} 6), 123.57(\mathrm{CH} \mathrm{7}), 124.93$ (Cq 5a), $126.74(\mathrm{CH} 9), 126.88(\mathrm{CH}$ 8), 132.52 (CH 2), 132.71 (Cq 3a), 136.81 (Cq 9a), 147.49 (Cq 4), 169.79 (C=O). MS (ESI +, QTof, m/z): 299.0 $[\mathrm{M}+\mathrm{H}]^{+}$.

Tert-butyl 2-(imidazo[1,2-a]quinoxalin-4-ylamino)propanoate (6b): Same procedure used for the synthesis of $6 \mathbf{a}$ was employed. L-Alanine tert-butyl ester hydrochloride (1.339 $\mathrm{g}, 7.4 \mathrm{mmol})$, $\mathrm{N}, \mathrm{N}$-diisopropylethylamine $(2.4 \mathrm{~mL}, 14.7 \mathrm{mmol})$ and 4-chloroimidazo[1,2-a]quinoxaline 5 (0.5 g, $2.5 \mathrm{mmol})$ were dissolved and reacted in dimethyl-formamide $(10 \mathrm{~mL})$. The product was purified by flash chromatography eluted with cyclohexane/ethyl acetate $80 / 20$ to $50 / 50$. The compound is obtained as a beige solid (48\% yield). $\mathrm{C}_{17} \mathrm{H}_{20} \mathrm{~N}_{4} \mathrm{O}_{2} . \mathrm{M}_{\mathrm{W}}: 312.37 \mathrm{~g} / \mathrm{mol} .{ }^{1} \mathrm{H}-\mathrm{NMR} \delta$ (ppm, DMSO- $d_{6}$ ): $1.41\left(\mathrm{~s}, 9 \mathrm{H}, 3 \times \mathrm{CH}_{3} \mathrm{OtBu}\right), 1.51\left(\mathrm{~d}, 3 \mathrm{H}, J=8 \mathrm{~Hz}, \mathrm{CH}_{3} \beta\right), 4.56-4.63(\mathrm{~m}, 1 \mathrm{H}, \mathrm{CH} \alpha), 7.33-7.36(\mathrm{~m}, 1 \mathrm{H}$, CH 7), 7.41-7.43 (m, 1H, CH 8), $7.56(\mathrm{dd}, 1 \mathrm{H}, J=4 \mathrm{~Hz}, J=8 \mathrm{~Hz}, \mathrm{CH} 9), 7.66(\mathrm{~d}, 1 \mathrm{H}, J=4 \mathrm{~Hz}, \mathrm{CH}$ 2), 7.75 $(\mathrm{d}, 1 \mathrm{H}, J=4 \mathrm{~Hz}, \mathrm{NH}), 8.13(\mathrm{dd}, 1 \mathrm{H}, J=4 \mathrm{~Hz}, J=8 \mathrm{~Hz}, \mathrm{CH} 6), 8.64(\mathrm{~d}, 1 \mathrm{H}, J=4 \mathrm{~Hz}, \mathrm{CH} 1) .{ }^{13} \mathrm{C}-\mathrm{NMR} \delta$ (ppm, DMSO- $\left.d_{6}\right)$ : $17.46\left(\mathrm{CH}_{3} \beta\right), 28.14\left(\mathrm{CH}_{3} \mathrm{tBu}\right), 50.22(\mathrm{CH} \alpha), 80.59(\mathrm{Cq} \mathrm{tBu}), 115.18(\mathrm{CH} 1), 115.91$ (CH 6), 123.64 (CH 7), 124.93 (Cq 5a), 126.73 (CH 9), 126.88 (CH 8), 132.42 (CH 2), 132.58 (Cq 3a), 136.72 (Cq 9a), 147.03 (Cq 4), $172.84(\mathrm{C}=\mathrm{O})$. MS (ESI +, QTof, $m / z)$ : $313.2[\mathrm{M}+\mathrm{H}]^{+}$.

Tert-Butyl 2-(imidazo[1,2-a]quinoxalin-10-ylamino)-3-methylbutanoate (6c): Using the same procedure as for the synthesis of 6a, L-valine tert-butyl ester hydrochloride $(2.060 \mathrm{~g}, 9.8 \mathrm{mmol})$, $\mathrm{N}, \mathrm{N}$-diisopropylethylamine $(2.4 \mathrm{~mL}, 14.7 \mathrm{mmol})$ and 4-chloroimidazo[1,2-a]quinoxaline $5(0.5 \mathrm{~g}$, $2.5 \mathrm{mmol})$ were mixed in dimethylformamide $(10 \mathrm{~mL})$. The product was purified by flash chromatography eluted with cyclohexane/ethyl acetate $80 / 20$ to $50 / 50$. The compound is obtained as a beige solid (51\% yield). $\mathrm{C}_{19} \mathrm{H}_{24} \mathrm{~N}_{4} \mathrm{O}_{2} . \mathrm{M}_{\mathrm{W}}: 340.42 \mathrm{~g} / \mathrm{mol} .{ }^{1} \mathrm{H}-\mathrm{NMR} \delta$ (ppm, DMSO- $d_{6}$ ): $1.03(\mathrm{~d}, 3 \mathrm{H}$, $\left.J=8 \mathrm{~Hz}, \mathrm{CH}_{3} \gamma\right), 1.06\left(\mathrm{~d}, 3 \mathrm{H}, J=8 \mathrm{~Hz}, \mathrm{CH}_{3} \gamma^{\prime}\right), 1.43\left(\mathrm{~s}, 9 \mathrm{H}, 3 \times \mathrm{CH}_{3} \mathrm{OtBu}\right), 2.29-2.37(\mathrm{~m}, 1 \mathrm{H}, \mathrm{CH} \beta)$, $4.52(\mathrm{t}, 1 \mathrm{H}, J=16 \mathrm{~Hz}, \mathrm{CH} \alpha), 7.15(\mathrm{~d}, 1 \mathrm{H}, J=8 \mathrm{~Hz}, \mathrm{NH}), 7.33-7.37(\mathrm{~m}, 1 \mathrm{H}, \mathrm{CH} 7), 7.42-7.44(\mathrm{~m}, 1 \mathrm{H}, \mathrm{CH}$ 8), $7.59(\mathrm{dd}, 1 \mathrm{H} \mathrm{J}=4 \mathrm{~Hz}, J=8 \mathrm{~Hz}, \mathrm{CH} 9), 7.68(\mathrm{~d}, 1 \mathrm{H}, J=4 \mathrm{~Hz}, \mathrm{CH} 2), 8.14(\mathrm{dd}, 1 \mathrm{H}, J=4 \mathrm{~Hz}, J=8 \mathrm{~Hz}$, $\mathrm{CH}$ 6), $8.66(\mathrm{~d}, 1 \mathrm{H}, J=4 \mathrm{~Hz}, \mathrm{CH} 1) .{ }^{13} \mathrm{C}-\mathrm{NMR} \delta\left(\mathrm{ppm}, \mathrm{DMSO}-d_{6}\right): 19.42\left(\mathrm{CH}_{3} \gamma, \mathrm{CH}_{3} \gamma^{\prime}\right), 28.17\left(\mathrm{CH}_{3}\right.$ $\mathrm{tBu}), 30.41(\mathrm{CH} \beta), 59.76(\mathrm{CH} \alpha), 81.15(\mathrm{Cq} \mathrm{tBu}), 115.37(\mathrm{CH} \mathrm{1}), 115.95(\mathrm{CH} 6), 123.88(\mathrm{CH} 7), 125.00(\mathrm{Cq}$ 5a), 126.84 (CH 9), 126.95 (CH 8), 132.48 (CH 2), 132.58 (Cq 3a), 136.59 (Cq 9a), 147.29 (Cq 4), 171.59 $(\mathrm{C}=\mathrm{O})$. MS (ESI +, QTof, $m / z): 341.0[\mathrm{M}+\mathrm{H}]^{+}$.

Tert-butyl 2-(imidazo[1,2-a]quinoxalin-4-ylamino)-4-methylpentanoate (6d): The same as for the synthesis of 6a was used, employing L-leucine tert-butyl ester hydrochloride (2.198 g, $9.8 \mathrm{mmol})$, $\mathrm{N}, \mathrm{N}$-diisopropylethylamine $(2.4 \mathrm{~mL}, 14.7 \mathrm{mmol})$ and 4-chloro-imidazo[1,2-a]quinoxaline $5(0.5 \mathrm{~g}$, $2.5 \mathrm{mmol}$ ) in dimethylformamide $(10 \mathrm{~mL})$. The product was purified by flash chromatography eluted with cyclohexane/ethyl acetate $80 / 20$ to $50 / 50$. The compound is obtained as a beige solid (60\% yield). $\mathrm{C}_{20} \mathrm{H}_{26} \mathrm{~N}_{4} \mathrm{O}_{2} . \mathrm{M}_{\mathrm{W}}: 354.45 \mathrm{~g} / \mathrm{mol} .{ }^{1} \mathrm{H}-\mathrm{NMR} \delta$ (ppm, DMSO- $\left.d_{6}\right): 0.93\left(\mathrm{~d}, 3 \mathrm{H}, J=8 \mathrm{~Hz}, \mathrm{CH}_{3} \delta\right), 0.96(\mathrm{~d}$, $\left.3 \mathrm{H}, J=8 \mathrm{~Hz}, \mathrm{CH}_{3} \delta^{\prime}\right), 1.40\left(\mathrm{~s}, 9 \mathrm{H}, 3 \times \mathrm{CH}_{3} \mathrm{OtBu}\right), 1.60-1.67\left(\mathrm{~m}, 1 \mathrm{H}, \mathrm{CH}_{2} \beta\right), 1.77-1.79(\mathrm{~m}, 1 \mathrm{H}, \mathrm{CH} \gamma)$, 1.93-1.95 (m, 1H, CH $\beta$ ß $), 4.63(\mathrm{t}, 1 \mathrm{H}, J=4 \mathrm{~Hz}, \mathrm{CH} \alpha), 7.26-7.33(\mathrm{~m}, 1 \mathrm{H}, \mathrm{CH} 7), 7.40-7.44(\mathrm{~m}, 1 \mathrm{H}, \mathrm{CH} 8)$, $7.57(\mathrm{dd}, 1 \mathrm{H}, J=4 \mathrm{~Hz}, J=8 \mathrm{~Hz}, \mathrm{CH} 9), 7.64(\mathrm{~d}, 1 \mathrm{H}, J=4 \mathrm{~Hz}, \mathrm{NH}), 7.66(\mathrm{~d}, 1 \mathrm{H}, J=4 \mathrm{~Hz}, \mathrm{CH} 2), 8.13(\mathrm{dd}$, $1 \mathrm{H}, J=4 \mathrm{~Hz}, J=8 \mathrm{~Hz}, \mathrm{CH} 6), 8.64(\mathrm{~d}, 1 \mathrm{H}, J=4 \mathrm{~Hz}, \mathrm{CH} 1) .{ }^{13} \mathrm{C}-\mathrm{NMR} \delta\left(\mathrm{ppm}, \mathrm{DMSO}-d_{6}\right): 22.06\left(\mathrm{CH}_{3} \delta\right)$, $23.31\left(\mathrm{CH}_{3} \delta^{\prime}\right), 25.14(\mathrm{CH} \gamma), 28.15\left(\mathrm{CH}_{3} \mathrm{tBu}\right), 40.01\left(\mathrm{CH}_{2} \beta\right), 52.93(\mathrm{CH} \alpha), 80.67(\mathrm{Cq} \mathrm{tBu}), 115.21(\mathrm{CH}$ 1), 115.90 (CH 6), 123.64 (CH 7), 124.93 (Cq 5a), $126.76(\mathrm{CH} \mathrm{8),} 126.89$ (CH 9), $132.41(\mathrm{CH} 2), 132.58$ (Cq 3a), 136.74 (Cq 9a), 147.40 (Cq 4), 172.75 (C=O). MS (ESI +, QTof, $m / z): 355.0[\mathrm{M}+\mathrm{H}]^{+}$. 
Tert-butyl 6-((tert-butoxycarbonyl)amino)-2-(imidazo[1,2-a]quinoxalin-4-ylamino)hexanoate (6e): The same procedure used for the synthesis of 6 a was employed with $N$ - $\varepsilon$-tert-butyloxycarbonyl-L-lysine tert-butyl ester hydrochloride ( $2.565 \mathrm{~g}, 7.6 \mathrm{mmol}), \mathrm{N}, \mathrm{N}$-diisopropyl-ethylamine $(2.4 \mathrm{~mL}, 14.7 \mathrm{mmol})$ and 4-chloroimidazo[1,2-a]quinoxaline $5(0.5 \mathrm{~g}, 2.5 \mathrm{mmol})$ in dimethylformamide $(10 \mathrm{~mL})$. The product was purified by flash chromatography eluted with cyclohexane/ethyl acetate $80 / 20$ to $50 / 50$. The compound is obtained as a beige solid (32\% yield). $\mathrm{C}_{25} \mathrm{H}_{35} \mathrm{~N}_{5} \mathrm{O}_{4} . \mathrm{M}_{\mathrm{W}}: 469.58 \mathrm{~g} / \mathrm{mol} .{ }^{1} \mathrm{H}-\mathrm{NMR} \delta$ (ppm, DMSO- $\left.d_{6}\right): 1.35\left(\mathrm{~s}, 9 \mathrm{H}, 3 \times \mathrm{CH}_{3} \mathrm{OtBu}\right), 1.41\left(\mathrm{~s}, 9 \mathrm{H}, 3 \times \mathrm{CH}_{3} \mathrm{OtBu}\right), 1.43-1.49\left(\mathrm{~m}, 4 \mathrm{H}, \mathrm{CH}_{2} \gamma, \mathrm{CH}_{2}\right.$ ঠ), 1.86-1.92 (m, 2H, $\left.\mathrm{CH}_{2} \beta\right), 2.91-2.93\left(\mathrm{~m}, 2 \mathrm{H}, \mathrm{CH}_{2} \varepsilon\right), 4.52-4.56(\mathrm{~m}, 1 \mathrm{H}, \mathrm{CH} \alpha), 6.79(\mathrm{t}, 1 \mathrm{H}, J=4 \mathrm{~Hz}$, $\left.\mathrm{NH}-\mathrm{CH}_{2} \varepsilon\right), 7.26-7.35(\mathrm{~m}, 1 \mathrm{H}, \mathrm{CH} 7), 7.39-7.42(\mathrm{~m}, 1 \mathrm{H}, \mathrm{CH} 8), 7.52(\mathrm{dd}, 1 \mathrm{H}, J=4 \mathrm{~Hz}, J=8 \mathrm{~Hz}, \mathrm{CH} 9)$, $7.64(\mathrm{~d}, 1 \mathrm{H}, J=4 \mathrm{~Hz}, \mathrm{NH}-\mathrm{CH} \alpha), 7.66(\mathrm{~d}, 1 \mathrm{H}, J=4 \mathrm{~Hz}, \mathrm{CH} 2), 8.12(\mathrm{dd}, 1 \mathrm{H}, J=4 \mathrm{~Hz}, J=8 \mathrm{~Hz}, \mathrm{CH} 6)$, $8.64(\mathrm{~d}, 1 \mathrm{H}, J=4 \mathrm{~Hz}, \mathrm{CH} 1) .{ }^{13} \mathrm{C}-\mathrm{NMR} \delta\left(\mathrm{ppm}, \mathrm{DMSO}-d_{6}\right): 23.52\left(\mathrm{CH}_{2} \gamma\right), 28.19\left(\mathrm{CH}_{3} \mathrm{tBu}\right), 28.74\left(\mathrm{CH}_{3}\right.$ $\mathrm{tBu}), 29.67\left(\mathrm{CH}_{2} \delta\right), 30.92\left(\mathrm{CH}_{2} \beta\right), 40.35\left(\mathrm{CH}_{2} \varepsilon\right), 54.64(\mathrm{CH} \alpha), 79.68(\mathrm{Cq} \mathrm{tBu}), 80.80(\mathrm{Cq} \mathrm{tBu}), 115.25$ (CH 1), 115.93 (CH 6), 123.70 (CH 7), 124.98 (Cq 5a), 126.81 (CH 9), 126.92 (CH 8), 132.44 (CH 2), 132.60 (Cq 3a), 136.75 (Cq 9a), 147.35 (Cq 4), 172.41 (C=O). MS (ESI +, QTof, m/z): $470.0[\mathrm{M}+\mathrm{H}]^{+}$.

Tert-butyl 5-(((benzyloxy)carbonyl)amino)-2-(imidazo[1,2-a]quinoxalin-4-ylamino)-pentanoate (6f): Using the same procedure used for the synthesis of 6 a with $\mathrm{N}-\delta$-carbobenzoxy-L-ornithine $\alpha$-tert-butyl ester hydrochloride $(3.702 \mathrm{~g}, 10.3 \mathrm{mmol}), \mathrm{N}, \mathrm{N}$-diisopropylethylamine $(2.4 \mathrm{~mL}, 14.7 \mathrm{mmol})$ and 4-chloroimidazo[1,2-a]quinoxaline $5(0.5 \mathrm{~g}, 2.5 \mathrm{mmol})$ in dimethylformamide $(10 \mathrm{~mL})$. The product was purified by flash chromatography eluted with cyclohexane/ethyl acetate $80 / 20$ to $50 / 50$. The compound is obtained as a beige solid ( $38 \%$ yield). $\mathrm{C}_{27} \mathrm{H}_{31} \mathrm{~N}_{5} \mathrm{O}_{4} \cdot \mathrm{M}_{\mathrm{W}}: 489.57 \mathrm{~g} / \mathrm{mol} .{ }^{1} \mathrm{H}-\mathrm{NMR} \delta$ (ppm, $400 \mathrm{MHz}$, DMSO- $\left.d_{6}\right):{ }^{1} \mathrm{H}-\mathrm{NMR} \delta$ (ppm, DMSO- $\left.d_{6}\right): 1.29$ (s, 9H, $\left.3 \times \mathrm{CH}_{3} \mathrm{OtBu}\right), 1.46-1.50(\mathrm{~m}$, $\left.2 \mathrm{H}, \mathrm{CH}_{2} \gamma\right), 1.79-1.84\left(\mathrm{~m}, 2 \mathrm{H}, \mathrm{CH}_{2} \beta\right), 2.94-2.98\left(\mathrm{~m}, 2 \mathrm{H}, \mathrm{CH}_{2} \delta\right), 4.44-4.46(\mathrm{~m}, 1 \mathrm{H}, \mathrm{CH} \alpha), 4.90(\mathrm{~s}, 2 \mathrm{H}$, $\left.\mathrm{CH}_{2}-\mathrm{Phenyl}\right), 7.17-7.21$ ( $\mathrm{m}, 1 \mathrm{H}, \mathrm{CH}$ 7), 7.23 (s, 1H, NH-CH ${ }_{2}$ ), $7.24-7.27$ (m, 5H, CH Phenyl), 7.29-7.33 $(\mathrm{m}, 1 \mathrm{H}, \mathrm{CH} 8), 7.46(\mathrm{dd}, 1 \mathrm{H}, J=4 \mathrm{~Hz}, J=8 \mathrm{~Hz}, \mathrm{CH} 9), 7.52(\mathrm{~d}, 1 \mathrm{H}, J=4 \mathrm{~Hz}, \mathrm{NH}-\mathrm{CH} \alpha), 7.56(\mathrm{~d}, 1 \mathrm{H}$, $J=4 \mathrm{~Hz}, \mathrm{CH} 2), 8.02(\mathrm{dd}, 1 \mathrm{H}, J=4 \mathrm{~Hz}, J=8 \mathrm{~Hz}, \mathrm{CH} 6), 8.53(\mathrm{~d}, 1 \mathrm{H}, J=4 \mathrm{~Hz}, \mathrm{CH} 1) .{ }^{13} \mathrm{C}-\mathrm{NMR} \delta$ (ppm, DMSO-d $\left.d_{6}\right): 26.63\left(\mathrm{CH}_{2} \gamma\right), 28.13\left(\mathrm{CH}_{3} \mathrm{tBu}\right), 28.66\left(\mathrm{CH}_{2} \beta\right), 40.71\left(\mathrm{CH}_{2} \delta\right), 54.50(\mathrm{CH} \alpha), 65.67$ ( $\mathrm{CH}_{2}$-Phenyl), 80.91 (Cq tBu), 115.30 (CH 1), 115.99 (CH 6), 123.77 (CH 7), 125.03 (Cq 5a), 126.84 (CH 9), 126.97 (CH 8), 128.86 (CH Phenyl), 132.50 (CH 2), 132.65 (Cq 3a), 136.77 (Cq 9a), 147.36 (Cq 4), 156.65 (Cq Phenyl), 172.28 (C=O). MS (ESI +, QTof, $m / z): 490.0[\mathrm{M}+\mathrm{H}]^{+}$.

Tert-Butyl 2-(imidazo[1,2-a]quinoxalin-4-ylamino)-3-phenylpropanoate (6g):The same procedure as for the synthesis of $\mathbf{6 a}$ was used with L-phenylalanine tert-butyl ester hydrochloride $(1.9 \mathrm{~g}, 7.4 \mathrm{mmol})$, $\mathrm{N}, \mathrm{N}$-diisopropylethylamine $(2.4 \mathrm{~mL}, 14.7 \mathrm{mmol})$ and 4-chloroimidazo[1,2-a]quinoxaline $5(0.5 \mathrm{~g}$, $2.5 \mathrm{mmol})$ in dimethylformamide $(10 \mathrm{~mL})$. The product was purified by flash chromatography eluted with cyclohexane/ethyl acetate $80 / 20$ to $50 / 50$. The compound is obtained as a beige solid (37\% yield). $\mathrm{C}_{23} \mathrm{H}_{24} \mathrm{~N}_{4} \mathrm{O}_{2} . \mathrm{M}_{\mathrm{W}}: 388.46 \mathrm{~g} / \mathrm{mol} .{ }^{1} \mathrm{H}-\mathrm{NMR} \delta$ (ppm, DMSO-d $\left.d_{6}\right): 1.33$ (s, 9H, $3 \times \mathrm{CH}_{3} \mathrm{OtBu}$ ), 3.19-3.23 $\left(\mathrm{m}, 1 \mathrm{H}, \mathrm{CH}_{2} \beta\right), 3.32-3.36\left(\mathrm{~m}, 1 \mathrm{H}, \mathrm{CH}_{2} \beta\right), 4.82-4.84(\mathrm{~m}, 1 \mathrm{H}, \mathrm{CH} \alpha), 7.21(\mathrm{t}, 1 \mathrm{H}, J=8 \mathrm{~Hz}, \mathrm{CH}$ Phenyl), $7.29(\mathrm{t}, 2 \mathrm{H}, J=8 \mathrm{~Hz}, 2 \times \mathrm{CH}$ Phenyl), 7.32-7.34 (m, 3H, CH 7, $2 \times \mathrm{CH}$ Phenyl), 7.41-7.44 (m, 1H, CH 8), $7.57(\mathrm{dd}, 1 \mathrm{H}, J=4 \mathrm{~Hz}, J=8 \mathrm{~Hz}, \mathrm{CH} 9), 7.61(\mathrm{~d}, 1 \mathrm{H}, J=8 \mathrm{~Hz}, \mathrm{NH}), 7.66(\mathrm{~d}, 1 \mathrm{H}, J=4 \mathrm{~Hz}, \mathrm{CH} 2), 8.13$ $(\mathrm{dd}, 1 \mathrm{H}, J=8,4 \mathrm{~Hz}, \mathrm{CH} 6), 8.64(\mathrm{~d}, 1 \mathrm{H}, J=4 \mathrm{~Hz}, \mathrm{CH} 1) .{ }^{13} \mathrm{C}-\mathrm{NMR} \delta\left(\mathrm{ppm}, \mathrm{DMSO}-\mathrm{d}_{6}\right): 26.97\left(\mathrm{CH}_{3} \mathrm{tBu}\right)$, $35.91\left(\mathrm{CH}_{2} \beta\right), 54.93(\mathrm{CH} \alpha), 79.88(\mathrm{Cq} \mathrm{tBu}), 114.18(\mathrm{CH} 1), 114.85(\mathrm{CH} 6), 122.74(\mathrm{CH} 7), 123.89(\mathrm{Cq} 5 \mathrm{a})$, 125.74 (CH 9), 125.84 (CH Phenyl), 125.86 (CH 8), 127.59 (CH Phenyl), 128.69 (CH Phenyl), 131.41 (CH 2), 135.54 (Cq 3a), 137.05 (Cq 9a), 146.02 (Cq 4), 170.53 (C=O). MS (ESI +, QTof, $m / z): 389.0[\mathrm{M}+\mathrm{H}]^{+}$.

Tert-butyl 3-(4-(tert-butoxy)phenyl)-2-(imidazo[1,2-a]quinoxalin-4-ylamino)propanoate (6h): The same procedure as for the synthesis of $\mathbf{6 a}$ was used with O-tert-butyl-L-tyrosine tert-butyl ester hydrochloride (1.6 g, $4.9 \mathrm{mmol}), \mathrm{N}, \mathrm{N}$-diisopropylethylamine $(2.4 \mathrm{~mL}, 14.7 \mathrm{mmol})$ and 4-chloroimidazo[1,2-a]quinoxaline $5(0.5 \mathrm{~g}, 2.5 \mathrm{mmol})$ in dimethylformamide $(10 \mathrm{~mL})$. The product was purified by flash chromatography eluted with cyclohexane/ethyl acetate $80 / 20$ to $50 / 50$. The compound is obtained as a beige solid ( $26 \%$ yield). $\mathrm{C}_{27} \mathrm{H}_{32} \mathrm{~N}_{4} \mathrm{O}_{3} . \mathrm{M}_{\mathrm{W}}: 460.57 \mathrm{~g} / \mathrm{mol} .{ }^{1} \mathrm{H}-\mathrm{NMR} \delta(\mathrm{ppm}$, DMSO- $\left.d_{6}\right): 1.23\left(\mathrm{~s}, 9 \mathrm{H}, 3 \times \mathrm{CH}_{3} \mathrm{OtBu}\right), 1.31\left(\mathrm{~s}, 9 \mathrm{H}, 3 \times \mathrm{CH}_{3} \mathrm{OtBu}\right), 3.11-3.17\left(\mathrm{~m}, 1 \mathrm{H}, \mathrm{CH}_{2} \beta\right), 3.25-3.31$ 
$\left(\mathrm{m}, 1 \mathrm{H}, \mathrm{CH}_{2} \beta\right), 4.78-4.83(\mathrm{~m}, 1 \mathrm{H}, \mathrm{CH} \alpha), 6.86(\mathrm{dd}, 2 \mathrm{H}, J=4 \mathrm{~Hz}, J=8 \mathrm{~Hz}, \mathrm{CH}$ Phenyl), $7.22(\mathrm{dd}, 2 \mathrm{H}$, $J=4 \mathrm{~Hz}, J=8 \mathrm{~Hz}, \mathrm{CH}$ Phenyl), 7.32-7.36 (m, 1H, CH 7), 7.40-7.44 (m, 1H, CH 8), 7.57 (dd, 1H, J = $4 \mathrm{~Hz}$, $J=8 \mathrm{~Hz}, \mathrm{CH}$ 9), $7.61(\mathrm{~d}, 1 \mathrm{H}, J=4 \mathrm{~Hz}, \mathrm{NH}), 7.66(\mathrm{~d}, 1 \mathrm{H}, J=4 \mathrm{~Hz}, \mathrm{CH} 2), 8.13(\mathrm{dd}, 1 \mathrm{H}, J=4 \mathrm{~Hz}, J=8 \mathrm{~Hz}$, $\mathrm{CH}$ 6), $8.64(\mathrm{~d}, 1 \mathrm{H}, J=4 \mathrm{~Hz}, \mathrm{CH} 1) .{ }^{13} \mathrm{C}-\mathrm{NMR} \delta$ (ppm, DMSO- $\left.d_{6}\right): 28.02\left(\mathrm{CH}_{3} \mathrm{tBu}\right), 28.93\left(\mathrm{CH}_{3} \mathrm{tBu}\right)$, $36.63\left(\mathrm{CH}_{2} \beta\right), 56.09(\mathrm{CH} \alpha), 78.11(\mathrm{Cq} \mathrm{tBu}), 80.87(\mathrm{Cq} \mathrm{tBu}), 115.22(\mathrm{CH} \mathrm{1}), 115.90(\mathrm{CH} 6), 123.80(\mathrm{CH} 7)$, 123.98 ( $2 \times$ CH Phenyl), 124.96 (Cq 5a), 126.79 (CH 9), 126.90 (CH 8), 130.31 (2 × CH Phenyl), 132.47 (CH 2), 132.61 (Cq 3a), 136.60 (Cq 9a), 147.04 (Cq 4), 154.03 (2 × Cq Phenyl), 171.72 (C=O). MS (ESI +, QTof, $m / z): 461.2[\mathrm{M}+\mathrm{H}]^{+}$.

Tert-butyl 6-((tert-butoxycarbonyl)amino)-2-(imidazo[1,2-a]quinoxalin-4-ylamino)hexanoate (6i): Same procedure used for the synthesis of $\mathbf{6 a}$ was employed with $N$ - $\alpha$-tert-butyloxycarbonyl-L-ornithine tert-butyl ester hydrochloride $(1.596 \mathrm{~g}, 4.9 \mathrm{mmol}), \mathrm{N}, \mathrm{N}$-diisopropyl-ethylamine $(1.6 \mathrm{~mL}, 9.8 \mathrm{mmol})$ and 4-chloroimidazo[1,2-a]quinoxaline $5(0.5 \mathrm{~g}, 2.5 \mathrm{mmol})$ in dimethylformamide $(10 \mathrm{~mL})$. The product was purified by flash chromatography eluted with cyclohexane/ethyl acetate $80 / 20$ to $50 / 50$. The compound is obtained as a beige solid (48\% yield). $\mathrm{C}_{24} \mathrm{H}_{33} \mathrm{~N}_{5} \mathrm{O}_{4} . \mathrm{M}_{\mathrm{W}}: 455.56 \mathrm{~g} / \mathrm{mol} .{ }^{1} \mathrm{H}-\mathrm{NMR} \delta$ (ppm, DMSO- $\left.d_{6}\right): 1.35$ (s, 9H, CH-COOtBu), 1.41 (s, 9H, NH-COOtBu), 1.85-1.96 (m, 2H, $\left.\mathrm{CH}_{2} \beta\right), 2.91-2.95$ $\left(\mathrm{m}, 2 \mathrm{H}, \mathrm{CH}_{2} \gamma\right), 3.35-3.39\left(\mathrm{~m}, 2 \mathrm{H}, \mathrm{CH}_{2} \delta\right), 4.51-4.56(\mathrm{~m}, 1 \mathrm{H}, \mathrm{CH} \alpha), 6.79(\mathrm{~d}, 1 \mathrm{H}, J=4 \mathrm{~Hz}, \mathrm{NH}-\mathrm{CH} \alpha)$, 7.32-7.35 (m, 1H, CH 7), 7.39-7.42 (m, 1H, CH 8), $7.56(\mathrm{dd}, 1 \mathrm{H}, J=4 \mathrm{~Hz}, J=8 \mathrm{~Hz}, \mathrm{CH} 9), 7.60(\mathrm{~d}, 1 \mathrm{H}$, $\left.J=4 \mathrm{~Hz}, \mathrm{NH}-\mathrm{CH}_{2} \delta\right), 7.66(\mathrm{~s}, 1 \mathrm{H}, \mathrm{CH} 2), 8.13(\mathrm{dd}, 1 \mathrm{H}, J=4 \mathrm{~Hz}, J=8 \mathrm{~Hz}, \mathrm{CH} 6), 8.64(\mathrm{~d}, 1 \mathrm{H}, \mathrm{CH} 1)$. ${ }^{13} \mathrm{C}-\mathrm{NMR} \delta$ (ppm, DMSO- $\left.d_{6}\right): 25.87\left(\mathrm{CH}_{2} \beta\right), 28.04\left(\mathrm{CH}_{3} \mathrm{tBu}\right), 28.67\left(\mathrm{CH}_{3} \mathrm{tBu}\right), 28.43\left(\mathrm{CH}_{2} \gamma\right), 39.79$ $\left(\mathrm{CH}_{2} \delta\right), 54.75(\mathrm{CH} \alpha), 78.45(\mathrm{Cq} \mathrm{tBu}), 80.53(\mathrm{Cq} \mathrm{tBu}), 115.02(\mathrm{CH} 1), 115.79(\mathrm{CH} 6), 122.99(\mathrm{CH} 7), 124.65$ (Cq 5a), 126.50 (CH 9), 126.73 (CH 8), 132.20 (CH 2), 132.87 (Cq 3a), 147.78 (Cq 4), 156.00 (Cq 9a), 172.33 $(\mathrm{C}=\mathrm{O})$. MS (ESI +, QTof, $m / z): 456.0 .1[\mathrm{M}+\mathrm{H}]^{+}$.

\subsubsection{Bromination}

Tert-butyl 2-((1-bromoimidazo[1,2-a]quinoxalin-4-yl)amino)acetate (7a): A solution of $6 \mathbf{a}(0.26 \mathrm{~g}, 0.87 \mathrm{mmol})$ and $\mathrm{N}$-bromosuccinimide $(0.19 \mathrm{~g}, 1.0 \mathrm{mmol})$ in $\mathrm{CHCl}_{3}(50 \mathrm{~mL})$ was heated under reflux for $2 \mathrm{~h}$. The solvent was removed under reduced pressure. The crude mixture was dissolved in ethyl acetate $(50 \mathrm{~mL})$ and successively washed with saturated aqueous ammonium chloride, saturated aqueous sodium bicarbonate, distilled water and finally brine $(50 \mathrm{~mL})$. The organic phase was dried on sodium sulfate, filtered and concentrated under reduced pressure. The compound was obtained as a beige oil (97\% yield) and used without purification. $\mathrm{C}_{16} \mathrm{H}_{17} \mathrm{BrN}_{4} \mathrm{O}_{2} . \mathrm{M}_{\mathrm{W}}: 377.24 \mathrm{~g} / \mathrm{mol} .{ }^{1} \mathrm{H}-\mathrm{NMR} \delta$ (ppm, DMSO-d $\left.d_{6}\right): 1.42(\mathrm{~s}, 9 \mathrm{H}$, $\left.3 \times \mathrm{CH}_{3} \mathrm{OtBu}\right), 4.12\left(\mathrm{~d}, 2 \mathrm{H}, J=8 \mathrm{~Hz}, \mathrm{CH}_{2} \alpha\right), 7.34-7.37(\mathrm{~m}, 1 \mathrm{H}, \mathrm{CH}$ 7), 7.45-7.50 (m, 1H, CH 8), $7.59(\mathrm{dd}$, $1 \mathrm{H}, J=4 \mathrm{~Hz}, J=8 \mathrm{~Hz}, \mathrm{CH}$ 9), $7.76(\mathrm{~s}, 1 \mathrm{H}, \mathrm{CH} 2), 8.02(\mathrm{t}, 1 \mathrm{H}, J=8 \mathrm{~Hz}, \mathrm{NH}), 8.96(\mathrm{dd}, 1 \mathrm{H}, J=4 \mathrm{~Hz}, J=8 \mathrm{~Hz}$, CH 6). ${ }^{13} \mathrm{C}-\mathrm{NMR} \delta$ (ppm, DMSO- $\left.d_{6}\right): 28.22\left(\mathrm{CH}_{3} \mathrm{tBu}\right), 43.26\left(\mathrm{CH}_{2} \alpha\right), 80.93(\mathrm{Cq} \mathrm{tBu}), 99.61(\mathrm{Cq} 1), 115.12$ (CH 6), 123.08 (CH 7), 126.08 (Cq 5a), 127.35 (CH 9), 127.78 (CH 8), 134.01 (Cq 3a), 134.79 (CH 2), 137.63 (Cq 9a), 147.05 (Cq 4), $169.64(\mathrm{C}=\mathrm{O})$. MS (ESI +, QTof, $m / z): 377.0[\mathrm{M}+\mathrm{H}]^{+}$.

Tert-butyl 2-((1-bromoimidazo[1,2-a]quinoxalin-4-yl)amino)propanoate (7b): A solution of $\mathbf{6 b}$ (0.3 $\mathrm{g}$, $0.96 \mathrm{mmol})$ and $\mathrm{N}$-bromosuccinimide $(0.2 \mathrm{~g}, 1.1 \mathrm{mmol})$ in $\mathrm{CHCl}_{3}(50 \mathrm{~mL})$ was heated under reflux for $2 \mathrm{~h}$. The solvent was removed under reduced pressure. The crude mixture was dissolved in ethyl acetate $(50 \mathrm{~mL})$ and successively washed with saturated aqueous ammonium chloride, saturated aqueous sodium bicarbonate, distilled water and finally brine. The organic phase was dried on sodium sulfate, filtered and concentrated under reduced pressure. The compound was obtained as a beige oil (98\% yield) and used without purification. $\mathrm{C}_{17} \mathrm{H}_{19} \mathrm{BrN}_{4} \mathrm{O}_{2} . \mathrm{M}_{\mathrm{W}}: 391.26 \mathrm{~g} / \mathrm{mol} .{ }^{1} \mathrm{H}-\mathrm{NMR} \delta$ (ppm, DMSO- $\left.d_{6}\right): 1.41\left(\mathrm{~s}, 9 \mathrm{H}, 3 \times \mathrm{CH}_{3} \mathrm{OtBu}\right), 1.51\left(\mathrm{~d}, 3 \mathrm{H}, J=8 \mathrm{~Hz}, \mathrm{CH}_{3} \beta\right), 4.55-4.59(\mathrm{~m}, 1 \mathrm{H}, \mathrm{CH} \alpha), 7.34-7.38$ (m, 1H, CH 7), 7.46-7.48 (m, 1H, CH 8), $7.60(\mathrm{dd}, 1 \mathrm{H}, J=4 \mathrm{~Hz}, J=8 \mathrm{~Hz}, \mathrm{CH}$ 9), 7.76 (s, 1H, CH 2), 7.82 $(\mathrm{d}, 1 \mathrm{H}, J=4 \mathrm{~Hz}, \mathrm{NH}), 8.97(\mathrm{dd}, 1 \mathrm{H}, J=4 \mathrm{~Hz}, J=8 \mathrm{~Hz}, \mathrm{CH} 6) .{ }^{13} \mathrm{C}-\mathrm{NMR} \delta\left(\mathrm{ppm}, \mathrm{DMSO}-d_{6}\right): 17.40\left(\mathrm{CH}_{3}\right.$ $\beta), 28.03\left(\mathrm{CH}_{3} \mathrm{tBu}\right), 50.24(\mathrm{CH} \alpha), 80.70(\mathrm{Cq} \mathrm{tBu}), 99.68(\mathrm{CH} 1), 115.06(\mathrm{CH} 6), 123.17(\mathrm{CH} 7), 126.06(\mathrm{Cq}$ 5a), 127.30 (CH 9), 127.45 (CH 8), 133.87 (Cq 3a), 134.73 (CH 2), 137.47 (Cq 9a), 146.58 (Cq 4), 172.66 $(\mathrm{C}=\mathrm{O})$. MS (ESI +, QTof, $m / z): 391.1[\mathrm{M}+\mathrm{H}]^{+}$. 
Tert-butyl 2-((1-bromoimidazo[1,2-a]quinoxalin-4-yl)amino)-3-methylbutanoate (7c): A solution of $\mathbf{6 c}$ $(0.38 \mathrm{~g}, 1.1 \mathrm{mmol})$ and $\mathrm{N}$-bromosuccinimide $(0.24 \mathrm{~g}, 1.4 \mathrm{mmol})$ in $\mathrm{CHCl}_{3}(50 \mathrm{~mL})$ was heated under reflux for $2 \mathrm{~h}$. The solvent was removed under reduced pressure. The crude mixture was dissolved in ethyl acetate $(50 \mathrm{~mL})$ and successively washed with saturated aqueous ammonium chloride, saturated aqueous sodium bicarbonate, distilled water and finally brine. The organic phase was dried on sodium sulfate, filtered and concentrated under reduced pressure. The compound was obtained as a beige oil $\left(96 \%\right.$ yield) and used without purification. $\mathrm{C}_{19} \mathrm{H}_{23} \mathrm{BrN}_{4} \mathrm{O}_{2} . \mathrm{M}_{\mathrm{W}}: 419.31 \mathrm{~g} / \mathrm{mol} .{ }^{1} \mathrm{H}-\mathrm{NMR} \delta$ (ppm, DMSO- $\left.d_{6}\right): 1.02\left(\mathrm{~d}, 3 \mathrm{H}, J=8 \mathrm{~Hz}, \mathrm{CH}_{3} \gamma\right), 1.05\left(\mathrm{~d}, 3 \mathrm{H}, J=8 \mathrm{~Hz}, \mathrm{CH}_{3} \gamma^{\prime}\right), 1.43\left(\mathrm{~s}, 9 \mathrm{H}, 3 \times \mathrm{CH}_{3} \mathrm{OtBu}\right)$, 2.31-2.36 (m, 1H, CH $\beta), 4.49(\mathrm{t}, 1 \mathrm{H}, J=16 \mathrm{~Hz}, \mathrm{CH} \alpha), 7.20(\mathrm{~d}, 1 \mathrm{H}, J=8 \mathrm{~Hz}, \mathrm{NH}), 7.35-7.40(\mathrm{~m}, 1 \mathrm{H}, \mathrm{CH}$ 7), 7.47-7.49 (m, 1H, CH 8), $7.62(\mathrm{dd}, 1 \mathrm{H}, J=4 \mathrm{~Hz}, J=8 \mathrm{~Hz}, \mathrm{CH} 9), 7.76(\mathrm{~s}, 1 \mathrm{H}, \mathrm{CH} 2), 8.97(\mathrm{dd}, 1 \mathrm{H}, J=4$ $\mathrm{Hz}, J=8 \mathrm{~Hz}, \mathrm{CH} 6) .{ }^{13} \mathrm{C}-\mathrm{NMR} \delta\left(\mathrm{ppm}, \mathrm{DMSO}-d_{6}\right): 19.42\left(\mathrm{CH}_{3} \gamma, \mathrm{CH}_{3} \gamma^{\prime}\right), 28.17\left(\mathrm{CH}_{3} \mathrm{tBu}\right), 30.40(\mathrm{CH}$ ß), $59.76(\mathrm{CH} \alpha), 81.25(\mathrm{Cq} \mathrm{tBu}), 99.86$ (Cq 1), 115.08 (CH 6), 123.38 (CH 7), 126.16 (Cq 5a), $127.44(\mathrm{CH}$ 9), 127.47 (CH 8), 133.81 (Cq 3a), 134.74 (CH 2), 137.40 (Cq 9a), 146.84 (Cq 4), 171.44 (C=O). MS (ESI +, QTof, $m / z): 419.1[\mathrm{M}+\mathrm{H}]^{+}$.

Tert-butyl 2-((1-bromoimidazo[1,2-a]quinoxalin-4-yl)amino)-4-methylpentanoate (7d): A solution of $\mathbf{6 d}$ $(0.35 \mathrm{~g}, 1.0 \mathrm{mmol})$ and $\mathrm{N}$-bromosuccinimide $(0.2 \mathrm{~g}, 1.2 \mathrm{mmol})$ in $\mathrm{CHCl}_{3}(50 \mathrm{~mL})$ was heated under reflux for $2 \mathrm{~h}$. The solvent was removed under reduced pressure. The crude mixture was dissolved in ethyl acetate $(50 \mathrm{~mL})$ and successively washed with saturated aqueous ammonium chloride, saturated aqueous sodium bicarbonate, distilled water and finally brine. The organic phase was dried on sodium sulfate, filtered and concentrated under reduced pressure. The compound was obtained as a beige oil ( $95 \%$ yield) and used without purification. $\mathrm{C}_{20} \mathrm{H}_{25} \mathrm{BrN}_{4} \mathrm{O}_{2} . \mathrm{M}_{\mathrm{W}}: 433.34 \mathrm{~g} / \mathrm{mol} .{ }^{1} \mathrm{H}-\mathrm{NMR} \delta$ (ppm, DMSO- $\left.d_{6}\right): 0.91\left(\mathrm{~d}, 3 \mathrm{H}, J=8 \mathrm{~Hz}, \mathrm{CH}_{3} \delta\right), 0.95\left(\mathrm{~d}, 3 \mathrm{H}, J=8 \mathrm{~Hz}, \mathrm{CH}_{3} \delta^{\prime}\right), 1.40\left(\mathrm{~s}, 9 \mathrm{H}, 3 \times \mathrm{CH}_{3} \mathrm{OtBu}\right)$, 1.60-1.62 (m, 1H, $\left.\mathrm{CH}_{2} \beta\right), 1.77-1.79(\mathrm{~m}, 1 \mathrm{H}, \mathrm{CH} \gamma), 1.94-1.96\left(\mathrm{~m}, 1 \mathrm{H}, \mathrm{CH}_{2} \beta\right), 4.60(\mathrm{t}, 1 \mathrm{H}, J=4 \mathrm{~Hz}, \mathrm{CH}$ $\alpha), 7.35-7.38(\mathrm{~m}, 1 \mathrm{H}, \mathrm{CH} 7), 7.46-7.50(\mathrm{~m}, 1 \mathrm{H}, \mathrm{CH} 8), 7.60(\mathrm{dd}, 1 \mathrm{H}, J=4 \mathrm{~Hz}, J=8 \mathrm{~Hz}, \mathrm{CH} 9), 7.71(\mathrm{~d}, 1 \mathrm{H}$, $J=4 \mathrm{~Hz}, \mathrm{NH}), 7.76(\mathrm{~s}, 1 \mathrm{H}, \mathrm{CH} 2), 8.97(\mathrm{dd}, 1 \mathrm{H}, J=4 \mathrm{~Hz}, J=8 \mathrm{~Hz}, \mathrm{CH} 6) .{ }^{13} \mathrm{C}-\mathrm{NMR} \delta\left(\mathrm{ppm}, \mathrm{DMSO}-d_{6}\right)$ : $22.03\left(\mathrm{CH}_{3} \delta\right), 23.31\left(\mathrm{CH}_{3} \delta^{\prime}\right), 25.12(\mathrm{CH} \gamma), 28.14\left(\mathrm{CH}_{3} \mathrm{tBu}\right), 40.10\left(\mathrm{CH}_{2} \beta\right), 52.95(\mathrm{CH} \alpha), 80.76(\mathrm{Cq}$ tBu), 99.21 (Cq 1), 115.07 (CH 6), 123.17 (CH 7), 126.08 (Cq 5a), $127.37(\mathrm{CH} 9), 127.46(\mathrm{CH} \mathrm{8),} 133.88(\mathrm{Cq}$ 3a), 134.71 (CH 2), 137.55 (Cq 9a), 146.97 (Cq 4), 172.59 (C=O). MS (ESI +, QTof, $m / z): 433.1[\mathrm{M}+\mathrm{H}]^{+}$.

Tert-butyl 2-((1-bromoimidazo[1,2-a]quinoxalin-4-yl)amino)-6-((Tert-butoxycarbonyl)amino)-hexanoate (7e): A solution of $6 \mathrm{e}(1.2 \mathrm{~g}, 2.5 \mathrm{mmol})$ and $\mathrm{N}$-bromosuccinimide $(0.54 \mathrm{~g}, 3.1 \mathrm{mmol})$ in $\mathrm{CHCl}_{3}(50 \mathrm{~mL})$ was heated under reflux for $2 \mathrm{~h}$. The solvent was removed under reduced pressure. The crude mixture was dissolved in ethyl acetate $(50 \mathrm{~mL})$ and successively washed with saturated aqueous ammonium chloride, saturated aqueous sodium bicarbonate, distilled water and finally brine. The organic phase was dried on sodium sulfate, filtered and concentrated under reduced pressure. The compound was obtained as a beige oil (78\% yield) and used without purification. $\mathrm{C}_{25} \mathrm{H}_{34} \mathrm{BrN}_{5} \mathrm{O}_{4} \cdot \mathrm{M}_{\mathrm{W}}: 548.47 \mathrm{~g} / \mathrm{mol}$. ${ }^{1} \mathrm{H}-\mathrm{NMR} \delta$ (ppm, DMSO- $\left.d_{6}\right): 1.22-1.24\left(\mathrm{~m}, 2 \mathrm{H}, \mathrm{CH}_{2} \gamma\right), 1.34\left(\mathrm{~s}, 9 \mathrm{H}, 3 \times \mathrm{CH}_{3} \mathrm{OtBu}\right), 1.40\left(\mathrm{~s}, 9 \mathrm{H}, 3 \times \mathrm{CH}_{3}\right.$ $\mathrm{OtBu}), 1.54-1.56\left(\mathrm{~m}, 2 \mathrm{H}, \mathrm{CH}_{2} \beta\right), 1.89-1.91\left(\mathrm{~m}, 2 \mathrm{H}, \mathrm{CH}_{2} \delta\right), 2.83-2.89\left(\mathrm{~m}, 2 \mathrm{H}, \mathrm{CH}_{2} \varepsilon\right), 4.13-4.19(\mathrm{~m}, 1 \mathrm{H}$, $\mathrm{CH} \alpha), 6.78\left(\mathrm{t}, 1 \mathrm{H}, J=4 \mathrm{~Hz}, \mathrm{NH}-\mathrm{CH}_{2} \varepsilon\right), 7.34-7.39(\mathrm{~m}, 1 \mathrm{H}, \mathrm{CH}$ 7), 7.46-7.48 (m, 1H, CH 8), $7.65(\mathrm{dd}, 1 \mathrm{H}$, $J=4 \mathrm{~Hz}, J=8 \mathrm{~Hz}, \mathrm{CH} 9), 8.03(\mathrm{~s}, 1 \mathrm{H}, \mathrm{CH} 2), 8.34(\mathrm{~d}, 1 \mathrm{H}, J=4 \mathrm{~Hz}, \mathrm{NH}-\mathrm{CH} \alpha), 8.97(\mathrm{dd}, 1 \mathrm{H}, J=4 \mathrm{~Hz}$, $J=8 \mathrm{~Hz}, \mathrm{CH} 6) .{ }^{13} \mathrm{C}-\mathrm{NMR} \delta\left(\mathrm{ppm}, \mathrm{DMSO}-d_{6}\right): 22.90\left(\mathrm{CH}_{2} \gamma\right), 28.15\left(\mathrm{CH}_{3} \mathrm{tBu}\right), 28.73\left(\mathrm{CH}_{3} \mathrm{tBu}\right), 29.46$ $\left(\mathrm{CH}_{2} \delta\right), 31.34\left(\mathrm{CH}_{2} \beta\right), 39.36\left(\mathrm{CH}_{2} \varepsilon\right), 54.61(\mathrm{CH} \alpha), 80.85(\mathrm{Cq} \mathrm{tBu}), 81.14(\mathrm{Cq} \mathrm{tBu}), 99.70(\mathrm{Cq} 1), 115.06$ (CH 6), 123.19 (CH 7), 126.09 (Cq 5a), 127.39 (CH 9), 127.46 (CH 8), 133.87 (CH 2), 134.70 (Cq 3a), 137.54 (Cq 9a), 146.88 (Cq 4), $171.39(\mathrm{C}=\mathrm{O}), 172.21(\mathrm{C}=\mathrm{O})$. MS (ESI +, QTof, $m / z): 548.1[\mathrm{M}+\mathrm{H}]^{+}$.

Tert-butyl 5-(((benzyloxy)carbonyl)amino)-2-((1-bromoimidazo[1,2-a]quinoxalin-4-yl)-amino)pentanoate (7f): A solution of $6 \mathbf{f}(0.45 \mathrm{~g}, 0.9 \mathrm{mmol})$ and $\mathrm{N}$-bromosuccinimide $(0.20 \mathrm{~g}, 1.1 \mathrm{mmol})$ in $\mathrm{CHCl}_{3}(50 \mathrm{~mL})$ was heated under reflux for $2 \mathrm{~h}$. The solvent was removed under reduced pressure. The crude mixture was dissolved in ethyl acetate $(50 \mathrm{~mL}$ ) and successively washed with saturated aqueous ammonium chloride, saturated aqueous sodium bicarbonate, distilled water and finally brine. The organic phase was dried on sodium sulfate, filtered and concentrated under reduced pressure. The compound was 
obtained as a beige oil ( $94 \%$ yield) and used without purification. $\mathrm{C}_{27} \mathrm{H}_{30} \mathrm{BrN}_{5} \mathrm{O}_{4} \cdot \mathrm{M}_{\mathrm{W}}: 568.46 \mathrm{~g} / \mathrm{mol}$. ${ }^{1} \mathrm{H}-\mathrm{NMR} \delta\left(\mathrm{ppm}, \mathrm{DMSO}-d_{6}\right): 1.40\left(\mathrm{~s}, 9 \mathrm{H}, 3 \times \mathrm{CH}_{3} \mathrm{OtBu}\right), 1.51-1.59\left(\mathrm{~m}, 2 \mathrm{H}, \mathrm{CH}_{2} \gamma\right), 1.89-1.92(\mathrm{~m}$, $\left.2 \mathrm{H}, \mathrm{CH}_{2} \beta\right), 3.03-3.08\left(\mathrm{~m}, 2 \mathrm{H}, \mathrm{CH}_{2} \delta\right), 4.52-4.54(\mathrm{~m}, 1 \mathrm{H}, \mathrm{CH} \alpha), 5.00\left(\mathrm{~s}, 2 \mathrm{H}, \mathrm{CH}_{2}-\mathrm{Phenyl}, 7.26(\mathrm{~s}, 1 \mathrm{H}\right.$, NH-CH $\left.{ }_{2} \delta\right), 7.31-7.35(\mathrm{~m}, 5 \mathrm{H}, \mathrm{CH}$ Phenyl), 7.36-7.39 (m, 1H, CH 7), 7.46-7.50 (m, 1H, CH 8), 7.59 (dd, $1 \mathrm{H}, J=4 \mathrm{~Hz}, J=8 \mathrm{~Hz}, \mathrm{CH}$ 9), $7.67(\mathrm{~d}, 1 \mathrm{H}, J=4 \mathrm{~Hz}, \mathrm{NH}-\mathrm{CH} \alpha), 7.76(\mathrm{~s}, 1 \mathrm{H}, \mathrm{CH} 2), 8.99(\mathrm{dd}, 1 \mathrm{H}, J=4 \mathrm{~Hz}$, $J=8 \mathrm{~Hz}, \mathrm{CH}$ 6). ${ }^{13} \mathrm{C}-\mathrm{NMR} \delta\left(\mathrm{ppm}, \mathrm{DMSO}-d_{6}\right): 26.53\left(\mathrm{CH}_{2} \gamma\right), 28.13\left(\mathrm{CH}_{3} \mathrm{tBu}\right), 28.50\left(\mathrm{CH}_{2} \beta\right), 40.42$ $\left(\mathrm{CH}_{2} \delta\right), 54.43(\mathrm{CH} \alpha), 65.59$ ( $\mathrm{CH}_{2}$-Phenyl), $80.92(\mathrm{Cq} \mathrm{tBu}), 99.69$ (Cq 1), 115.07 (CH 6), 123.20 (CH 7), 126.10 (Cq 5a), 127.39 (CH 9), 127.45 (CH 8), 128.18 (CH Phenyl), 128.78 (CH Phenyl), 133.87 (Cq 3a), 134.70 (CH 2), 137.72 (Cq 9a), 146.85 (Cq 4), 156.56 (Cq Phenyl), 172.05 (C=O). MS (ESI +, QTof, m/z): $568.3[\mathrm{M}+\mathrm{H}]^{+}$.

Tert-butyl 2-((1-bromoimidazo[1,2-a]quinoxalin-4-yl)amino)-3-phenylpropanoate (7g): A solution of $6 \mathbf{g}(0.34 \mathrm{~g}$, $0.9 \mathrm{mmol})$ and $\mathrm{N}$-bromosuccinimide $(0.18 \mathrm{~g}, 1.1 \mathrm{mmol})$ in $\mathrm{CHCl}_{3}(50 \mathrm{~mL})$ was heated under reflux for 2 $\mathrm{h}$. The solvent was removed under reduced pressure. The crude mixture was dissolved in ethyl acetate $(50 \mathrm{~mL})$ and successively washed with saturated aqueous ammonium chloride, saturated aqueous sodium bicarbonate, distilled water and finally brine. The organic phase was dried on sodium sulfate, filtered and concentrated under reduced pressure. The compound was obtained as a beige oil ( $83 \%$ yield) and used without purification. $\mathrm{C}_{23} \mathrm{H}_{23} \mathrm{BrN}_{4} \mathrm{O}_{2} . \mathrm{M}_{\mathrm{W}}: 467.36 \mathrm{~g} / \mathrm{mol} .{ }^{1} \mathrm{H}-\mathrm{NMR} \delta$ (ppm, DMSO- $d_{6}$ ): $1.33\left(\mathrm{~s}, 9 \mathrm{H}, 3 \times \mathrm{CH}_{3} \mathrm{OtBu}\right), 3.18-3.22\left(\mathrm{~m}, 1 \mathrm{H}, \mathrm{CH}_{2} \beta\right), 3.31-3.34\left(\mathrm{~m}, 1 \mathrm{H}, \mathrm{CH}_{2} \beta\right), 4.79-4.81(\mathrm{~m}, 1 \mathrm{H}$, $\mathrm{CH} \alpha), 7.21(\mathrm{t}, 1 \mathrm{H}, J=8 \mathrm{~Hz}, \mathrm{CH}$ Phenyl), 7.27-7.30 (m, 2H, $2 \times \mathrm{CH}$ Phenyl), 7.32-7.34 (m, 2H, 2xCH Phenyl), 7.35-7.38 (m, 1H, CH 7), 7.46-7.49 (m, 1H, CH 8), 7.60 (dd, 1H, J = 4 Hz, J = 8 Hz, CH 9), 7.65 $(\mathrm{d}, 1 \mathrm{H}, J=8 \mathrm{~Hz}, \mathrm{NH}), 7.75(\mathrm{~s}, 1 \mathrm{H}, \mathrm{CH} 2), 8.97(\mathrm{dd}, 1 \mathrm{H}, J=2 \mathrm{~Hz}, J=8 \mathrm{~Hz}, \mathrm{CH} 6) .{ }^{13} \mathrm{C}-\mathrm{NMR} \delta(\mathrm{ppm}$, DMSO- $\left.d_{6}\right): 28.04\left(\mathrm{CH}_{3} \mathrm{tBu}\right), 36.91\left(\mathrm{CH}_{2} \beta\right), 56.01(\mathrm{CH} \alpha), 81.06(\mathrm{Cq} \mathrm{tBu}), 99.76(\mathrm{Cq} 1), 115.07(\mathrm{CH} 6)$, 123.33 (CH 7), 126.13 (Cq 5a), 126.96 (CH Phenyl), 127.45 (CH 9), 127.47 (CH 8), 128.68 (CH Phenyl), 129.77 (CH Phenyl), 133.80 (Cq Phenyl), 134.76 (CH 2), 137.45 (Cq 3a), 138.05 (Cq 9a), 146.66 (Cq 4), $171.45(\mathrm{C}=\mathrm{O})$. MS (ESI +, QTof, $\mathrm{m} / \mathrm{z}): 467.1[\mathrm{M}+\mathrm{H}]^{+}$.

Tert-butyl 2-((1-bromoimidazo[1,2-a]quinoxalin-4-yl)amino)-3-(4-(Tert-butoxy)phenyl)-propanoate (7h): A solution of $6 \mathbf{h}(0.34 \mathrm{~g}, 0.9 \mathrm{mmol})$ and $\mathrm{N}$-bromosuccinimide $(0.18 \mathrm{~g}, 1.1 \mathrm{mmol})$ in $\mathrm{CHCl}_{3}(50 \mathrm{~mL})$ was heated under reflux for $2 \mathrm{~h}$. The solvent was removed under reduced pressure. The crude mixture was dissolved in ethyl acetate $(50 \mathrm{~mL})$ and successively washed with saturated aqueous ammonium chloride, saturated aqueous sodium bicarbonate, distilled water and finally brine. The organic phase was dried on sodium sulfate, filtered and concentrated under reduced pressure. The compound was obtained as a beige oil (91\% yield) and used without purification. $\mathrm{C}_{23} \mathrm{H}_{23} \mathrm{BrN}_{4} \mathrm{O}_{2} . \mathrm{M}_{\mathrm{W}}: 467.36 \mathrm{~g} / \mathrm{mol}$. ${ }^{1} \mathrm{H}-\mathrm{NMR} \delta\left(\mathrm{ppm}\right.$, DMSO- $d_{6}$ ): $1.22\left(\mathrm{~s}, 9 \mathrm{H}, 3 \times \mathrm{CH}_{3} \mathrm{OtBu}\right), 1.31\left(\mathrm{~s}, 9 \mathrm{H}, 3 \times \mathrm{CH}_{3} \mathrm{OtBu}\right), 3.11-3.16(\mathrm{~m}$, $\left.1 \mathrm{H}, \mathrm{CH}_{2} \beta\right), 3.24-3.26\left(\mathrm{~m}, 1 \mathrm{H}, \mathrm{CH}_{2} \beta\right), 4.76-4.81(\mathrm{~m}, 1 \mathrm{H}, \mathrm{CH} \alpha), 6.85(\mathrm{dd}, 2 \mathrm{H}, J=4 \mathrm{~Hz}, J=8 \mathrm{~Hz}, \mathrm{CH}$ Phenyl), 7.20 (dd, 2H, J = 4 Hz, J = 8 Hz, CH Phenyl), 7.34-7.38 (m, 1H, CH 7), 7.46-7.50 (m, 1H, CH 8), $7.59(\mathrm{dd}, 1 \mathrm{H}, J=4 \mathrm{~Hz}, J=8 \mathrm{~Hz}, \mathrm{CH}$ 9), $7.65(\mathrm{~d}, 1 \mathrm{H}, J=8 \mathrm{~Hz}, \mathrm{NH}), 7.75(\mathrm{~d}, 1 \mathrm{H}, J=4 \mathrm{~Hz}, \mathrm{CH} 2), 8.98(\mathrm{dd}$, $1 \mathrm{H}, J=4 \mathrm{~Hz}, J=8 \mathrm{~Hz}, \mathrm{CH} 6) .{ }^{13} \mathrm{C}-\mathrm{NMR} \delta\left(\mathrm{ppm}, \mathrm{DMSO}-d_{6}\right): 28.04\left(\mathrm{CH}_{3} \mathrm{tBu}\right), 28.93\left(\mathrm{CH}_{3} \mathrm{tBu}\right), 36.61$ $\left(\mathrm{CH}_{2} \beta\right), 56.03(\mathrm{CH} \alpha), 78.10(\mathrm{Cq} \mathrm{tBu}), 81.01(\mathrm{Cq} \mathrm{tBu}), 99.71(\mathrm{Cq} 1), 115.08(\mathrm{CH} 6), 123.32(\mathrm{CH} 7), 123.96$ ( $2 \times \mathrm{CH}$ Phenyl), 126.13 (Cq 5a), 127.42 (CH 9), 127.47 (CH 8), 130.33 (2 $\times$ CH Phenyl), 132.54 (Cq 3a), 134.78 (CH 2), 137.43 (Cq 9a), 146.61 (Cq 4), 154.06 ( $2 \times$ Cq Phenyl), 171.53 (C=O). MS (ESI +, QTof, $\mathrm{m} / \mathrm{z}): 467.1[\mathrm{M}+\mathrm{H}]^{+}$.

Tert-butyl 5-((1-bromoimidazo[1,2-a]quinoxalin-4-yl)amino)-2-((Tert-butoxycarbonyl)amino)-pentanoate (7i): A solution of $\mathbf{6 i}(0.49 \mathrm{~g}, 1.1 \mathrm{mmol})$ and $\mathrm{N}$-bromosuccinimide $(0.23 \mathrm{~g}, 1.3 \mathrm{mmol})$ in $\mathrm{CHCl}_{3}(50 \mathrm{~mL})$ was heated under reflux for $2 \mathrm{~h}$. The solvent was removed under reduced pressure. The crude mixture was dissolved in ethyl acetate $(50 \mathrm{~mL})$ and successively washed with saturated aqueous ammonium chloride, saturated aqueous sodium bicarbonate, distilled water and finally brine. The organic phase was dried on sodium sulfate, filtered and concentrated under reduced pressure. The compound was obtained as a beige oil (92\% yield) and used without purification. $\mathrm{C}_{24} \mathrm{H}_{32} \mathrm{BrN}_{5} \mathrm{O}_{4} \cdot \mathrm{M}_{\mathrm{W}}: 534.45 \mathrm{~g} / \mathrm{mol}$. ${ }^{1} \mathrm{H}-\mathrm{NMR} \delta$ (ppm, DMSO- $\left.d_{6}\right): 1.44$ (s, 9H, CH-COOtBu), 1.46 (s, 9H, NH-COOtBu), $1.79-1.83$ (m, 2H, 
$\left.\mathrm{CH}_{2} \beta\right), 1.91-1.94\left(\mathrm{~m}, 2 \mathrm{H}, \mathrm{CH}_{2} \gamma\right), 3.73-3.78\left(\mathrm{~m}, 2 \mathrm{H}, \mathrm{CH}_{2} \delta\right), 4.25-4.29(\mathrm{~m}, 1 \mathrm{H}, \mathrm{CH} \alpha), 5.39(\mathrm{~d}, 1 \mathrm{H}, J=4$ $\mathrm{Hz}, \mathrm{NH}-\mathrm{CH} \alpha), 6.33\left(\mathrm{~d}, 1 \mathrm{H}, J=4 \mathrm{~Hz}, \mathrm{NH}-\mathrm{CH}_{2} \delta\right), 7.27-7.31(\mathrm{~m}, 1 \mathrm{H}, \mathrm{CH}$ 7), 7.41-7.45 (m, 1H, CH 8), $7.48(\mathrm{~s}, 1 \mathrm{H}, \mathrm{CH}$ 2), $7.81(\mathrm{dd}, 1 \mathrm{H}, J=4 \mathrm{~Hz}, J=8 \mathrm{~Hz}, \mathrm{CH}$ 9), $8.98(\mathrm{dd}, 1 \mathrm{H}, J=4 \mathrm{~Hz}, J=8 \mathrm{~Hz}, \mathrm{CH} 6)$. ${ }^{13} \mathrm{C}-\mathrm{NMR} \delta$ (ppm, DMSO- $\left.d_{6}\right): 25.74\left(\mathrm{CH}_{2} \beta\right), 28.07\left(\mathrm{CH}_{3} \mathrm{tBu}\right), 28.45\left(\mathrm{CH}_{3} \mathrm{tBu}\right), 30.17\left(\mathrm{CH}_{2} \gamma\right), 40.42$ $\left(\mathrm{CH}_{2} \delta\right), 54.00(\mathrm{CH} \alpha), 79.75(\mathrm{Cq} \mathrm{tBu}), 81.98(\mathrm{Cq} \mathrm{tBu}), 99.05$ (Cq 1), $115.02(\mathrm{CH} 6), 122.77(\mathrm{CH} \mathrm{7}), 124.54$ (Cq 5a), 127.12 (CH 8), 127.98 (CH 9), 134.22 (CH 2), 135.98 (Cq 3a), 146.86 (Cq 4), 155.59 (Cq 9a), 171.92 $(\mathrm{C}=\mathrm{O})$. MS (ESI +, QTof, $m / z): 534.1[\mathrm{M}+\mathrm{H}]^{+}$.

\subsubsection{Suzuki-Miyaura Cross-Coupling Reactions}

Tert-butyl 2-((1-(3,4-dimethoxyphenyl)imidazo[1,2-a]quinoxalin-4-yl)amino)acetate (8a): To a mixture of $7 \mathrm{a}(0.270 \mathrm{~g}, 0.71 \mathrm{mmol})$ in $\mathrm{DME} / \mathrm{H}_{2} \mathrm{O}(2 / 1,15 \mathrm{~mL})$ were added 3,4-dimethoxyphenylboronic acid $(0.143 \mathrm{~g}, 0.79 \mathrm{mmol})$, tetrakis(triphenylphosphine) palladium $(0.042 \mathrm{~g}, 0.036 \mathrm{mmol})$ and sodium carbonate $(0.152 \mathrm{~g}, 1.43 \mathrm{mmol})$ in a microwave-adapted vial. The reaction was submitted to microwave irradiations during $20 \mathrm{~min}$ at $150^{\circ} \mathrm{C}$ and then filtered on a Celite pad. The filtrate was concentrated under reduced pressure and successively washed with saturated aqueous ammonium chloride, saturated aqueous sodium bicarbonate, distilled water and finally brine. The organic phase was dried on sodium sulfate, filtered and concentrated under reduced pressure. The product was purified by flash chromatography eluted with cyclohexane/ethyl acetate $80 / 20$ to $50 / 50$. The compound is obtained as a white solid (19\% yield). $\mathrm{C}_{24} \mathrm{H}_{26} \mathrm{~N}_{4} \mathrm{O}_{4}$. MW: $434.49 \mathrm{~g} / \mathrm{mol} .{ }^{1} \mathrm{H}-\mathrm{NMR} \delta$ (ppm, DMSO- $d_{6}$ ): $1.44\left(\mathrm{~s}, 9 \mathrm{H}, 3 \times \mathrm{CH}_{3} \mathrm{OtBu}\right), 3.74\left(\mathrm{~s}, 3 \mathrm{H}, \mathrm{OCH}_{3}\right.$ Phenyl), $3.87\left(\mathrm{~s}, 3 \mathrm{H}, \mathrm{OCH}_{3}\right.$ Phenyl), $4.14(\mathrm{~d}, 2 \mathrm{H}, J=8 \mathrm{~Hz}$, $\left.\mathrm{CH}_{2} \alpha\right), 7.01-7.05(\mathrm{~m}, 1 \mathrm{H}, \mathrm{CH}$ 7), $7.11(\mathrm{~d}, 1 \mathrm{H}, J=4 \mathrm{~Hz}, \mathrm{CH}$ Phenyl), $7.14(\mathrm{~s}, 1 \mathrm{H}, J=4 \mathrm{~Hz}, \mathrm{CH}$ Phenyl), $7.18(\mathrm{~d}, 1 \mathrm{H}, J=4 \mathrm{~Hz}, \mathrm{CH}$ Phenyl), $7.31(\mathrm{dd}, 1 \mathrm{H}, J=4 \mathrm{~Hz}, J=8 \mathrm{~Hz}, \mathrm{CH} 6), 7.33-7.35(\mathrm{~m}, 1 \mathrm{H}, \mathrm{CH}$ 8), 7.54 (s, 1H, CH 2), $7.56(\mathrm{dd}, 1 \mathrm{H}, J=4 \mathrm{~Hz}, J=8 \mathrm{~Hz}, \mathrm{CH} 9), 7.96(\mathrm{t}, 1 \mathrm{H}, J=8 \mathrm{~Hz}, \mathrm{NH}) .{ }^{13} \mathrm{C}-\mathrm{NMR} \delta$ (ppm, DMSO- $\left.d_{6}\right): 28.26\left(\mathrm{CH}_{3} \mathrm{tBu}\right), 43.33\left(\mathrm{CH}_{2} \alpha\right), 80.86(\mathrm{Cq} \mathrm{tBu}), 112.31$ (CH Phenyl), 114.25 (CH Phenyl), 115.93 (CH 6), 122.75 (CH 7), 123.30 (CH Phenyl), 126.03 (Cq 5a), 126.58 (CH 8), 127.20 (CH 9), 132.66 (CH 2), 133.141 (Cq 3a), 130.93 (Cq 1), 137.71 (Cq 9a), 149.27 (Cq Phenyl), 150.14 (Cq Phenyl), 147.74 (Cq 4), $169.85(\mathrm{C}=\mathrm{O})$. MS (ESI +, QTof, $m / z): 435.1[\mathrm{M}+\mathrm{H}]^{+}$.

Tert-butyl 2-((1-(3,4-dimethoxyphenyl)imidazo[1,2-a]quinoxalin-4-yl)amino)propanoate (8b): Following the same procedure used for the synthesis of $\mathbf{8 a}$, to a mixture of $7 \mathbf{b}(0.440 \mathrm{~g}, 1.12 \mathrm{mmol})$ in $\mathrm{DME} / \mathrm{H}_{2} \mathrm{O}(2 / 1,15 \mathrm{~mL})$ were added 3,4-dimethoxyphenylboronic acid $(0.225 \mathrm{~g}, 1.24 \mathrm{mmol})$, tetrakis(triphenylphosphine) palladium $(0.065 \mathrm{~g}, 0.056 \mathrm{mmol})$ and sodium carbonate $(0.237 \mathrm{~g}$, $2.24 \mathrm{mmol}$ ) in a microwave-adapted vial. The product was purified by flash chromatography eluted with cyclohexane/ethyl acetate $80 / 20$ to $50 / 50$. The compound is obtained as a white solid (42\% yield). $\mathrm{C}_{25} \mathrm{H}_{28} \mathrm{~N}_{4} \mathrm{O}_{4}$. MW: $448.51 \mathrm{~g} / \mathrm{mol} .{ }^{1} \mathrm{H}-\mathrm{NMR} \delta$ (ppm, DMSO-d $d_{6}$ ): $1.32\left(\mathrm{~s}, 9 \mathrm{H}, 3 \times \mathrm{CH}_{3} \mathrm{OtBu}\right), 1.42(\mathrm{~d}$, $\left.3 \mathrm{H}, J=8 \mathrm{~Hz}, \mathrm{CH}_{3} \beta\right), 3.64\left(\mathrm{~s}, 3 \mathrm{H}, \mathrm{OCH}_{3}\right.$ Phenyl), $3.76\left(\mathrm{~s}, 3 \mathrm{H}, \mathrm{OCH}_{3}\right.$ Phenyl), 4.48-4.52 (m, $\left.1 \mathrm{H}, \mathrm{CH} \alpha\right)$, 6.90-6.95 (m, 1H, CH 7), 7.01 (d, 1H, J = 4 Hz, CH Phenyl), 7.03 (s, 1H, CH Phenyl), 7.08 (d, 1H, J = 4 $\mathrm{Hz}, \mathrm{CH}$ Phenyl), 7.20 (dd, 1H, J = $4 \mathrm{~Hz}, J=8 \mathrm{~Hz}, \mathrm{CH}$ 6), 7.22-7.25 (m, 1H, CH 8), 7.42 (s, 1H, CH 2), $7.46(\mathrm{dd}, 1 \mathrm{H}, J=4 \mathrm{~Hz}, J=8 \mathrm{~Hz}, \mathrm{CH} 9), 7.82(\mathrm{~d}, 1 \mathrm{H}, J=4 \mathrm{~Hz}, \mathrm{NH}) .{ }^{13} \mathrm{C}-\mathrm{NMR} \delta$ (ppm, DMSO- $\left.d_{6}\right): 17.59$ $\left(\mathrm{CH}_{3} \beta\right), 28.25\left(\mathrm{CH}_{3} \mathrm{tBu}\right), 50.30(\mathrm{CH} \alpha), 80.72(\mathrm{Cq} \mathrm{tBu}), 112.40$ (CH Phenyl), 114.33 (CH Phenyl), 116.00 (CH 6), 122.77 (CH 7), 122.90 (Cq 1), 123.37 (CH Phenyl), 126.10 (CH 8), 126.65 (Cq 5a), 127.28 (CH 9), 131.05 (Cq 3a), 132.63 (CH 2), 137.68 (Cq 9a), 147.33 (Cq 4), 149.36 (Cq Phenyl), 150.22 (Cq Phenyl), $172.96(\mathrm{C}=\mathrm{O})$. MS (ESI +, QTof, $\mathrm{m} / \mathrm{z}): 449.2[\mathrm{M}+\mathrm{H}]^{+}$. HRMS calculated for $\mathrm{C}_{25} \mathrm{H}_{29} \mathrm{~N}_{4} \mathrm{O}_{4} 449.2189$, found 449.2186 .

Tert-butyl 2-((1-(3,4-dimethoxyphenyl)imidazo[1,2-a]quinoxalin-4-yl)amino)-3-methylbutanoate (8c): Followigng the same procedure used for the synthesis of $8 \mathbf{a}$, to a mixture of $7 \mathrm{c}(0.370 \mathrm{~g}, 0.88 \mathrm{mmol})$ in $\mathrm{DME} / \mathrm{H}_{2} \mathrm{O}(2 / 1,15 \mathrm{~mL})$ were added 3,4-dimethoxyphenylboronic acid $(0.176 \mathrm{~g}, 0.97 \mathrm{mmol})$, tetrakis(triphenylphosphine) palladium $(0.051 \mathrm{~g}, 0.044 \mathrm{mmol})$ and sodium carbonate $(0.186 \mathrm{~g}$, $1.76 \mathrm{mmol}$ ) in a microwave-adapted vial. The product was purified by flash chromatography eluted with cyclohexane/ethyl acetate $80 / 20$ to $50 / 50$. The compound is obtained as a white solid (69\% 
yield). $\mathrm{C}_{27} \mathrm{H}_{32} \mathrm{~N}_{4} \mathrm{O}_{4}$. MW: $476.57 \mathrm{~g} / \mathrm{mol} .{ }^{1} \mathrm{H}-\mathrm{NMR} \delta$ (ppm, DMSO- $d_{6}$ ): 0.95 (d, $3 \mathrm{H}, J=8 \mathrm{~Hz}, \mathrm{CH}_{3} \gamma$ ), $0.98\left(\mathrm{~d}, 3 \mathrm{H}, J=8 \mathrm{~Hz}, \mathrm{CH}_{3} \gamma^{\prime}\right), 1.34\left(\mathrm{~s}, 9 \mathrm{H}, 3 \times \mathrm{CH}_{3} \mathrm{OtBu}\right), 2.20-2.28(\mathrm{~m}, 1 \mathrm{H}, \mathrm{CH} \beta), 3.63\left(\mathrm{~s}, 3 \mathrm{H}, \mathrm{OCH}_{3}\right.$ Phenyl), $3.77\left(\mathrm{~s}, 3 \mathrm{H}, \mathrm{OCH}_{3}\right.$ Phenyl), $4.44(\mathrm{t}, 1 \mathrm{H}, J=16 \mathrm{~Hz}, \mathrm{CH} \alpha), 6.92-6.97(\mathrm{~m}, 1 \mathrm{H}, \mathrm{CH}$ 7), $7.00(\mathrm{~s}, 1 \mathrm{H}$, CH Phenyl), $7.02(\mathrm{~d}, 1 \mathrm{H}, J=4 \mathrm{~Hz}, \mathrm{NH}), 7.06(\mathrm{~d}, 1 \mathrm{H}, J=4 \mathrm{~Hz}, \mathrm{CH}$ Phenyl), $7.08(\mathrm{~d}, 1 \mathrm{H}, J=4 \mathrm{~Hz}, \mathrm{CH}$ Phenyl), 7.22 (dd, $1 \mathrm{H}, J=4 \mathrm{~Hz}, J=8 \mathrm{~Hz}, \mathrm{CH}$ 6), 7.23-7.26 (m, 1H, CH 8), 7.42 (s, 1H, CH 2), 7.49 (dd, $1 \mathrm{H}, J=4 \mathrm{~Hz}, J=8 \mathrm{~Hz}, \mathrm{CH} 9) .{ }^{13} \mathrm{C}-\mathrm{NMR} \delta\left(\mathrm{ppm}, \mathrm{DMSO}-d_{6}\right): 19.43\left(\mathrm{CH}_{3} \gamma^{\prime}\right), 19.56\left(\mathrm{CH}_{3} \gamma\right), 28.28\left(\mathrm{CH}_{3}\right.$ $\mathrm{tBu}), 30.57(\mathrm{CH} \beta), 56.15\left(\mathrm{OCH}_{3}\right.$ Phenyl), $56.24\left(\mathrm{OCH}_{3}\right.$ Phenyl), $59.73(\mathrm{CH} \alpha), 81.31(\mathrm{Cq} \mathrm{tBu}), 112.41$ (CH Phenyl), 114.35 (CH Phenyl), 116.02 (CH 6), 122.67 (Cq 1), 123.14 (CH 7), 123.39 (CH Phenyl), 126.19 (Cq 5a), 126.71 (CH 8), 127.39 (CH 9), 131.25 (Cq Phenyl), 132.68 (CH 2), 133.00 (Cq 3a), 137.55 (Cq 9a), 147.56 (Cq 4), 149.36 (Cq Phenyl), 150.26 (Cq Phenyl), 171.70 (C=O). MS (ESI +, QTof, m/z): $477.1[\mathrm{M}+\mathrm{H}]^{+}$. HRMS calculated for $\mathrm{C}_{27} \mathrm{H}_{33} \mathrm{~N}_{4} \mathrm{O}_{4}$ 477.2502, found 477.2505.

Tert-butyl 2-((1-(3,4-dimethoxyphenyl)imidazo[1,2-a]quinoxalin-4-yl)amino)-4-methyl-pentanoate: $\quad$ (8d): Following the same procedure used for the synthesis of $\mathbf{8 a}$, to a mixture of $\mathbf{7 d}(0.360 \mathrm{~g}, 0.83 \mathrm{mmol})$ in $\mathrm{DME} / \mathrm{H}_{2} \mathrm{O}(2 / 1,15 \mathrm{~mL})$ were added 3,4-dimethoxyphenylboronic acid $(0.166 \mathrm{~g}, 0.91 \mathrm{mmol})$, tetrakis(triphenylphosphine) palladium $(0.048 \mathrm{~g}, 0.041 \mathrm{mmol})$ and sodium carbonate $(0.176 \mathrm{~g}, 1.66 \mathrm{mmol})$ in a microwave-adapted vial. The product was purified by flash chromatography eluted with cyclohexane/ethyl acetate $80 / 20$ to $50 / 50$. The compound is obtained as a white solid (61\% yield). $\mathrm{C}_{28} \mathrm{H}_{34} \mathrm{~N}_{4} \mathrm{O}_{4}$. MW: $490.59 \mathrm{~g} / \mathrm{mol} .{ }^{1} \mathrm{H}-\mathrm{NMR} \delta$ (ppm, DMSO- $\left.d_{6}\right): 0.83\left(\mathrm{~d}, 3 \mathrm{H}, J=8 \mathrm{~Hz}, \mathrm{CH}_{3} \delta\right), 0.86(\mathrm{~d}$, $\left.3 \mathrm{H}, J=8 \mathrm{~Hz}, \mathrm{CH}_{3} \delta^{\prime}\right), 1.31\left(\mathrm{~s}, 9 \mathrm{H}, 3 \times \mathrm{CH}_{3} \mathrm{OtBu}\right), 1.55-1.58\left(\mathrm{~m}, 1 \mathrm{H}, \mathrm{CH}_{2} \beta\right), 1.67-1.70(\mathrm{~m}, 1 \mathrm{H}, \mathrm{CH} \gamma)$, 1.84-1.88 (m, $\left.1 \mathrm{H}, \mathrm{CH}_{2} \beta\right), 3.63\left(\mathrm{~s}, 3 \mathrm{H}, \mathrm{OCH}_{3}\right.$ Phenyl), 3.76 (s, 3H, OCH $\mathrm{OCh}_{3}$ Phenyl), $4.55(\mathrm{t}, 1 \mathrm{H}, J=4 \mathrm{~Hz}, \mathrm{CH}$

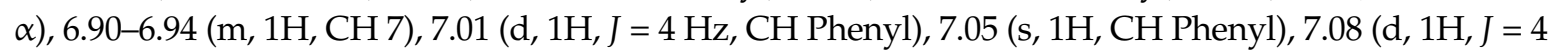
$\mathrm{Hz}, \mathrm{CH}$ Phenyl), 7.20 (dd, $1 \mathrm{H}, J=4 \mathrm{~Hz}, J=8 \mathrm{~Hz}, \mathrm{CH}$ ) $), 7.22-7.24$ (m, 1H, CH 8), 7.41 (s, 1H, CH 2), 7.45 $(\mathrm{dd}, 1 \mathrm{H}, J=4 \mathrm{~Hz}, J=8 \mathrm{~Hz}, \mathrm{CH} 9), 7.50(\mathrm{~d}, 1 \mathrm{H}, J=4 \mathrm{~Hz}, \mathrm{NH}) .{ }^{13} \mathrm{C}-\mathrm{NMR} \delta\left(\mathrm{ppm}, \mathrm{DMSO}-d_{6}\right): 22.14\left(\mathrm{CH}_{3} \delta\right)$, $23.41\left(\mathrm{CH}_{3} \delta^{\prime}\right), 25.25(\mathrm{CH} \gamma), 28.25\left(\mathrm{CH}_{3} \mathrm{tBu}\right), 40.29\left(\mathrm{CH}_{2} \beta\right), 52.96(\mathrm{CH} \alpha), 56.14\left(\mathrm{OCH}_{3}\right.$ Phenyl), 56.23 $\left(\mathrm{OCH}_{3}\right.$ Phenyl), 80.79 (Cq tBu), 112.40 (CH Phenyl), 114.34 (CH Phenyl), 115.99 (CH 6), 122.78 (Cq 1), 122.88 (CH 7), 123.36 (CH Phenyl), 126.11 (Cq 5a), 126.69 (CH 8), 127.29 (CH 9), 131.09 (Cq 3a), 132.61 (CH 2), 137.70 (Cq 9a), 147.66 (Cq 4), 149.36 (Cq Phenyl), 150.23 (Cq Phenyl), 172.86 (C=O). MS (ESI +, QTof, $m / z): 491.0[\mathrm{M}+\mathrm{H}]^{+}$. HRMS calculated for $\mathrm{C}_{28} \mathrm{H}_{35} \mathrm{~N}_{4} \mathrm{O}_{4} 491.2658$, found 491.2654.

Tert-butyl 6-((Tert-butoxycarbonyl)amino)-2-((1-(3,4-dimethoxyphenyl)imidazo[1,2-a]-quinoxalin-4-yl)amino) hexanoate (8e): Following the same procedure used for the synthesis of $\mathbf{8 a}$, to a mixture of $\mathbf{7 e}(1.090 \mathrm{~g}$, $1.99 \mathrm{mmol})$ in DME/ $\mathrm{H}_{2} \mathrm{O}(2 / 1,15 \mathrm{~mL})$ were added 3,4-dimethoxyphenylboronic acid $(0.397 \mathrm{~g}$, $2.18 \mathrm{mmol})$, tetrakis(triphenylphosphine) palladium $(0.114 \mathrm{~g}, 0.099 \mathrm{mmol})$ and sodium carbonate $(0.421 \mathrm{~g}, 3.97 \mathrm{mmol})$ in a microwave-adapted vial. The product was purified by flash chromatography eluted with cyclohexane/ethyl acetate $80 / 20$ to $50 / 50$. The compound is obtained as a white solid (7\% yield). $\mathrm{C}_{33} \mathrm{H}_{43} \mathrm{~N}_{5} \mathrm{O}_{6}$. MW: $605.72 \mathrm{~g} / \mathrm{mol} .{ }^{1} \mathrm{H}-\mathrm{NMR} \delta$ (ppm, DMSO- $d_{6}$ ): 1.36 (s, 9H, $3 \times \mathrm{CH}_{3} \mathrm{OtBu}$ ), 1.41-1.46 (m, 13H, $\left.\mathrm{CH}_{2} \gamma, 3 \times \mathrm{CH}_{3} \mathrm{OtBu}, \mathrm{CH}_{2} \delta\right), 1.88-1.91\left(\mathrm{~m}, 2 \mathrm{H}, \mathrm{CH}_{2} \beta\right), 2.93-2.95\left(\mathrm{~m}, 2 \mathrm{H}, \mathrm{CH}_{2} \varepsilon\right)$, 3.73 (s, 3H, $\mathrm{OCH}_{3}$ Phenyl), 3.87 (s, 3H, OCH 3 Phenyl), 4.54-4.58 (m, $\left.1 \mathrm{H}, \mathrm{CH} \alpha\right), 6.80(\mathrm{t}, 1 \mathrm{H}, J=4 \mathrm{~Hz}$, NH-CH $\left.{ }_{2} \varepsilon\right), 7.01-7.05(\mathrm{~m}, 1 \mathrm{H}, \mathrm{CH} 7), 7.11(\mathrm{~d}, 1 \mathrm{H}, \mathrm{J}=4 \mathrm{~Hz}, \mathrm{CH}$ Phenyl), 7.15 (s, 1H, CH Phenyl), 7.18 $(\mathrm{d}, 1 \mathrm{H}, J=4 \mathrm{~Hz}, \mathrm{CH}$ Phenyl), $7.31(\mathrm{dd}, 1 \mathrm{H}, J=4 \mathrm{~Hz}, J=8 \mathrm{~Hz}, \mathrm{CH}$ 6), 7.31-7.35 (m, 1H, CH 8), $7.52(\mathrm{~s}$, $1 \mathrm{H}, \mathrm{CH}$ 2), $7.55(\mathrm{dd}, 1 \mathrm{H}, J=4 \mathrm{~Hz}, J=8 \mathrm{~Hz}, \mathrm{CH} 9), 7.59(\mathrm{~d}, 1 \mathrm{H}, J=4 \mathrm{~Hz}, \mathrm{NH}-\mathrm{CH} \alpha) .{ }^{13} \mathrm{C}-\mathrm{NMR} \delta(\mathrm{ppm}$, DMSO- $\left.d_{6}\right): 23.49\left(\mathrm{CH}_{2} \gamma\right), 28.18\left(\mathrm{CH}_{3} \mathrm{tBu}\right), 28.72\left(\mathrm{CH}_{3} \mathrm{tBu}\right), 29.64\left(\mathrm{CH}_{2} \delta\right), 30.94\left(\mathrm{CH}_{2} \beta\right), 40.40\left(\mathrm{CH}_{2}\right.$ ع), $54.56(\mathrm{CH} \alpha), 56.05\left(\mathrm{OCH}_{3}\right.$ Phenyl), $56.14\left(\mathrm{OCH}_{3}\right.$ Phenyl), $77.78(\mathrm{Cq} \mathrm{tBu}), 80.80(\mathrm{Cq} \mathrm{tBu}), 112.30(\mathrm{CH}$ Phenyl), 114.22 (CH Phenyl), 115.91 (CH 6), 122.67 (CH 7), 123.28 (CH Phenyl), 126.03 (Cq 5a), 126.56 (CH 8), 127.23 (CH 9), 131.01 (Cq 1), 132.53 (CH 2), 132.98 (Cq 3a), 137.61 (Cq 9a), 147.51 (Cq 4), 149.27 (Cq Phenyl), 150.14 (Cq Phenyl), 156.04 (Cq Phenyl), 172.41 (C=O). MS (ESI +, QTof, $m / z): 606.2$ [M + $\mathrm{H}]^{+}$. HRMS calculated for $\mathrm{C}_{33} \mathrm{H}_{44} \mathrm{~N}_{5} \mathrm{O}_{6}$ 606.3292, found 606.3291.

Tert-butyl 5-(((benzyloxy)carbonyl)amino)-2-((1-(3,4-dimethoxyphenyl)imidazo[1,2-a]-quinoxalin-4-yl)amino) pentanoate (8f): Following the same procedure used for the synthesis of $8 \mathbf{a}$, to a mixture of $\mathbf{7 f}(0.470 \mathrm{~g}$, $0.89 \mathrm{mmol})$ in $\mathrm{DME} / \mathrm{H}_{2} \mathrm{O}(2 / 1,15 \mathrm{~mL})$ were added 3,4-dimethoxyphenylboronic acid $(0.165 \mathrm{~g}$, 
$0.91 \mathrm{mmol})$, tetrakis(triphenylphosphine) palladium $(0.048 \mathrm{~g}, 0.041 \mathrm{mmol})$ and sodium carbonate $(0.175 \mathrm{~g}, 1.65 \mathrm{mmol})$ in a microwave-adapted vial. The product was purified by flash chromatography eluted with cyclohexane/ethyl acetate $80 / 20$ to $50 / 50$. The compound is obtained as a white solid (42\% yield). $\mathrm{C}_{35} \mathrm{H}_{39} \mathrm{~N}_{5} \mathrm{O}_{6}$. MW: $625.71 \mathrm{~g} / \mathrm{mol} .{ }^{1} \mathrm{H}-\mathrm{NMR} \delta$ (ppm, DMSO- $\left.d_{6}\right):{ }^{1} \mathrm{H}-\mathrm{NMR} \delta$ (ppm, 400 MHz, DMSO- $\left.d_{6}\right): 1.52\left(\mathrm{~s}, 9 \mathrm{H}, 3 \times \mathrm{CH}_{3} \mathrm{OtBu}\right), 1.77-1.81\left(\mathrm{~m}, 2 \mathrm{H}, \mathrm{CH}_{2} \gamma\right), 1.99-2.03\left(\mathrm{~m}, 1 \mathrm{H}, \mathrm{CH}_{2} \delta\right)$, 2.10-2.13 (m, 1H, $\left.\mathrm{CH}_{2} \delta\right), 3.34-3.39\left(\mathrm{~m}, 2 \mathrm{H}, \mathrm{CH}_{2} \beta\right), 3.88$ (s, 3H, $\mathrm{OCH}_{3}$ Phenyl), $4.02\left(\mathrm{~s}, 3 \mathrm{H}, \mathrm{OCH}_{3}\right.$ Phenyl), 5.12 (s, 2H, CH 2 -Phenyl), 5.37-5.39 (m, 1H, CH $\alpha$ ), 6.99 (d, 2H, J = 4 Hz, CH Phenyl), 7.03 (s, 1H, CH Phenyl), 7.10-7.12 (m, 1H, CH 7), 7.28 (dd, 1H, J = 4 Hz, J = 8 Hz, CH 6), 7.32-7.35 (m, 7H, CH 8, CH2, CH Phenyl), 7.36 (dd, $1 \mathrm{H}, J=4 \mathrm{~Hz}, J=8 \mathrm{~Hz}, \mathrm{CH}$ 9), 7.47 (d, $1 \mathrm{H}, J=4 \mathrm{~Hz}, \mathrm{NH}-\mathrm{CH}_{2} \delta$ ), 7.76 (dd, $1 \mathrm{H}, J=4 \mathrm{~Hz}, J=8 \mathrm{~Hz}, \mathrm{NH}-\mathrm{CH} \alpha) .{ }^{13} \mathrm{C}-\mathrm{NMR} \delta\left(\mathrm{ppm}, \mathrm{DMSO}-d_{6}\right): 25.62\left(\mathrm{CH}_{2} \gamma\right), 28.09\left(\mathrm{CH}_{3} \mathrm{tBu}\right), 30.04$ $\left(\mathrm{CH}_{2} \beta\right), 40.61\left(\mathrm{CH}_{2} \delta\right), 54.42(\mathrm{CH} \alpha), 66.58\left(\mathrm{CH}_{2}\right.$-Phenyl), 82.42 (Cq tBu), 111.30 (CH Phenyl), 113.24 (CH Phenyl), 114.69 (Cq 1), 115.99 (CH 6), 123.16 (CH 7), 126.40 (Cq 5a), 126.82 (CH 9), 126.87 (CH 8), 128.01 (CH Phenyl), 128.08 (CH Phenyl), 129.49 (CH Phenyl), 131.85 (CH 2), 132.87 (Cq 3a), 136.68 (Cq 9a), 149.16 (Cq 4), 150.12 (Cq Phenyl), 152.37 (Cq Phenyl), 156.51 (Cq Phenyl), 171.59 (C=O). MS (ESI +, QTof, $m / z): 626.0[\mathrm{M}+\mathrm{H}]^{+}$. HRMS calculated for $\mathrm{C}_{35} \mathrm{H}_{40} \mathrm{~N}_{5} \mathrm{O}_{6} 626.2979$, found 626.2982 .

Tert-butyl 2-((Tert-butoxycarbonyl)amino)-5-((1-(3,4-dimethoxyphenyl)imidazo[1,2-a]-quinoxalin-4-yl)amino) pentanoate (8i): Following the same procedure used for the synthesis of $\mathbf{8 a}$, to a mixture of $\mathbf{7 i}(0.515 \mathrm{~g}$, $0.96 \mathrm{mmol})$ in DME $/ \mathrm{H}_{2} \mathrm{O}(2 / 1,15 \mathrm{~mL})$ were added 3,4-dimethoxyphenylboronic acid $(0.193 \mathrm{~g}$, $1.06 \mathrm{mmol})$, tetrakis(triphenylphosphine) palladium $(0.056 \mathrm{~g}, 0.048 \mathrm{mmol})$ and sodium carbonate $(0.204 \mathrm{~g}, 1.93 \mathrm{mmol})$ in a microwave-adapted vial. The product was purified by flash chromatography eluted with cyclohexane/ethyl acetate $80 / 20$ to $50 / 50$. The compound is obtained as a white solid (45\% yield). $\mathrm{C}_{32} \mathrm{H}_{41} \mathrm{~N}_{5} \mathrm{O}_{6}$. MW: $591.70 \mathrm{~g} / \mathrm{mol} .{ }^{1} \mathrm{H}-\mathrm{NMR} \delta$ (ppm, DMSO- $d_{6}$ ): 1.34 (s, 9H, CH-COOtBu), 1.39 (s, 9H, NH-COOtBu), 1.73-1.74 (m, 2H, $\left.\mathrm{CH}_{2} \beta\right), 1.77-1.78\left(\mathrm{~m}, 2 \mathrm{H}, \mathrm{CH}_{2} \gamma\right), 3.56-3.59\left(\mathrm{~m}, 2 \mathrm{H}, \mathrm{CH}_{2}\right.$

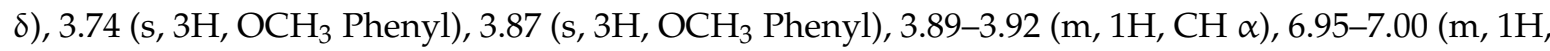
CH 7), 7.09 (dd, 1H, $J=4 \mathrm{~Hz}, J=8 \mathrm{~Hz}, \mathrm{CH}$ Phenyl), 7.14 (s, 1H, CH Phenyl), 7.15 (dd, 1H, $J=4 \mathrm{~Hz}, J=8$ $\mathrm{Hz}, \mathrm{CH}$ Phenyl), $7.19(\mathrm{~d}, 1 \mathrm{H}, J=4 \mathrm{~Hz}, \mathrm{NH}-\mathrm{CH} \alpha), 7.28(\mathrm{dd}, 1 \mathrm{H}, J=4 \mathrm{~Hz}, J=8 \mathrm{~Hz}, \mathrm{CH}$ 6), 7.30-7.32 (m, $1 \mathrm{H}, \mathrm{CH} 8), 7.47\left(\mathrm{~s}, 1 \mathrm{H}, \mathrm{CH}\right.$ 2), $7.58(\mathrm{dd}, 1 \mathrm{H}, J=4 \mathrm{~Hz}, J=8 \mathrm{~Hz} \mathrm{CH} 9), 7.72\left(\mathrm{t}, 1 \mathrm{H}, J=4 \mathrm{~Hz}, \mathrm{NH}_{-} \mathrm{CH}_{2} \delta\right)$. ${ }^{13} \mathrm{C}-\mathrm{NMR} \delta$ (ppm, DMSO- $\left.d_{6}\right): 25.97\left(\mathrm{CH}_{2} \beta\right), 28.06\left(\mathrm{CH}_{3} \mathrm{tBu}\right), 28.25\left(\mathrm{CH}_{3} \mathrm{tBu}\right), 28.67\left(\mathrm{CH}_{2} \gamma\right), 39.79$ $\left(\mathrm{CH}_{2} \delta\right), 54.77(\mathrm{CH} \alpha), 56.05\left(\mathrm{OCH}_{3}\right.$ Phenyl), $56.15\left(\mathrm{OCH}_{3}\right.$ Phenyl), $78.46(\mathrm{Cq} \mathrm{tBu}), 80.54(\mathrm{Cq} \mathrm{tBu})$, 112.32 (CH Phenyl), 114.22 (CH Phenyl), 115.83 (CH 6), 122.12 (CH 7), 122.82 (CH Phenyl), 123.26 (Cq 1), 125.75 (Cq 5a), 126.41 (CH 8), 127.07 (CH 9), 132.32 (CH 2), 138.24 (Cq 3a), 148.04 (Cq 4), 149.26 (Cq Phenyl), 150.10 (Cq Phenyl), 156.01 (Cq 9a), 172.36 (C=O). MS (ESI +, QTof, $m / z): 592.1[\mathrm{M}+\mathrm{H}]^{+}$. HRMS calculated for $\mathrm{C}_{32} \mathrm{H}_{42} \mathrm{~N}_{5} \mathrm{O}_{6}$ 592.3135, found 592.3137.

Tert-butyl 2-((1-(3,4-dihydroxyphenyl)imidazo[1,2-a]quinoxalin-4-yl)amino)acetate (9a): To a mixture of 7a $(0.320 \mathrm{~g}, 0.85 \mathrm{mmol})$ in $\mathrm{DME} / \mathrm{H}_{2} \mathrm{O}(2 / 1,15 \mathrm{~mL})$ were added compound 12 (0.392 g, $\left.2.54 \mathrm{mmol}\right)$, tetrakis(triphenylphosphine) palladium $(0.049 \mathrm{~g}, 0.040 \mathrm{mmol})$ and sodium carbonate $(0.179 \mathrm{~g}$, $1.70 \mathrm{mmol}$ ) in a microwave-adapted vial. The reaction was submitted to microwave irradiations during $20 \mathrm{~min}$ at $150{ }^{\circ} \mathrm{C}$ and then filtered on a Celite pad. The filtrate was concentrated under reduced pressure and successively washed with saturated aqueous ammonium chloride, saturated aqueous sodium bicarbonate, distilled water and finally brine. The organic phase was dried on sodium sulfate, filtered and concentrated under reduced pressure. The product was purified by flash chromatography eluted with cyclohexane/ethyl acetate $80 / 20$ to 50/50. The compound is obtained as a white solid (16\% yield). $\mathrm{C}_{22} \mathrm{H}_{22} \mathrm{~N}_{4} \mathrm{O}_{4}$. MW: $406.43 \mathrm{~g} / \mathrm{mol} .1 \mathrm{H}-\mathrm{NMR} \delta$ (ppm, DMSO- $d_{6}$ ): ${ }^{1} \mathrm{H}-\mathrm{NMR} \delta$ (ppm, DMSO- $d_{6}$ ): $1.42\left(\mathrm{~s}, 9 \mathrm{H}, 3 \times \mathrm{CH}_{3} \mathrm{OtBu}\right), 4.15\left(\mathrm{~d}, 2 \mathrm{H}, J=8 \mathrm{~Hz}, \mathrm{CH}_{2} \alpha\right), 6.82(\mathrm{dd}, 1 \mathrm{H}, J=4 \mathrm{~Hz}, J=8 \mathrm{~Hz}, \mathrm{CH}$ Phenyl), $6.89(\mathrm{dd}, 1 \mathrm{H}, J=4 \mathrm{~Hz}, J=8 \mathrm{~Hz}, \mathrm{CH}$ Phenyl), 6.93 (s, 1H, CH Phenyl), 7.02-7.06 (m, 1H, CH 7), 7.33 (dd, $1 \mathrm{H}, J=4 \mathrm{~Hz}, J=8 \mathrm{~Hz}, \mathrm{CH} 6), 7.35-7.38(\mathrm{~m}, 1 \mathrm{H}, \mathrm{CH} 8), 7.46(\mathrm{~s}, 1 \mathrm{H}, \mathrm{CH} 2), 7.54(\mathrm{dd}, 1 \mathrm{H}, J=4 \mathrm{~Hz}, J=8 \mathrm{~Hz}$, $\mathrm{CH}$ 9), 7.96-8.00 (m, 1H, NH), 9.32 (s, 1H, C-OH Phenyl), 9.41 (s, 1H, C-OH Phenyl). ${ }^{13} \mathrm{C}-\mathrm{NMR} \delta$ (ppm, DMSO- $\left.d_{6}\right): 28.23\left(\mathrm{CH}_{3} \mathrm{tBu}\right), 43.30\left(\mathrm{CH}_{2} \alpha\right), 80.85(\mathrm{Cq} \mathrm{tBu}), 115.37$ (Cq 1), $115.92(\mathrm{CH} 6), 116.48(\mathrm{CH}$ 7), 117.89 (CH Phenyl), 121.13 (CH Phenyl), 122.06 (CH 8), 122.81 (CH Phenyl), 125.98 (Cq 5a), 126.54 
(CH 9), 127.77 (Cq Phenyl), 131.43 (Cq 3a), 132.36 (CH 2), 132.89 (Cq 9a), 146.04 (Cq Phenyl), 147.13 (Cq Phenyl), 147.65 (Cq 4), 169.76 (C=O). MS (ESI +, QTof, $m / z): 407.2[\mathrm{M}+\mathrm{H}]^{+}$. HRMS calculated for $\mathrm{C}_{22} \mathrm{H}_{23} \mathrm{~N}_{4} \mathrm{O}_{4}$ 407.1706, found 407.1710.

Tert-butyl 2-((1-(3,4-dihydroxyphenyl)imidazo[1,2-a]quinoxalin-4-yl)amino)propanoate (9b): Following the same procedure used for the synthesis of $9 \mathbf{a}$ (see 4.1.4.8.), to a mixture of $7 \mathbf{b}(0.290 \mathrm{~g}, 0.74 \mathrm{mmol})$ in $\mathrm{DME} / \mathrm{H}_{2} \mathrm{O}(2 / 1,15 \mathrm{~mL})$ were added compound 12 (0.342 g, $\left.2.22 \mathrm{mmol}\right)$, tetrakis- (triphenylphosphine) palladium $(0.043 \mathrm{~g}, 0.037 \mathrm{mmol})$ and sodium carbonate $(0.157 \mathrm{~g}, 1.48 \mathrm{mmol})$ in a microwave-adapted vial. The product was purified by flash chromatography eluted with cyclohexane/ethyl acetate 80/20 to $50 / 50$. The compound is obtained as a white solid (6\% yield). $\mathrm{C}_{23} \mathrm{H}_{24} \mathrm{~N}_{4} \mathrm{O}_{4}$. MW: $420.46 \mathrm{~g} / \mathrm{mol}$. ${ }^{1} \mathrm{H}-\mathrm{NMR} \delta\left(\mathrm{ppm}, \mathrm{DMSO}-d_{6}\right): 1.42\left(\mathrm{~s}, 9 \mathrm{H}, 3 \times \mathrm{CH}_{3} \mathrm{OtBu}\right), 1.51\left(\mathrm{~d}, 3 \mathrm{H}, J=8 \mathrm{~Hz}, \mathrm{CH}_{3} \beta\right), 4.59-4.63(\mathrm{~m}$, $1 \mathrm{H}, \mathrm{CH} \alpha), 6.81(\mathrm{~d}, 1 \mathrm{H}, J=4 \mathrm{~Hz}, \mathrm{CH}$ Phenyl), 6.90 (d, 1H, J = 4 Hz, CH Phenyl), 6.93 (s, 1H, CH Phenyl), 7.01-7.05 (m, 1H, CH 7), $7.30(\mathrm{dd}, 1 \mathrm{H}, J=4 \mathrm{~Hz}, J=8 \mathrm{~Hz}, \mathrm{CH}$ 6), 7.33-7.37 (m, 1H, CH 8), $7.45(\mathrm{~s}, 1 \mathrm{H}$, CH 2), $7.53(\mathrm{dd}, 1 \mathrm{H}, J=4 \mathrm{~Hz}, J=8 \mathrm{~Hz}, \mathrm{CH}$ 9), $7.66(\mathrm{~d}, 1 \mathrm{H}, J=4 \mathrm{~Hz}, \mathrm{NH}), 9.36$ (s, 2H, C-OH Phenyl). ${ }^{13} \mathrm{C}-\mathrm{NMR} \delta$ (ppm, DMSO- $\left.d_{6}\right): 17.57\left(\mathrm{CH}_{3} \beta\right), 28.16\left(\mathrm{CH}_{3} \mathrm{tBu}\right), 50.17(\mathrm{CH} \alpha), 80.66(\mathrm{Cq} \mathrm{tBu}), 115.87(\mathrm{CH}$ 8), 116.47 (CH Phenyl), 117.88 (CH Phenyl), 121.16 (Cq 1), 122.05 (CH Phenyl), 122.78 (CH 7), 126.02 (Cq 5a), 127.16 (CH 9), 131.35 (Cq 3a), 132.19 (CH 2), 132.80 (Cq 9a), 137.57 (Cq 4), 146.03 (Cq Phenyl), 147.10 (Cq Phenyl), 147.23 (Cq Phenyl), 172.87 (C=O). MS (ESI +, QTof, $m / z): 421.2[\mathrm{M}+\mathrm{H}]^{+}$. HRMS calculated for $\mathrm{C}_{23} \mathrm{H}_{25} \mathrm{~N}_{4} \mathrm{O}_{4} 421.1876$, found 421.1875.

Tert-butyl 2-((1-(3,4-dihydroxyphenyl)imidazo[1,2-a]quinoxalin-4-yl)amino)-3-methyl-butanoate (9c): Following the same procedure used for the synthesis of $9 \mathrm{a}$ (see 4.1.4.8.), to a mixture of $7 \mathrm{c}(0.380 \mathrm{~g}$, $0.91 \mathrm{mmol})$ in $\mathrm{DME} / \mathrm{H}_{2} \mathrm{O}(2 / 1,15 \mathrm{~mL})$ were added compound $12(0.340 \mathrm{~g}, 2.72 \mathrm{mmol})$, tetrakis(triphenylphosphine) palladium $(0.052 \mathrm{~g}, 0.045 \mathrm{mmol})$ and sodium carbonate $(0.192 \mathrm{~g}, 1.81 \mathrm{mmol})$ in a microwave-adapted vial. The product was purified by flash chromatography eluted with cyclohexane/ethyl acetate $80 / 20$ to 50/50. The compound is obtained as a white solid (32\% yield). $\mathrm{C}_{25} \mathrm{H}_{28} \mathrm{~N}_{4} \mathrm{O}_{4}$. MW: $448.51 \mathrm{~g} / \mathrm{mol} .{ }^{1} \mathrm{H}-\mathrm{NMR} \delta$ (ppm, DMSO- $\left.d_{6}\right): 1.03\left(\mathrm{~d}, 3 \mathrm{H}, J=8 \mathrm{~Hz}, \mathrm{CH}_{3} \gamma\right), 1.06(\mathrm{~d}$, $\left.3 \mathrm{H}, J=8 \mathrm{~Hz}, \mathrm{CH}_{3} \gamma^{\prime}\right), 1.44\left(\mathrm{~s}, 9 \mathrm{H}, 3 \times \mathrm{CH}_{3} \mathrm{OtBu}\right), 2.31-2.35(\mathrm{~m}, 1 \mathrm{H}, \mathrm{CH} \beta), 4.53(\mathrm{t}, 1 \mathrm{H}, J=8 \mathrm{~Hz}, \mathrm{CH} \alpha)$, $6.81(\mathrm{dd}, 1 \mathrm{H}, J=4 \mathrm{~Hz}, J=8 \mathrm{~Hz}, \mathrm{CH}$ Phenyl), $6.90(\mathrm{~d}, 1 \mathrm{H}, J=4 \mathrm{~Hz}, J=8 \mathrm{~Hz}, \mathrm{CH}$ Phenyl), $6.93(\mathrm{~d}, 1 \mathrm{H}$, $J=4 \mathrm{~Hz}, J=8 \mathrm{~Hz}, \mathrm{CH}$ Phenyl), 7.03-7.05 (m, 1H, CH 7), 7.07 (d, 1H, $J=4 \mathrm{~Hz}, \mathrm{NH}), 7.34-7.35(\mathrm{~m}, 1 \mathrm{H}$, $\mathrm{CH} 8), 7.37(\mathrm{dd}, 1 \mathrm{H}, J=4 \mathrm{~Hz}, J=8 \mathrm{~Hz}, \mathrm{CH} 6), 7.46(\mathrm{~s}, 1 \mathrm{H}, \mathrm{CH} 2), 7.56(\mathrm{dd}, 1 \mathrm{H}, J=4 \mathrm{~Hz}, J=8 \mathrm{~Hz}, \mathrm{CH} 9)$, 9.32 (s, $1 \mathrm{H}, \mathrm{C}-\mathrm{OH}$ Phenyl), 9.41 (s, $1 \mathrm{H}, \mathrm{C}-\mathrm{OH}$ Phenyl). ${ }^{13} \mathrm{C}-\mathrm{NMR} \delta$ (ppm, DMSO- $\left.d_{6}\right): 19.30\left(\mathrm{CH}_{3} \gamma^{\prime}\right)$, $19.47\left(\mathrm{CH}_{3} \gamma\right), 28.19\left(\mathrm{CH}_{3} \mathrm{tBu}\right), 30.54(\mathrm{CH} \beta), 59.60(\mathrm{CH} \alpha), 81.25(\mathrm{Cq} \mathrm{tBu}), 115.90(\mathrm{CH} 6), 116.47(\mathrm{CH}$ Phenyl), 117.89 (CH Phenyl), 121.05 (Cq 1), 122.07 (CH Phenyl), 123.07 (CH 7), 126.11 (Cq 5a), 126.56 (CH 8), 127.28 (CH 9), 131.55 (Cq Phenyl), 132.24 (CH 2), 132.72 (Cq 3a), 137.43 (Cq 9a), 146.03 (Cq 4), 147.14 (Cq Phenyl), 147.47 (Cq Phenyl), 171.62 (C=O). MS (ESI +, QTof, $m / z): 449.3[\mathrm{M}+\mathrm{H}]^{+}$. HRMS calculated for $\mathrm{C}_{25} \mathrm{H}_{29} \mathrm{~N}_{4} \mathrm{O}_{4} 449.2189$, found 449.2188 .

Tert-butyl 2-((1-(3,4-dihydroxyphenyl)imidazo[1,2-a]quinoxalin-4-yl)amino)-4-methyl-pentanoate (9d): Following the same procedure used for the synthesis of $9 \mathrm{a}$ (see 4.1.4.8.), to a mixture of $7 \mathrm{~d}(0.460 \mathrm{~g}$, $1.06 \mathrm{mmol})$ in $\mathrm{DME} / \mathrm{H}_{2} \mathrm{O}(2 / 1,15 \mathrm{~mL})$ were added compound $12(0.400 \mathrm{~g}, 2.6 \mathrm{mmol})$, tetrakis(triphenylphosphine) palladium $(0.062 \mathrm{~g}, 0.053 \mathrm{mmol})$ and sodium carbonate $(0.224 \mathrm{~g}, 2.11 \mathrm{mmol})$ in a microwave-adapted vial. The product was purified by flash chromatography eluted with cyclohexane/ethyl acetate $80 / 20$ to 50/50. The compound is obtained as a white solid (16\% yield). $\mathrm{C}_{26} \mathrm{H}_{30} \mathrm{~N}_{4} \mathrm{O}_{4}$. MW: $462.54 \mathrm{~g} / \mathrm{mol} .{ }^{1} \mathrm{H}-\mathrm{NMR} \delta$ (ppm, DMSO- $\left.d_{6}\right): 0.93\left(\mathrm{~d}, 3 \mathrm{H}, J=8 \mathrm{~Hz}, \mathrm{CH}_{3} \delta\right), 0.97(\mathrm{~d}$, $\left.3 \mathrm{H}, J=8 \mathrm{~Hz}, \mathrm{CH}_{3} \delta^{\prime}\right), 1.42\left(\mathrm{~s}, 9 \mathrm{H}, 3 \times \mathrm{CH}_{3} \mathrm{OtBu}\right), 1.56-1.60\left(\mathrm{~m}, 1 \mathrm{H}, \mathrm{CH}_{2} \beta\right), 1.79-1.83(\mathrm{~m}, 1 \mathrm{H}, \mathrm{CH} \gamma)$, 1.91-1.95 (m, 1H, CH $\left.\mathrm{CH}_{2} \beta\right), 4.65(\mathrm{t}, 1 \mathrm{H}, J=4 \mathrm{~Hz}, \mathrm{CH} \alpha), 6.81(\mathrm{~d}, 1 \mathrm{H}, J=4 \mathrm{~Hz}, \mathrm{CH}$ Phenyl), $6.86(\mathrm{~s}, 1 \mathrm{H}$, CH Phenyl), $6.90(\mathrm{~d}, 1 \mathrm{H}, J=4 \mathrm{~Hz}, \mathrm{CH}$ Phenyl), 7.01-7.05 (m, 1H, CH 7), 7.28-7.32 (m, 1H, CH 8), 7.34 $(\mathrm{dd}, 1 \mathrm{H}, J=4 \mathrm{~Hz}, J=8 \mathrm{~Hz}, \mathrm{CH} 6), 7.46(\mathrm{~s}, 1 \mathrm{H}, \mathrm{CH} 2), 7.55(\mathrm{dd}, 1 \mathrm{H}, J=4 \mathrm{~Hz}, J=8 \mathrm{~Hz}, \mathrm{CH}$ 9), $7.59(\mathrm{~d}, 1 \mathrm{H}$, $J=4 \mathrm{~Hz}, \mathrm{NH}), 9.38$ (s, 2H, C-OH Phenyl). ${ }^{13} \mathrm{C}-\mathrm{NMR} \delta$ (ppm, DMSO-d $\left.d_{6}\right): 22.09\left(\mathrm{CH}_{3} \delta\right), 23.32\left(\mathrm{CH}_{3}\right.$ $\left.\delta^{\prime}\right), 25.17(\mathrm{CH} \gamma), 28.18\left(\mathrm{CH}_{3} \mathrm{tBu}\right), 39.60\left(\mathrm{CH}_{2} \beta\right), 52.88(\mathrm{CH} \alpha), 80.76(\mathrm{Cq} \mathrm{tBu}), 115.87(\mathrm{CH} 6), 116.48$ (CH Phenyl), 117.90 (CH Phenyl), 121.15 (Cq 1), 122.18 (CH Phenyl), 122.79 (CH 7), 126.11 (Cq 5a), 
125.80 (CH 8), 126.04 (CH 9), 131.42 (Cq 3a), 132.18 (CH 2), 137.58 (Cq 9a), 146.06 (Cq 4), 147.14 (Cq Phenyl), 147.57 (Cq Phenyl), $172.77(\mathrm{C}=\mathrm{O})$. MS (ESI +, QTof, $m / z): 463.2[\mathrm{M}+\mathrm{H}]^{+}$. HRMS calculated for $\mathrm{C}_{26} \mathrm{H}_{31} \mathrm{~N}_{4} \mathrm{O}_{4} 463.2345$, found 463.2343 .

Tert-butyl 6-((Tert-butoxycarbonyl)amino)-2-((1-(3,4-dihydroxyphenyl)imidazo[1,2-a]-quinoxalin-4-yl)amino) hexanoate (9e): Following the same procedure used for the synthesis of $9 \mathbf{a}$ (see 4.1.4.8.), to a mixture of $7 \mathbf{e}(0.242 \mathrm{~g}, 0.44 \mathrm{mmol})$ in $\mathrm{DME} / \mathrm{H}_{2} \mathrm{O}(2 / 1,15 \mathrm{~mL})$ were added compound $12(0.170 \mathrm{~g}, 1.10 \mathrm{mmol})$, tetrakis- (triphenylphosphine) palladium $(0.025 \mathrm{~g}, 0.022 \mathrm{mmol})$ and sodium carbonate $(0.093 \mathrm{~g}$, $0.88 \mathrm{mmol}$ ) in a microwave-adapted vial. The product was purified by flash chromatography eluted with cyclohexane/ethyl acetate $80 / 20$ to $50 / 50$. The compound is obtained as a white solid $(40 \%$ yield). $\mathrm{C}_{31} \mathrm{H}_{39} \mathrm{~N}_{5} \mathrm{O}_{6}$. MW: $577.67 \mathrm{~g} / \mathrm{mol} .{ }^{1} \mathrm{H}-\mathrm{NMR} \delta$ (ppm, DMSO- $\left.d_{6}\right): 1.35$ (s, 9H, $3 \times \mathrm{CH}_{3} \mathrm{OtBu}$ ), 1.40-1.42 (m, 2H, CH $\left.\mathrm{CH}_{2} \gamma\right), 1.43\left(\mathrm{~s}, 9 \mathrm{H}, 3 \times \mathrm{CH}_{3} \mathrm{OtBu}\right), 1.45-1.47\left(\mathrm{~m}, 2 \mathrm{H}, \mathrm{CH}_{2} \delta\right), 1.88-2.00\left(\mathrm{~m}, 2 \mathrm{H}, \mathrm{CH}_{2}\right.$ ß), 2.92-2.94 (m, 2H, $\left.\mathrm{CH}_{2} \varepsilon\right), 4.55-4.56(\mathrm{~m}, 1 \mathrm{H}, \mathrm{CH} \alpha), 6.79\left(\mathrm{t}, 1 \mathrm{H}, J=4 \mathrm{~Hz}, \mathrm{NH}-\mathrm{CH}_{2} \varepsilon\right), 6.83(\mathrm{~d}, 1 \mathrm{H}$, $J=4 \mathrm{~Hz}, \mathrm{CH}$ Phenyl), 6.89 (s, 1H, CH Phenyl), 6.93 (d, 1H, J = 4 Hz, CH Phenyl), 7.02-7.05 (m, 1H, CH 7), 7.31-7.33 (m, 1H, CH 8), $7.36(\mathrm{dd}, 1 \mathrm{H}, J=4 \mathrm{~Hz}, J=8 \mathrm{~Hz}, \mathrm{CH} 6), 7.45$ (s, $1 \mathrm{H}, \mathrm{CH} 2), 7.54(\mathrm{dd}, 1 \mathrm{H}, J=4$ $\mathrm{Hz}, J=8 \mathrm{~Hz}, \mathrm{CH}$ 9), 7.57 (d, 1H, J = 4 Hz, NH-CH $\alpha$ ), 9.33 (s, 1H, C-OH Phenyl), 9.41 (s, 1H, C-OH Phenyl). ${ }^{13} \mathrm{C}-\mathrm{NMR} \delta$ (ppm, DMSO- $\left.d_{6}\right): 22.40\left(\mathrm{CH}_{2} \gamma\right), 27.11\left(\mathrm{CH}_{3} \mathrm{tBu}\right), 27.65\left(\mathrm{CH}_{3} \mathrm{tBu}\right), 28.56\left(\mathrm{CH}_{2} \delta\right)$, $29.91\left(\mathrm{CH}_{2} \beta\right), 39.25\left(\mathrm{CH}_{2} \varepsilon\right), 53.44(\mathrm{CH} \alpha), 76.70(\mathrm{Cq} \mathrm{tBu}), 79.75(\mathrm{Cq} \mathrm{tBu}), 114.79(\mathrm{CH}), 115.40(\mathrm{CH}$ Phenyl), 116.80 (CH Phenyl), 120.07 (CH Phenyl), 121.73 (CH 7), 124.97 (Cq 5a), 125.42 (CH 8), 126.12 (CH 9), 130.32 (Cq 1), 131.09 (CH 2), 131.70 (Cq 3a), 136.49 (Cq 9a), 144.96 (Cq 4), 146.04 (Cq Phenyl), 146.43 (Cq Phenyl), 154.96 (Cq Phenyl), 172.41 (C=O). MS (ESI +, QTof, $m / z): 578.3[\mathrm{M}+\mathrm{H}]^{+}$. HRMS calculated for $\mathrm{C}_{31} \mathrm{H}_{40} \mathrm{~N}_{5} \mathrm{O}_{6} 578.2979$, found 578.2980.

Tert-butyl 5-(((benzyloxy)carbonyl)amino)-2-((1-(3,4-dihydroxyphenyl)imidazo[1,2-a]-quinoxalin-4-yl)amino) pentanoate (9f): Following the same procedure used for the synthesis of $9 \mathbf{a}$ (see 4.1.4.8.), to a mixture of $7 \mathfrak{f}(0.260 \mathrm{~g}, 0.46 \mathrm{mmol})$ in $\mathrm{DME} / \mathrm{H}_{2} \mathrm{O}(2 / 1,15 \mathrm{~mL})$ were added compound $12(0.211 \mathrm{~g}, 1.37 \mathrm{mmol})$, tetrakis- (triphenylphosphine) palladium $(0.026 \mathrm{~g}, 0.023 \mathrm{mmol})$ and sodium carbonate $(0.097 \mathrm{~g}$, $0.91 \mathrm{mmol}$ ) in a microwave-adapted vial. The product was purified by flash chromatography eluted with cyclohexane/ethyl acetate $80 / 20$ to $50 / 50$. The compound is obtained as a white solid $(26 \%$ yield). $\mathrm{C}_{33} \mathrm{H}_{35} \mathrm{~N}_{5} \mathrm{O}_{6}$. MW: $597.66 \mathrm{~g} / \mathrm{mol} .{ }^{1} \mathrm{H}-\mathrm{NMR} \delta$ (ppm, DMSO- $d_{6}$ ): ${ }^{1} \mathrm{H}-\mathrm{NMR} \delta(\mathrm{ppm}, 400 \mathrm{MHz}$, DMSO- $\left.d_{6}\right): 1.40\left(\mathrm{~s}, 9 \mathrm{H}, 3 \times \mathrm{CH}_{3} \mathrm{OtBu}\right), 1.58-1.60\left(\mathrm{~m}, 2 \mathrm{H}, \mathrm{CH}_{2} \gamma\right), 1.91-1.95\left(\mathrm{~m}, 2 \mathrm{H}, \mathrm{CH}_{2} \beta\right), 3.05-3.08$ $\left(\mathrm{m}, 2 \mathrm{H}, \mathrm{CH}_{2} \delta\right), 4.55-4.59(\mathrm{~m}, 1 \mathrm{H}, \mathrm{CH} \alpha), 5.01\left(\mathrm{~s}, 2 \mathrm{H}, \mathrm{CH}_{2}\right.$-Phenyl), $6.81(\mathrm{~d}, 1 \mathrm{H}, J=4 \mathrm{~Hz}, \mathrm{CH}$ Phenyl), 6.89 (s, 1H, CH Phenyl), 6.93 (d, 1H, J = 4 Hz, CH Phenyl), 7.01-7.06 (m, 1H, CH 7), 7.27 (d, 1H, J = 4 $\left.\mathrm{Hz}, \mathrm{NH}-\mathrm{CH}_{2} \delta\right), 7.31-7.34$ (m, 5H, CH Phenyl), 7.37 (dd, $\left.1 \mathrm{H}, J=4 \mathrm{~Hz}, J=8 \mathrm{~Hz}, \mathrm{CH} 6\right), 7.45(\mathrm{~s}, 1 \mathrm{H}, \mathrm{CH}$ 2), $7.54(\mathrm{~d}, 1 \mathrm{H}, J=4 \mathrm{~Hz}, \mathrm{NH}-\mathrm{CH} \alpha), 7.56-7.60(\mathrm{~m}, 1 \mathrm{H}, \mathrm{CH} 8), 7.63(\mathrm{dd}, 1 \mathrm{H}, \mathrm{J}=J=4 \mathrm{~Hz}, J=8 \mathrm{~Hz}, \mathrm{CH} 9)$. ${ }^{13} \mathrm{C}-\mathrm{NMR} \delta$ (ppm, DMSO- $\left.d_{6}\right): 26.54\left(\mathrm{CH}_{2} \gamma\right), 28.09\left(\mathrm{CH}_{2} \beta\right), 28.17\left(\mathrm{CH}_{3} \mathrm{tBu}\right), 40.45\left(\mathrm{CH}_{2} \delta\right), 54.34(\mathrm{CH}$ $\alpha), 65.60$ ( $\mathrm{CH}_{2}$-Phenyl), 80.92 (Cq tBu), 115.88 (CH 6), 116.48 (CH Phenyl), 117.90 (CH Phenyl), 121.16 (Cq 1), 122.84 (CH 7), 126.06 (Cq 5a), 126.50 (CH 9), 127.20 (CH 8), 128.18 (CH Phenyl), 128.79 (CH Phenyl), 129.16 (CH Phenyl), 131.90 (CH 2), 132.79 (Cq 3a), 137.55 (Cq 9a), 146.04 (Cq 4), 147.12 (Cq Phenyl), 147.47 (Cq Phenyl), 156.59 (Cq Phenyl), 172.23 (C=O). MS (ESI +, QTof, $m / z)$ : $598.3[\mathrm{M}+\mathrm{H}]^{+}$. HRMS calculated for $\mathrm{C}_{33} \mathrm{H}_{36} \mathrm{~N}_{5} \mathrm{O}_{6}$ 598.2666, found 598.2670.

Tert-butyl 2-((1-(3,4-dihydroxyphenyl)imidazo[1,2-a]quinoxalin-4-yl)amino)-3-phenyl-propanoate (9g): Following the same procedure used for the synthesis of $9 \mathrm{a}$ (see 4.1.4.8.), to a mixture of $7 \mathrm{~g}(0.360 \mathrm{~g}$, $0.77 \mathrm{mmol})$ in DME $/ \mathrm{H}_{2} \mathrm{O}(2 / 1,15 \mathrm{~mL})$ were added compound $12(0.356 \mathrm{~g}, 2.31 \mathrm{mmol})$, tetrakis(triphenylphosphine) palladium $(0.044 \mathrm{~g}, 0.038 \mathrm{mmol})$ and sodium carbonate $(0.163 \mathrm{~g}, 1.54 \mathrm{mmol})$ in a microwave-adapted vial. The product was purified by flash chromatography eluted with cyclohexane/ethyl acetate $80 / 20$ to $50 / 50$. The compound is obtained as a white solid (16\% yield). $\mathrm{C}_{29} \mathrm{H}_{28} \mathrm{~N}_{4} \mathrm{O}_{4}$. MW: $496.56 \mathrm{~g} / \mathrm{mol} .{ }^{1} \mathrm{H}-\mathrm{NMR} \delta$ (ppm, DMSO- $d_{6}$ ): 1.36 (s, 9H, $3 \times \mathrm{CH}_{3} \mathrm{OtBu}$ ), 3.23-3.27 $\left(\mathrm{m}, 1 \mathrm{H}, \mathrm{CH}_{2} \beta\right), 3.33-3.38\left(\mathrm{~m}, 1 \mathrm{H}, \mathrm{CH}_{2} \beta\right), 4.91-4.93(\mathrm{~m}, 1 \mathrm{H}, \mathrm{CH} \alpha), 6.81(\mathrm{dd}, 1 \mathrm{H}, J=4 \mathrm{~Hz}, J=8 \mathrm{~Hz}, \mathrm{CH}$ Phenyl), 6.89 (d, 1H, J = 4 Hz, CH Phenyl), $6.92(\mathrm{~d}, 1 \mathrm{H}, J=8 \mathrm{~Hz}, \mathrm{CH}$ Phenyl), 7.08 (t, 1H, $J=8 \mathrm{~Hz}, \mathrm{CH}$ Phenyl), 7.20 (t, 1H, $J=8 \mathrm{~Hz}, \mathrm{CH}$ Phenyl), $7.29(\mathrm{t}, 2 \mathrm{H}, J=8 \mathrm{~Hz}, \mathrm{CH}$ Phenyl), $7.34(\mathrm{~d}, 2 \mathrm{H}, J=8 \mathrm{~Hz}, \mathrm{CH}$, 
CH Phenyl), 7.34-7.37 (m, 1H, CH 7), 7.38-7.40 (m, 1H, CH 8), 7.52 (s, 1H, CH 2), 7.60 (d, 1H, J = 8 $\mathrm{Hz}, \mathrm{CH}$ 9), 7.92-7.93 (m, 1H, NH), 9.38 (s, 2H, C-OH Phenyl). ${ }^{13} \mathrm{C}-\mathrm{NMR} \delta$ (ppm, DMSO- $d_{6}$ ): 26.98 $\left(\mathrm{CH}_{3} \mathrm{tBu}\right), 35.93\left(\mathrm{CH}_{2} \beta\right), 55.04(\mathrm{CH} \alpha), 80.23(\mathrm{Cq} \mathrm{tBu}), 114.96(\mathrm{CH} 6), 115.42(\mathrm{CH}$ Phenyl), $116.75(\mathrm{CH}$ Phenyl), 119.67 (Cq 1), 120.96 (CH Phenyl), 122.32 (CH 7), 124.69 (Cq 5a), 125.73 (CH Phenyl, CH 9), 125.94 (CH Phenyl), 127.90 (CH Phenyl), 128.71 (CH 8), 130.76 (Cq Phenyl), 131.11 (CH 2), 131.21 (Cq Phenyl), 136.87 (Cq 3a), 138.12 (Cq 9a), 146.17 (Cq 4), 170.13 (C=O). MS (ESI +, QTof, m/z): 497.1 [M + $\mathrm{H}]^{+}$. HRMS calculated for $\mathrm{C}_{29} \mathrm{H}_{29} \mathrm{~N}_{4} \mathrm{O}_{4} 497.2189$, found 497.2198.

Tert-butyl 3-(4-(Tert-butoxy)phenyl)-2-((1-(3,4-dihydroxyphenyl)imidazo[1,2-a]quinoxalin-4-yl)amino) propanoate (9h): Following the same procedure used for the synthesis of $9 \mathrm{a}$ (see 4.1.4.8.), to a mixture of 7h $(0.260 \mathrm{~g}, 0.57 \mathrm{mmol})$ in $\mathrm{DME} / \mathrm{H}_{2} \mathrm{O}(2 / 1,15 \mathrm{~mL})$ were added compound $12(0.170 \mathrm{~g}, 1.72 \mathrm{mmol})$, tetrakis- (triphenylphosphine) palladium $(0.028 \mathrm{~g}, 0.028 \mathrm{mmol})$ and sodium carbonate $(0.101 \mathrm{~g}$, $1.15 \mathrm{mmol}$ ) in a microwave-adapted vial. The product was purified by flash chromatography eluted with cyclohexane/ethyl acetate $80 / 20$ to $50 / 50$. The compound is obtained as a white solid ( $22 \%$ yield). $\mathrm{C}_{33} \mathrm{H}_{36} \mathrm{~N}_{4} \mathrm{O}_{5}$. MW: $568.66 \mathrm{~g} / \mathrm{mol} .{ }^{1} \mathrm{H}-\mathrm{NMR} \delta$ (ppm, DMSO- $d_{6}$ ): 1.24 (s, 9H, $3 \times \mathrm{CH}_{3} \mathrm{OtBu}$ ), $1.33\left(\mathrm{~s}, 9 \mathrm{H}, 3 \times \mathrm{CH}_{3} \mathrm{OtBu}\right), 3.12-3.18\left(\mathrm{~m}, 1 \mathrm{H}, \mathrm{CH}_{2} \beta\right), 3.26-3.32\left(\mathrm{~m}, 1 \mathrm{H}, \mathrm{CH}_{2} \beta\right), 4.80-4.84(\mathrm{~m}, 1 \mathrm{H}$, $\mathrm{CH} \alpha), 6.80(\mathrm{~d}, 1 \mathrm{H}, J=8 \mathrm{~Hz}, \mathrm{CH}$ Phenyl), $6.88(\mathrm{~s}, 1 \mathrm{H}, \mathrm{CH}$ Phenyl), $6.90(\mathrm{dd}, 2 \mathrm{H}, J=4 \mathrm{~Hz}, J=8 \mathrm{~Hz}$, CH Phenyl), 6.92 (d, 1H, J = 8 Hz, CH Phenyl), 7.01-7.05 (m, 1H, CH 7), 7.25 (d, 2H, J = 8 Hz, CH Phenyl), 7.30-7.32 (m, 1H, CH 8), $7.34(\mathrm{dd}, 1 \mathrm{H}, J=4 \mathrm{~Hz}, J=8 \mathrm{~Hz}, \mathrm{CH}$ 6), 7.45 (s, 1H, CH 2), 7.54 (dd, $1 \mathrm{H}, J=4 \mathrm{~Hz}, J=8 \mathrm{~Hz}, \mathrm{CH}$ 9), 7.57 (d, 1H, $J=8 \mathrm{~Hz}, \mathrm{NH}$ ), 9.31 (s, 1H, C-OH Phenyl), 9.40 (s, 1H, C-OH Phenyl). ${ }^{13} \mathrm{C}-\mathrm{NMR} \delta$ (ppm, DMSO- $\left.d_{6}\right): 28.05\left(\mathrm{CH}_{3} \mathrm{tBu}\right), 28.95\left(\mathrm{CH}_{3} \mathrm{tBu}\right), 36.64\left(\mathrm{CH}_{2} \beta\right), 55.87(\mathrm{CH} \alpha)$, 78.12 (Cq tBu), 80.91 (Cq tBu), 115.90 (CH 6), 116.46 (CH Phenyl), 117.87 (CH Phenyl), 121.08 (Cq 1), 122.05 (CH Phenyl), 122.91 (CH 7), 124.00 (2 × CH Phenyl), 126.07 (Cq 5a), 126.49 (CH 8), 127.21 (CH 9), 130.33 ( $2 \times \mathrm{CH}$ Phenyl), 132.26 (CH 2), 132.62 (Cq 3a), 137.46 (Cq 9a), 146.02 (Cq 4), 147.11 (Cq Phenyl), 147.22 (Cq Phenyl), 154.05 ( $2 \times$ Cq Phenyl), 171.75 (C=O). MS (ESI +, QTof, $m / z): 569.3$ [M + $\mathrm{H}]^{+}$. HRMS calculated for $\mathrm{C}_{33} \mathrm{H}_{37} \mathrm{~N}_{4} \mathrm{O}_{5} 569.2764$, found 569.2773.

Tert-butyl 2-((Tert-butoxycarbonyl)amino)-5-((1-(3,4-dihydroxyphenyl)imidazo[1,2-a]-quinoxalin-4-yl)amino) pentanoate (9i): Following the same procedure used for the synthesis of $9 a$ (see 4.1.4.8.), to a mixture of $7 \mathbf{i}(0.460 \mathrm{~g}, 0.86 \mathrm{mmol})$ in $\mathrm{DME} / \mathrm{H}_{2} \mathrm{O}(2 / 1,15 \mathrm{~mL})$ were added compound $\mathbf{1 2}(0.330 \mathrm{~g}, 2.15 \mathrm{mmol})$, tetrakis- (triphenylphosphine) palladium $(0.050 \mathrm{~g}, 0.043 \mathrm{mmol})$ and sodium carbonate $(0.182 \mathrm{~g}$, $1.72 \mathrm{mmol}$ ) in a microwave-adapted vial. The product was purified by flash chromatography eluted with cyclohexane/ethyl acetate $80 / 20$ to $50 / 50$. The compound is obtained as a white solid (30\% yield). $\mathrm{C}_{30} \mathrm{H}_{37} \mathrm{~N}_{5} \mathrm{O}_{6}$. MW: $563.64 \mathrm{~g} / \mathrm{mol} .{ }^{1} \mathrm{H}-\mathrm{NMR} \delta$ (ppm, DMSO- $d_{6}$ ): 1.33 (s, 9H, CH-COOtBu), 1.39 (s, 9H, NH-COOtBu), 1.66-1.72 (m, 4H, $\left.\mathrm{CH}_{2} \beta, \mathrm{CH}_{2} \gamma\right), 3.56-3.57\left(\mathrm{~m}, 2 \mathrm{H}, \mathrm{CH}_{2} \delta\right), 3.89-3.93(\mathrm{~m}, 1 \mathrm{H}, \mathrm{CH} \alpha)$, $6.79(\mathrm{dd}, 1 \mathrm{H}, J=4 \mathrm{~Hz}, J=8 \mathrm{~Hz}, \mathrm{CH}$ Phenyl), $6.87(\mathrm{~d}, 1 \mathrm{H}, J=4 \mathrm{~Hz}, \mathrm{CH}$ Phenyl), $6.90(\mathrm{~d}, 1 \mathrm{H}, J=8 \mathrm{~Hz}$, CH Phenyl), 6.95-7.00 (m, 1H, CH 7), 7.17 (d, 1H, J = 8 Hz, NH-CH $\alpha), 7.27-7.29$ (m, 1H, CH 8), 7.31 $(\mathrm{dd}, 1 \mathrm{H}, J=4 \mathrm{~Hz}, J=8 \mathrm{~Hz}, \mathrm{CH} 6), 7.40(\mathrm{~s}, 1 \mathrm{H}, \mathrm{CH} 2), 7.56(\mathrm{dd}, 1 \mathrm{H}, J=4 \mathrm{~Hz}, J=8 \mathrm{~Hz}, \mathrm{CH} 9), 7.69(\mathrm{t}, 1 \mathrm{H}$, $\left.J=4 \mathrm{~Hz}, \mathrm{NH}-\mathrm{CH}_{2} \delta\right), 9.35$ (s, 2H, C-OH Phenyl). ${ }^{13} \mathrm{C}-\mathrm{NMR} \delta$ (ppm, DMSO- $\left.d_{6}\right): 26.80\left(\mathrm{CH}_{2} \beta\right), 28.04$ $\left(\mathrm{CH}_{3} \mathrm{tBu}\right), 28.67\left(\mathrm{CH}_{3} \mathrm{tBu}\right), 30.42\left(\mathrm{CH}_{2} \gamma\right), 39.79\left(\mathrm{CH}_{2} \delta\right), 54.78(\mathrm{CH} \alpha), 78.46(\mathrm{Cq} \mathrm{tBu}), 80.53(\mathrm{Cq} \mathrm{tBu})$, 115.79 (CH 6), 116.45 (CH Phenyl), 117.86 (CH Phenyl), 121.29 (Cq 1), 122.01 (CH Phenyl), 122.06 (CH 7), 125.76 (Cq 5a), 126.29 (CH 8), 127.03 (CH 9), 131.91 (CH 2), 138.20 (Cq 3a), 146.02 (Cq 4), 147.05 (Cq Phenyl), 148.03 (Cq Phenyl), 156.02 (Cq 9a), 172.34 (C=O). MS (ESI +, QTof, $m / z): 564.3[\mathrm{M}+\mathrm{H}]^{+}$. HRMS calculated for $\mathrm{C}_{30} \mathrm{H}_{38} \mathrm{~N}_{5} \mathrm{O}_{6} 564.2822$, found 564.2827.

\subsubsection{Cleavage of the Protective Groups}

2-((1-(3,4-Dimethoxyphenyl)imidazo[1,2-a]quinoxalin-4-yl)amino)propanoic acid $(\mathbf{1 0 b})$ : To a cooled $\left(0{ }^{\circ} \mathrm{C}\right)$ solution of $8 \mathbf{b}(0.170 \mathrm{~g}, 0.36 \mathrm{mmol})$ in anhydrous $\mathrm{CH}_{2} \mathrm{Cl}_{2}(20 \mathrm{~mL})$ was added boron tribromide $(2.1 \mathrm{~mL}$, $2.1 \mathrm{mmol})$. The resulting solution was allowed to warm to room temperature and stirred until complete consumption of the starting material (1-3 h, monitored by TLC). The solution was neutralized by addition of saturated aqueous sodium bicarbonate $(20 \mathrm{~mL})$. The crude mixture was extracted with 
$\mathrm{CH}_{2} \mathrm{Cl}_{2}(3 \times 20 \mathrm{~mL})$. The organic phase was dried on sodium sulfate, filtered and concentrated under reduced pressure. The product was purified by flash chromatography eluted with cyclohexane/ethyl acetate $90 / 20$ to $40 / 50$. The compound is obtained as a white solid (71\% yield). $\mathrm{C}_{21} \mathrm{H}_{20} \mathrm{~N}_{4} \mathrm{O}_{4}$. $\mathrm{MW}$ $392.41 \mathrm{~g} / \mathrm{mol} .{ }^{1} \mathrm{H}-\mathrm{NMR} \delta$ (ppm, DMSO- $\left.d_{6}\right): 1.24$ (s, $\left.1 \mathrm{H}, \mathrm{COOH}\right), 1.53$ (d, 3H, J = 8 Hz, $\mathrm{CH}_{3} \beta$ ), 3.75 (s, $3 \mathrm{H}, \mathrm{OCH}_{3}$ Phenyl), 3.87 (s, 3H, OCH 3 Phenyl), 4.61-4.63 (m, 1H, CH $\left.\alpha\right), 7.00-7.04$ (m, 1H, CH 7), 7.11 (d, 1H, J = 4 Hz, CH Phenyl), 7.13 (s, 1H, CH Phenyl), 7.17 (d, 1H, J = 4 Hz, CH Phenyl), 7.31 (dd, 1H, $J=4 \mathrm{~Hz}, J=8 \mathrm{~Hz}, \mathrm{CH}$ 6), 7.33-7.35 (m, 1H, CH 8), 7.50 (s, 1H, CH 2), 7.52 (d, 1H, J = $4 \mathrm{~Hz}, \mathrm{NH}), 7.60$ $(\mathrm{dd}, 1 \mathrm{H}, J=4 \mathrm{~Hz}, J=8 \mathrm{~Hz}, \mathrm{CH} 9) .{ }^{13} \mathrm{C}-\mathrm{NMR} \delta\left(\mathrm{ppm}, \mathrm{DMSO}-d_{6}\right): 18.71\left(\mathrm{CH}_{3} \beta\right), 50.30(\mathrm{CH} \alpha), 56.07$ $\left(\mathrm{OCH}_{3}\right.$ Phenyl), $56.16\left(\mathrm{OCH}_{3}\right.$ Phenyl), 112.31 (CH Phenyl), 114.22 (CH Phenyl), 115.92 (CH 6), 122.54 (CH 7), 122.70 (Cq 1), 123.28 (CH Phenyl), 126.54 (CH 8), 125.90 (Cq 5a), 127.21 (CH 9), 130.95 (Cq 3a), 132.63 (CH 2), 137.99 (Cq 9a), 147.05 (Cq 4), 149.26 (Cq Phenyl), 150.132 (Cq Phenyl), 172.96 (C=O). MS $\left(\right.$ ESI +, QTof, m/z): $393.0[\mathrm{M}+\mathrm{H}]^{+}$. HRMS calculated for $\mathrm{C}_{21} \mathrm{H}_{21} \mathrm{~N}_{4} \mathrm{O}_{4}$ 393.1563, found 393.1558.

2-((1-(3,4-Dimethoxyphenyl)imidazo[1,2-a]quinoxalin-4-yl)amino)-3-methylbutanoic acid (10c): Following the same procedure used for the synthesis of $10 \mathrm{~b}$, to a cooled solution of $8 \mathrm{c}(0.290 \mathrm{~g}, 0.61 \mathrm{mmol})$ in anhydrous $\mathrm{CH}_{2} \mathrm{Cl}_{2}(20 \mathrm{~mL})$ was added boron tribromide $(3.6 \mathrm{~mL}, 3.6 \mathrm{mmol})$. The product was purified by flash chromatography eluted with cyclohexane/ethyl acetate $90 / 20$ to $40 / 50$. The compound is obtained as a white solid (80\% yield). $\mathrm{C}_{23} \mathrm{H}_{24} \mathrm{~N}_{4} \mathrm{O}_{4}$. MW: $420.46 \mathrm{~g} / \mathrm{mol} .{ }^{1} \mathrm{H}-\mathrm{NMR} \delta$ (ppm, DMSO- $\left.d_{6}\right)$ : $0.99\left(\mathrm{~d}, 3 \mathrm{H}, \mathrm{J}=8 \mathrm{~Hz}, \mathrm{CH}_{3} \gamma\right), 1.01\left(\mathrm{~d}, 3 \mathrm{H}, \mathrm{J}=8 \mathrm{~Hz}, \mathrm{CH}_{3} \gamma^{\prime}\right), 1.23(\mathrm{~s}, 1 \mathrm{H}, \mathrm{COOH}), 2.36-2.41(\mathrm{~m}, 1 \mathrm{H}, \mathrm{CH}$ ß), 3.74 (s, 3H, $\mathrm{OCH}_{3}$ Phenyl), $3.87\left(\mathrm{~s}, 3 \mathrm{H}, \mathrm{OCH}_{3}\right.$ Phenyl), $4.55(\mathrm{t}, 1 \mathrm{H}, \mathrm{J}=16 \mathrm{~Hz}, \mathrm{CH} \alpha), 6.97-7.01(\mathrm{~m}$, 1H, CH 7), 7.10 (dd, 1H, J = 4 Hz, J = 8 Hz, CH Phenyl), 7.14 (s, 1H, CH Phenyl), 7.16 (dd, 1H, J = $4 \mathrm{~Hz}$, $J=8 \mathrm{~Hz}, \mathrm{CH}$ Phenyl), $7.19(\mathrm{~d}, 1 \mathrm{H}, \mathrm{NH}-\mathrm{CH} \alpha), 7.28(\mathrm{~d}, 1 \mathrm{H}, J=4 \mathrm{~Hz}, \mathrm{CH} 6), 7.29-7.32(\mathrm{~m}, 1 \mathrm{H}, \mathrm{CH} 8)$, $7.48(\mathrm{~s}, 1 \mathrm{H}, \mathrm{CH} 2), 7.54(\mathrm{dd}, 1 \mathrm{H}, J=4 \mathrm{~Hz}, J=8 \mathrm{~Hz}, \mathrm{CH} 9) .{ }^{13} \mathrm{C}-\mathrm{NMR} \delta\left(\mathrm{ppm}, \mathrm{DMSO}-d_{6}\right): 19.02\left(\mathrm{CH}_{3}\right.$ $\left.\gamma^{\prime}\right), 19.96\left(\mathrm{CH}_{3} \gamma\right), 31.19(\mathrm{CH} \beta), 56.06\left(\mathrm{OCH}_{3}\right.$ Phenyl), $56.16\left(\mathrm{OCH}_{3}\right.$ Phenyl), $59.54(\mathrm{CH} \alpha), 112.31(\mathrm{CH}$ Phenyl), 114.26 (CH Phenyl), 115.87 (CH 6), 122.35 (CH 7), 122.75 (Cq 1), 123.29 (CH Phenyl), 125.89 (Cq 5a), 126.49 (CH 8), 127.18 (CH 9), 130.95 (Cq Phenyl), 132.44 (CH 2), 133.40 (Cq 3a), 138.10 (Cq 9a), 147.60 (Cq 4), 149.25 (Cq Phenyl), 150.12 (Cq Phenyl), 170.00 (C=O). MS (ESI +, QTof, m/z): 420.9 [M + $\mathrm{H}]^{+}$. HRMS calculated for $\mathrm{C}_{23} \mathrm{H}_{25} \mathrm{~N}_{4} \mathrm{O}_{4} 421.1876$, found 421.1873 .

2-((1-(3,4-Dimethoxyphenyl)imidazo[1,2-a]quinoxalin-4-yl)amino)-4-methylpentanoic acid (10d): Following the same procedure used for the synthesis of $\mathbf{1 0 b}$, to a cooled solution of $\mathbf{8 d}(0.210 \mathrm{~g}, 0.43 \mathrm{mmol})$ in anhydrous $\mathrm{CH}_{2} \mathrm{Cl}_{2}(20 \mathrm{~mL})$ was added boron tribromide $(2.5 \mathrm{~mL}, 2.53 .6 \mathrm{mmol})$. The product was purified by flash chromatography eluted with cyclohexane/ethyl acetate 90/20 to 40/50. The compound is obtained as a white solid (76\% yield). $\mathrm{C}_{24} \mathrm{H}_{26} \mathrm{~N}_{4} \mathrm{O}_{4}$. MW: $434.49 \mathrm{~g} / \mathrm{mol}$. ${ }^{1} \mathrm{H}-\mathrm{NMR} \delta$ (ppm, DMSO- $\left.d_{6}\right): 0.95\left(\mathrm{~d}, 3 \mathrm{H}, J=8 \mathrm{~Hz}, \mathrm{CH}_{3} \delta\right), 0.98\left(\mathrm{~d}, 3 \mathrm{H}, J=8 \mathrm{~Hz}, \mathrm{CH}_{3} \delta^{\prime}\right), 1.76-1.80\left(\mathrm{~m}, 3 \mathrm{H}, \mathrm{CH}_{2} \beta\right.$, $\mathrm{CH} \gamma), 3.74$ (s, 3H, OCH 3 Phenyl), 3.87 (s, 3H, OCH 3 Phenyl), $4.66(\mathrm{t}, 1 \mathrm{H}, \mathrm{J}=4 \mathrm{~Hz}, \mathrm{CH} \alpha), 6.98-7.02$ (m, 1H, CH 7), 7.11 (d, 1H, J = 4 Hz, CH Phenyl), 7.13 (s, 1H, CH Phenyl), 7.17 (d, 1H, J = 4 Hz, CH Phenyl), $7.29(\mathrm{dd}, 1 \mathrm{H}, J=4 \mathrm{~Hz}, J=8 \mathrm{~Hz}, \mathrm{CH}$ 6), 7.31-7.33 (m, 1H, CH 8), 7.44 (d, 1H, J = $4 \mathrm{~Hz}, \mathrm{NH}), 7.49$ (s, $1 \mathrm{H}, \mathrm{CH} 2), 7.56(\mathrm{dd}, 1 \mathrm{H}, J=4 \mathrm{~Hz}, J=8 \mathrm{~Hz}, \mathrm{CH} 9) .{ }^{13} \mathrm{C}-\mathrm{NMR} \delta\left(\mathrm{ppm}, \mathrm{DMSO}-d_{6}\right): 22.68\left(\mathrm{CH}_{3} \delta\right), 23.69$ $\left(\mathrm{CH}_{3} \delta^{\prime}\right), 25.24(\mathrm{CH} \gamma), 40.42\left(\mathrm{CH}_{2} \beta\right), 53.00(\mathrm{CH} \alpha), 56.05\left(\mathrm{OCH}_{3}\right.$ Phenyl), $56.15\left(\mathrm{OCH}_{3}\right.$ Phenyl), 112.30 (CH Phenyl), 114.24 (CH Phenyl), 115.88 (CH 6), 122.29 (CH 7), 122.77 (Cq 1), 123.27 (CH Phenyl), 125.86 (Cq 5a), 126.49 (CH 8), 127.13 (CH 9), 130.90 (Cq 3a), 132.42 (CH 2), 138.12 (Cq 9a), 147.44 (Cq 4), 149.25 (Cq Phenyl), 150.10 (Cq Phenyl), 172.31 (C=O). MS (ESI +, QTof, m/z): 434.9 [M + H] ${ }^{+}$. HRMS calculated for $\mathrm{C}_{24} \mathrm{H}_{27} \mathrm{~N}_{4} \mathrm{O}_{4} 435.2032$, found 435.2032 .

2-((1-(3,4-Dihydroxyphenyl)imidazo[1,2-a]quinoxalin-4-yl)amino)acetic acid (11a): To a cooled $\left(0{ }^{\circ} \mathrm{C}\right)$ solution of $9 \mathrm{a}(0.055,0.14 \mathrm{mmol})$ in anhydrous $\mathrm{CH}_{2} \mathrm{Cl}_{2}(10 \mathrm{~mL})$ was added TFA $(5 \mathrm{~mL})$. The resulting solution was allowed to warm to room temperature and stirred until complete consumption of the starting material (1-2 h, monitored by TLC). The solvent was removed under reduced pressure. The product was purified by flash chromatography eluted with cyclohexane/ethyl acetate 90/20 to 40/50. The compound is obtained as a white solid (92\% yield). $\mathrm{C}_{18} \mathrm{H}_{14} \mathrm{~N}_{4} \mathrm{O}_{4}$. MW: $350.33 \mathrm{~g} / \mathrm{mol}$. ${ }^{1} \mathrm{H}-\mathrm{NMR} \delta$ (ppm, DMSO-d 6 ): $1.23(\mathrm{~s}, 1 \mathrm{H}, \mathrm{COOH}), 4.24\left(\mathrm{~d}, 2 \mathrm{H}, J=8 \mathrm{~Hz}, \mathrm{CH}_{2} \alpha\right), 6.82(\mathrm{dd}, 1 \mathrm{H}, J=4 \mathrm{~Hz}, J=8 \mathrm{~Hz}$, 
CH Phenyl), 6.90 (dd, 1H, $J=4 \mathrm{~Hz}, J=8 \mathrm{~Hz}, \mathrm{CH}$ Phenyl), 6.93 (s, 1H, CH Phenyl), 7.03-7.07 (m, 1H, $\mathrm{CH}$ 7), 7.31-7.34 (m, 1H, CH 8), $7.35(\mathrm{dd}, 1 \mathrm{H}, J=4 \mathrm{~Hz}, J=8 \mathrm{~Hz}, \mathrm{CH}$ 6), 7.48 (s, 1H, CH 2), 7.58 (dd, $1 \mathrm{H}$, $J=4 \mathrm{~Hz}, J=8 \mathrm{~Hz}, \mathrm{CH}$ 9), 8.02-8.05 (m, 1H, NH), 9.39-9.43 (m, 2H, C-OH Phenyl). ${ }^{13} \mathrm{C}-\mathrm{NMR} \delta$ (ppm, DMSO- $d_{6}$ ): $42.37\left(\mathrm{CH}_{2} \alpha\right), 115.97$ (CH 6), 116.49 (CH Phenyl), 117.86 (CH Phenyl), 120.69 (Cq 1), 122.06 (CH Phenyl), 122.96 (CH 7), 126.13 (Cq 5a), 126.51 (CH 8), 126.62 (CH 9), 132.36 (CH 2), 146.01 (Cq 3a), 147.17 (Cq 9a), 147.56 (Cq 4), 152.74 (Cq Phenyl), 172.09 (C=O). MS (ESI +, QTof, $m / z): 351.2[\mathrm{M}+\mathrm{H}]^{+}$. HRMS calculated for $\mathrm{C}_{18} \mathrm{H}_{15} \mathrm{~N}_{4} \mathrm{O}_{4} 351.1093$, found 351.1093.

2-((1-(3,4-dihydroxyphenyl)imidazo[1,2-a]quinoxalin-4-yl)amino)propanoic acid (11b): Following the same procedure for the synthesis of $11 \mathbf{a}$, to a cooled $\left(0^{\circ} \mathrm{C}\right)$ solution of $9 \mathbf{b}(0.040,0.09 \mathrm{mmol})$ in anhydrous $\mathrm{CH}_{2} \mathrm{Cl}_{2}(10 \mathrm{~mL})$ was added TFA $(5 \mathrm{~mL})$. The product was purified by flash chromatography eluted with cyclohexane/ethyl acetate $90 / 20$ to $40 / 50$. The compound is obtained as a white solid ( $88 \%$ yield). $\mathrm{C}_{19} \mathrm{H}_{16} \mathrm{~N}_{4} \mathrm{O}_{4}$. MW: $364.35 \mathrm{~g} / \mathrm{mol}^{1}{ }^{1} \mathrm{H}-\mathrm{NMR} \delta$ (ppm, DMSO- $\left.d_{6}\right): 1.23(\mathrm{~s}, 1 \mathrm{H}, \mathrm{COOH}), 1.56(\mathrm{~d}, 3 \mathrm{H}, J=8$ $\left.\mathrm{Hz}, \mathrm{CH}_{3} \beta\right), 4.82-4.84(\mathrm{~m}, 1 \mathrm{H}, \mathrm{CH} \alpha), 6.82(\mathrm{~d}, 1 \mathrm{H}, J=4 \mathrm{~Hz}, \mathrm{CH}$ Phenyl), $6.90(\mathrm{~d}, 1 \mathrm{H}, J=4 \mathrm{~Hz}, \mathrm{CH}$ Phenyl), 6.93 (s, 1H, CH Phenyl), 7.05-7.08 (m, 1H, CH 7), 7.33 (dd, 1H, J = 4 Hz, J = 8 Hz, CH 6), 7.35-7.37 (m, 1H, CH 8), 7.50 (s, 1H, CH 2), 7.59 (dd, 1H, J = 4 Hz, J = $8 \mathrm{~Hz}, \mathrm{CH}$ 9), $7.95(\mathrm{~d}, 1 \mathrm{H}, J=4 \mathrm{~Hz}$, $\mathrm{NH}), 9.37$ (s, 2H, C-OH Phenyl). ${ }^{13} \mathrm{C}-\mathrm{NMR} \delta$ (ppm, DMSO- $\left.d_{6}\right): 17.79\left(\mathrm{CH}_{3} \beta\right), 49.27(\mathrm{CH} \alpha), 116.01$ (CH 6), 116.51 (CH Phenyl), 117.87 (CH Phenyl), 120.42 (Cq 1), 120.95 (Cq 5a), 122.06 (CH Phenyl), 123.17 (CH 7), 125.80 (CH 9), 126.69 (CH 8), 131.82 (Cq 3a), 132.42 (CH 2), 132.64 (Cq 9a), 137.42 (Cq 4), 146.06 (Cq Phenyl), 146.95 (Cq Phenyl), 147.22 (Cq Phenyl), 174.64 (C=O). MS (ESI +, QTof, $m / z): 365.1$ $[\mathrm{M}+\mathrm{H}]^{+}$. HRMS calculated for $\mathrm{C}_{19} \mathrm{H}_{17} \mathrm{~N}_{4} \mathrm{O}_{4} 365.1250$, found 365.1240 .

2-((1-(3,4-Dihydroxyphenyl)imidazo[1,2-a]quinoxalin-4-yl)amino)-3-methylbutanoic acid (11c): Following the same procedure for the synthesis of $\mathbf{1 1 a}$, to a cooled $\left(0^{\circ} \mathrm{C}\right)$ solution of $9 \mathrm{c}(0.035,0.08 \mathrm{mmol})$ in anhydrous $\mathrm{CH}_{2} \mathrm{Cl}_{2}(10 \mathrm{~mL})$ was added TFA $(5 \mathrm{~mL})$. The product was purified by flash chromatography eluted with cyclohexane/ethyl acetate $90 / 20$ to $40 / 50$. The compound is obtained as a white solid (91\% yield). $\mathrm{C}_{21} \mathrm{H}_{20} \mathrm{~N}_{4} \mathrm{O}_{4}$. MW: $392.41 \mathrm{~g} / \mathrm{mol}^{1}{ }^{1} \mathrm{H}-\mathrm{NMR} \delta$ (ppm, DMSO- $d_{6}$ ): $1.04\left(\mathrm{~d}, 3 \mathrm{H}, J=8 \mathrm{~Hz}, \mathrm{CH}_{3}\right.$ $\gamma), 1.07\left(\mathrm{~d}, 3 \mathrm{H}, J=8 \mathrm{~Hz}, \mathrm{CH}_{3} \gamma^{\prime}\right), 1.18(\mathrm{~s}, 1 \mathrm{H}, \mathrm{COOH}), 2.36-2.41(\mathrm{~m}, 1 \mathrm{H}, \mathrm{CH} \beta), 4.74-4.76(\mathrm{~m}, 1 \mathrm{H}, \mathrm{CH}$

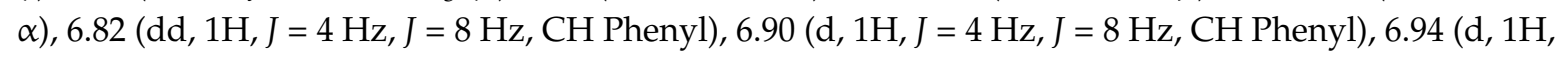
$J=4 \mathrm{~Hz}, J=8 \mathrm{~Hz}, \mathrm{CH}$ Phenyl), 7.06-7.10 (m, 1H, CH 7), $7.17(\mathrm{~d}, 1 \mathrm{H}, J=4 \mathrm{~Hz}, \mathrm{NH}), 7.33-7.36(\mathrm{~m}, 1 \mathrm{H}$, $\mathrm{CH} 8), 7.38(\mathrm{dd}, 1 \mathrm{H}, J=4 \mathrm{~Hz}, J=8 \mathrm{~Hz}, \mathrm{CH} 6), 7.51(\mathrm{~s}, 1 \mathrm{H}, \mathrm{CH} 2), 7.60(\mathrm{dd}, 1 \mathrm{H}, J=4 \mathrm{~Hz}, J=8 \mathrm{~Hz}, \mathrm{CH}$ 9), 9.40 (s, $2 \mathrm{H}, \mathrm{C}-\mathrm{OH}$ Phenyl). ${ }^{13} \mathrm{C}-\mathrm{NMR} \delta$ (ppm, DMSO- $\left.d_{6}\right): 18.86\left(\mathrm{CH}_{3} \gamma^{\prime}\right), 19.63\left(\mathrm{CH}_{3} \gamma\right), 30.50(\mathrm{CH}$ ß), $58.70(\mathrm{CH} \alpha), 115.98$ ( $\mathrm{CH}$ 6), 116.49 (CH Phenyl), 117.86 (CH Phenyl), 120.83 (Cq 1), 122.07 ( $\mathrm{CH}$ Phenyl), 123.29 (CH 7), 126.05 (Cq 5a), 126.72 (CH 8), 126.94 (CH 9), 132.12 (CH 2), 132.52 (Cq Phenyl), 132.82 (Cq 3a), 137.42 (Cq 9a), 146.06 (Cq 4), 147.23 (Cq Phenyl), 147.47 (Cq Phenyl), 171.62 (C=O). MS (ESI +, QTof, $m / z)$ : $393.2[\mathrm{M}+\mathrm{H}]^{+}$. HRMS calculated for $\mathrm{C}_{21} \mathrm{H}_{21} \mathrm{~N}_{4} \mathrm{O}_{4} 393.1563$, found 393.1561.

2-((1-(3,4-Dihydroxyphenyl)imidazo[1,2-a]quinoxalin-4-yl)amino)-4-methylpentanoic acid (11d): Following the same procedure for the synthesis of $\mathbf{1 1 a}$, to a cooled $\left(0{ }^{\circ} \mathrm{C}\right)$ solution of $\mathbf{9 d}(0.050,0.11 \mathrm{mmol})$ in anhydrous $\mathrm{CH}_{2} \mathrm{Cl}_{2}(10 \mathrm{~mL})$ was added TFA $(5 \mathrm{~mL})$. The product was purified by flash chromatography eluted with cyclohexane/ethyl acetate $90 / 20$ to $40 / 50$. The compound is obtained as a white solid (78\% yield). $\mathrm{C}_{22} \mathrm{H}_{22} \mathrm{~N}_{4} \mathrm{O}_{4}$. MW: $406.43 \mathrm{~g} / \mathrm{mol} .{ }^{1} \mathrm{H}-\mathrm{NMR} \delta$ (ppm, DMSO- $\left.d_{6}\right): 0.93\left(\mathrm{~d}, 3 \mathrm{H}, J=8 \mathrm{~Hz}, \mathrm{CH}_{3} \delta\right.$ ), $0.98\left(\mathrm{~d}, 3 \mathrm{H}, J=8 \mathrm{~Hz}, \mathrm{CH}_{3} \delta^{\prime}\right), 1.42(\mathrm{~s}, 1 \mathrm{H}, \mathrm{COOH}), 1.69-1.72\left(\mathrm{~m}, 1 \mathrm{H}, \mathrm{CH}_{2} \beta\right), 1.76-1.79(\mathrm{~m}, 1 \mathrm{H}, \mathrm{CH} \gamma)$, 1.99-2.02 (m, 1H, CH $2 \beta), 4.85(\mathrm{t}, 1 \mathrm{H}, J=4 \mathrm{~Hz}, \mathrm{CH} \alpha), 6.82(\mathrm{~d}, 1 \mathrm{H}, J=4 \mathrm{~Hz}, \mathrm{CH}$ Phenyl), $6.90(\mathrm{~s}, 1 \mathrm{H}, \mathrm{CH}$ Phenyl), 6.93 (d, 1H, J = 4 Hz, CH Phenyl), 7.05-7.08 (m, 1H, CH 7), 7.32-7.35 (m, 1H, CH 8), 7.36 (dd, $1 \mathrm{H}, J=4 \mathrm{~Hz}, J=8 \mathrm{~Hz}, \mathrm{CH}$ ) $), 7.50(\mathrm{~s}, 1 \mathrm{H}, \mathrm{CH}$ ) $), 7.59(\mathrm{dd}, 1 \mathrm{H}, J=4 \mathrm{~Hz}, J=8 \mathrm{~Hz}, \mathrm{CH} 9), 7.90(\mathrm{~d}, 1 \mathrm{H}, J=4$ $\mathrm{Hz}, \mathrm{NH}), 9.38$ (s, 2H, C-OH Phenyl). ${ }^{13} \mathrm{C}-\mathrm{NMR} \delta$ (ppm, DMSO- $\left.d_{6}\right): 20.74\left(\mathrm{CH}_{3} \delta\right), 22.28\left(\mathrm{CH}_{3} \delta^{\prime}\right), 24.07$ $(\mathrm{CH} \gamma), 39.08\left(\mathrm{CH}_{2} \beta\right), 50.92(\mathrm{CH} \alpha), 114.92(\mathrm{CH} 6), 115.42$ (CH Phenyl), 116.77 (CH Phenyl), 119.82 (Cq 1), 120.98 (CH Phenyl), 122.10 (CH 7), 124.67 (Cq 5a), 125.64 (CH 8), 125.72 (CH 9), 130.79 (Cq 3a), 131.28 (CH 2), 144.99 (Cq 9a), 146.14 (Cq 4), 146.28 (Cq Phenyl), 157.42 (Cq Phenyl), 173.51 (C=O). MS $($ ESI +, QTof, $m / z)$ : $407.1[\mathrm{M}+\mathrm{H}]^{+}$. HRMS calculated for $\mathrm{C}_{22} \mathrm{H}_{23} \mathrm{~N}_{4} \mathrm{O}_{4} 407.1719$, found 407.1712. 
6-Amino-2-((1-(3,4-dihydroxyphenyl)imidazo[1,2-a]quinoxalin-4-yl)amino)hexanoic acid (11e): Following the same procedure for the synthesis of 11a. To a cooled $\left(0^{\circ} \mathrm{C}\right)$ solution of $9 \mathbf{e}(0.035,0.06 \mathrm{mmol})$ in anhydrous $\mathrm{CH}_{2} \mathrm{Cl}_{2}(10 \mathrm{~mL})$ was added TFA $(5 \mathrm{~mL})$. The product was purified by flash chromatography eluted with cyclohexane/ethyl acetate $90 / 20$ to $40 / 50$. The compound is obtained as a white solid ( $84 \%$ yield). $\mathrm{C}_{22} \mathrm{H}_{23} \mathrm{~N}_{5} \mathrm{O}_{4}$. MW: $421.45 \mathrm{~g} / \mathrm{mol} .{ }^{1} \mathrm{H}-\mathrm{NMR} \delta$ (ppm, DMSO- $d_{6}$ ): 1.23 (s, $1 \mathrm{H}, \mathrm{COOH}$ ), $1.47-1.51$ $\left(\mathrm{m}, 2 \mathrm{H}, \mathrm{CH}_{2} \gamma\right), 1.58-1.64\left(\mathrm{~m}, 2 \mathrm{H}, \mathrm{CH}_{2} \delta\right), 1.98-2.04\left(\mathrm{~m}, 2 \mathrm{H}, \mathrm{CH}_{2} \beta\right), 2.79-2.83\left(\mathrm{~m}, 2 \mathrm{H}, \mathrm{CH}_{2} \varepsilon\right), 4.76-4.78$ $(\mathrm{m}, 1 \mathrm{H}, \mathrm{CH} \alpha), 6.81(\mathrm{~d}, 1 \mathrm{H}, J=4 \mathrm{~Hz}, \mathrm{CH}$ Phenyl), 6.90 (s, 1H, CH Phenyl), 6.93 (d, 1H, J = 4 Hz, CH Phenyl), 7.03-7.07 (m, 1H, CH 7), 7.31-7.35 (m, 1H, CH 8), 7.37 (dd, 1H, J = 4 Hz, J = 8 Hz, CH 6), 7.47 $(\mathrm{s}, 1 \mathrm{H}, \mathrm{CH} 2), 7.58(\mathrm{dd}, 1 \mathrm{H}, J=4 \mathrm{~Hz}, J=8 \mathrm{~Hz}, \mathrm{CH} 9), 7.64(\mathrm{~d}, 1 \mathrm{H}, J=4 \mathrm{~Hz}, \mathrm{NH}-\mathrm{CH} \alpha), 7.68-7.70(\mathrm{~m}, 2 \mathrm{H}$, $\left.\mathrm{NH}_{2}\right), 9.40-9.46$ (m, 2H, C-OH Phenyl). ${ }^{13} \mathrm{C}-\mathrm{NMR} \delta$ (ppm, DMSO- $\left.d_{6}\right): 22.04\left(\mathrm{CH}_{2} \gamma\right), 26.08\left(\mathrm{CH}_{2} \delta\right)$, $29.65\left(\mathrm{CH}_{2} \beta\right), 38.04\left(\mathrm{CH}_{2} \varepsilon\right), 52.17(\mathrm{CH} \alpha), 114.84(\mathrm{CH}$ ) $), 115.40$ (CH Phenyl), 116.79 (CH Phenyl), 120.96 (CH Phenyl), 121.87 (CH 7), 124.88 (Cq 5a), 125.49 (CH 8), 126.01 (CH 9), 130.49 (Cq 1), 131.14 (CH 2), 131.69 (Cq 3a), 136.24 (Cq 9a), 145.00 (Cq 4), 146.10 (Cq Phenyl), 146.51 (Cq Phenyl), 157.59 (Cq Phenyl), $173.29(\mathrm{C}=\mathrm{O})$. MS (ESI +, QTof, $m / z): 422.1[\mathrm{M}+\mathrm{H}]^{+}$. HRMS calculated for $\mathrm{C}_{22} \mathrm{H}_{24} \mathrm{~N}_{5} \mathrm{O}_{4}$ 422.1828, found 422.1834 .

5-Amino-2-((1-(3,4-dihydroxyphenyl)imidazo[1,2-a]quinoxalin-4-yl)amino)pentanoic acid (11f): Following the same procedure for the synthesis of $\mathbf{1 1 a}$, to a cooled $\left(0{ }^{\circ} \mathrm{C}\right)$ solution of $9 \mathrm{f}(0.055,0.09 \mathrm{mmol})$ in anhydrous $\mathrm{CH}_{2} \mathrm{Cl}_{2}(10 \mathrm{~mL})$ was added TFA $(5 \mathrm{~mL})$. The product was purified by flash chromatography eluted with cyclohexane/ethyl acetate $90 / 20$ to $40 / 50$. The compound is obtained as a white solid (10\% yield). $\mathrm{C}_{21} \mathrm{H}_{21} \mathrm{~N}_{5} \mathrm{O}_{4}$. MW: $407.42 \mathrm{~g} / \mathrm{mol} .{ }^{1} \mathrm{H}-\mathrm{NMR} \delta$ (ppm, DMSO- $d_{6}$ ): ${ }^{1} \mathrm{H}-\mathrm{NMR} \delta$ (ppm, $400 \mathrm{MHz}$, DMSO- $\left.d_{6}\right): 1.70-1.74\left(\mathrm{~m}, 2 \mathrm{H}, \mathrm{CH}_{2} \gamma\right), 1.89-1.93\left(\mathrm{~m}, 1 \mathrm{H}, \mathrm{CH}_{2} \beta\right), 2.07-2.11\left(\mathrm{~m}, 1 \mathrm{H}, \mathrm{CH}_{2} \beta\right), 2.66-2.70(\mathrm{~m}$, $\left.1 \mathrm{H}, \mathrm{CH}_{2} \delta\right), 2.78-2.82\left(\mathrm{~m}, 1 \mathrm{H}, \mathrm{CH}_{2} \delta\right), 4.33-4.34(\mathrm{~m}, 1 \mathrm{H}, \mathrm{CH} \alpha), 6.69(\mathrm{~d}, 1 \mathrm{H}, J=8 \mathrm{~Hz}, \mathrm{CH}$ Phenyl), 6.80 $(\mathrm{d}, 1 \mathrm{H}, J=8 \mathrm{~Hz}, \mathrm{CH}$ Phenyl), $6.89(\mathrm{~d}, 1 \mathrm{H}, J=4 \mathrm{~Hz}, \mathrm{CH}$ Phenyl), 6.97-7.01 (m, 1H, CH 7), 7.29-.33 (m, 1H, $\mathrm{CH} 8), 7.36(\mathrm{dd}, 1 \mathrm{H}, J=4 \mathrm{~Hz}, J=8 \mathrm{~Hz}, \mathrm{CH} 6), 7.40(\mathrm{~s}, 1 \mathrm{H}, J=4 \mathrm{~Hz}, \mathrm{CH} 2), 7.46(\mathrm{~d}, 1 \mathrm{H}, J=4 \mathrm{~Hz}, \mathrm{NH}-\mathrm{CH}$ $\alpha), 7.57(\mathrm{dd}, 1 \mathrm{H}, J=4 \mathrm{~Hz}, J=8 \mathrm{~Hz}, \mathrm{CH} 9), 8.69$ (s, 2H, C-OH Phenyl). ${ }^{13} \mathrm{C}-\mathrm{NMR} \delta$ (ppm, DMSO- $d_{6}$ ): $23.61\left(\mathrm{CH}_{2} \gamma\right), 29.41\left(\mathrm{CH}_{2} \beta\right), 40.41\left(\mathrm{CH}_{2} \delta\right), 54.57(\mathrm{CH} \alpha), 115.88(\mathrm{CH} 6), 116.50$ (CH Phenyl), 117.95 (CH Phenyl), 121.23 (Cq 1), 122.04 (CH 7), 122.18 (CH Phenyl), 125.85 (Cq 5a), 126.39 (CH 8), 127.17 (CH 9), 132.08 (CH 2), 133.25 (Cq 3a), 138.31 (Cq 9a), 146.07 (Cq 4), 146.84 (Cq Phenyl), 147.12 (Cq Phenyl), $174.19(\mathrm{C}=\mathrm{O})$. MS (ESI +, QTof, $m / z)$ : $408.2[\mathrm{M}+\mathrm{H}]^{+}$. HRMS calculated for $\mathrm{C}_{21} \mathrm{H}_{22} \mathrm{~N}_{5} \mathrm{O}_{4}$ 408.1672, found 408.1668 .

2-((1-(3,4-Dihydroxyphenyl)imidazo[1,2-a]quinoxalin-4-yl)amino)-3-phenylpropanoic acid (11g): Following the same procedure for the synthesis of $\mathbf{1 1 a}$, to a cooled $\left(0{ }^{\circ} \mathrm{C}\right)$ solution of $9 \mathrm{~g}(0.045,0.09 \mathrm{mmol})$ in anhydrous $\mathrm{CH}_{2} \mathrm{Cl}_{2}(10 \mathrm{~mL})$ was added TFA $(5 \mathrm{~mL})$. The product was purified by flash chromatography eluted with cyclohexane/ethyl acetate $90 / 20$ to $40 / 50$. The compound is obtained as a white solid ( $87 \%$ yield). $\mathrm{C}_{25} \mathrm{H}_{20} \mathrm{~N}_{4} \mathrm{O}_{4}$. MW: $440.45 \mathrm{~g} / \mathrm{mol} .{ }^{1} \mathrm{H}-\mathrm{NMR} \delta$ (ppm, DMSO- $d_{6}$ ): 1.23 (s, $1 \mathrm{H}, \mathrm{COOH}$ ), 3.33-3.39 $\left(\mathrm{m}, 2 \mathrm{H}, \mathrm{CH}_{2} \beta\right), 5.04-5.07(\mathrm{~m}, 1 \mathrm{H}, \mathrm{CH} \alpha), 6.81(\mathrm{~d}, 1 \mathrm{H}, J=8 \mathrm{~Hz}, \mathrm{CH}$ Phenyl), $6.89(\mathrm{~d}, 1 \mathrm{H}, J=4 \mathrm{~Hz}, \mathrm{CH}$ Phenyl), 6.91 (d, 1H, J = 8 Hz, CH Phenyl), 7.05-7.08 (m, 1H, CH 7), 7.17 (t, 1H, J = 8 Hz, CH Phenyl), $7.26(\mathrm{t}, 2 \mathrm{H}, J=8 \mathrm{~Hz}, \mathrm{CH}$ Phenyl), 7.31-7.333 (m, 1H, CH 8), $7.34(\mathrm{dd}, 1 \mathrm{H}, J=4 \mathrm{~Hz}, J=8 \mathrm{~Hz}, \mathrm{CH} 6)$, 7.36-7.37 (m, 2H, CH Phenyl), $7.48(\mathrm{~s}, 1 \mathrm{H}, \mathrm{CH} 2), 7.61(\mathrm{dd}, 1 \mathrm{H}, J=4 \mathrm{~Hz}, J=8 \mathrm{~Hz}, \mathrm{CH} 9), 7.76-7.77$ $(\mathrm{m}, 1 \mathrm{H}, \mathrm{NH}), 9.39$ (s, 2H, C-OH Phenyl). ${ }^{13} \mathrm{C}-\mathrm{NMR} \delta$ (ppm, DMSO-d $\left.)_{6}\right): 35.61\left(\mathrm{CH}_{2} \beta\right), 5.31(\mathrm{CH} \alpha)$, 114.91 (CH 6), 115.40 (CH Phenyl), 116.75 (CH Phenyl), 119.75 (Cq 1), 120.96 (CH Phenyl), 122.23 (CH 7), 124.71 (Cq 5a), 125.64 (CH 9), 125.88 (CH 8), 127.67 (CH Phenyl), 128.58 (CH Phenyl), 130.78 (Cq Phenyl), 131.35 (CH 2), 137.26 (Cq 3a), 138.42 (Cq 9a), 144.98 (Cq Phenyl), 146.14 (Cq 4), 157.48 (Cq Phenyl), 157.77 (Cq Phenyl), 172.35 (C=O). MS (ESI +, QTof, $m / z)$ : $441.2[\mathrm{M}+\mathrm{H}]^{+}$. HRMS calculated for $\mathrm{C}_{25} \mathrm{H}_{21} \mathrm{~N}_{4} \mathrm{O}_{4} 441.1563$, found 441.1569 .

2-((1-(3,4-Dihydroxyphenyl)imidazo[1,2-a]quinoxalin-4-yl)amino)-3-(4-hydroxyphenyl)-propanoic acid (11h): Following the same procedure for the synthesis of $\mathbf{1 1 a}$, to a cooled $\left(0{ }^{\circ} \mathrm{C}\right)$ solution of $\mathbf{9 h}(0.030$, $0.06 \mathrm{mmol})$ in anhydrous $\mathrm{CH}_{2} \mathrm{Cl}_{2}(10 \mathrm{~mL})$ was added TFA $(5 \mathrm{~mL})$. The product was purified by flash chromatography eluted with cyclohexane/ethyl acetate $90 / 20$ to $40 / 50$. The compound is obtained as 
a white solid (89\% yield). $\mathrm{C}_{25} \mathrm{H}_{20} \mathrm{~N}_{4} \mathrm{O}_{5} . \mathrm{MW}: 456.45 \mathrm{~g} / \mathrm{mol} .{ }^{1} \mathrm{H}-\mathrm{NMR} \delta$ (ppm, DMSO- $\left.d_{6}\right): 1.29(\mathrm{~s}, 1 \mathrm{H}$, $\mathrm{COOH}), 3.21-3.23\left(\mathrm{~m}, 2 \mathrm{H}, \mathrm{CH}_{2} \beta\right), 4.94-4.98(\mathrm{~m}, 1 \mathrm{H}, \mathrm{CH} \alpha), 6.71(\mathrm{~d}, 2 \mathrm{H}, J=8 \mathrm{~Hz}, \mathrm{CH}$ Phenyl), 6.86 $(\mathrm{dd}, 1 \mathrm{H}, J=4 \mathrm{~Hz}, J=8 \mathrm{~Hz}, \mathrm{CH}$ Phenyl), $6.94(\mathrm{~d}, 1 \mathrm{H}, \mathrm{CH}$ Phenyl), 6.98 (d, 1H, $J=8 \mathrm{~Hz}, \mathrm{CH}$ Phenyl), 7.04-7.08 (m, 1H, CH 7), 7.12 (d, 2H, $J=8$ Hz, CH Phenyl), 7.31-7.32 (m, 1H, CH 8), 7.35 (dd, 1H, $J=4$ $\mathrm{Hz}, J=8 \mathrm{~Hz}, \mathrm{CH} 6), 7.47(\mathrm{~s}, 1 \mathrm{H}, \mathrm{CH} 2), 7.59-7.60(\mathrm{~m}, 1 \mathrm{H}, \mathrm{NH}), 7.64(\mathrm{dd}, 1 \mathrm{H}, J=4 \mathrm{~Hz}, J=8 \mathrm{~Hz}, \mathrm{CH} 9)$, 9.32-9.35 (m, 2H, C-OH Phenyl). ${ }^{13} \mathrm{C}-\mathrm{NMR} \delta$ (ppm, DMSO- $\left.d_{6}\right): 36.01\left(\mathrm{CH}_{2} \beta\right), 55.24(\mathrm{CH} \alpha), 115.56$ $(2 \times \mathrm{CH}$ Phenyl), 115.96 (CH 6), 116.47 (CH Phenyl), 117.82 (CH Phenyl), 120.86 (Cq 1), 122.05 (CH Phenyl), 123.19 (CH 7), 125.81 (Cq 5a), 126.68 (CH 9), 126.70 (CH 8), 130.59 (2 × CH Phenyl), 132.35 (Cq 3a), 132.48 (CH 2), 137.32 (Cq 9a), 146.03 (Cq 4), 147.05 (Cq Phenyl), 147.19 (Cq Phenyl), 156.44 (Cq Phenyl), $173.55(\mathrm{C}=\mathrm{O})$. MS (ESI +, QTof, $m / z): 457.1[\mathrm{M}+\mathrm{H}]^{+}$. HRMS calculated for $\mathrm{C}_{25} \mathrm{H}_{21} \mathrm{~N}_{4} \mathrm{O}_{5}$ 457.1512, found 457.1514.

2-Amino-5-((1-(3,4-dihydroxyphenyl)imidazo[1,2-a]quinoxalin-4-yl)amino)pentanoic acid (11i): Following the same procedure for the synthesis of $11 \mathrm{a}$, to a cooled $\left(0^{\circ} \mathrm{C}\right)$ solution of $9 \mathbf{i}$ in anhydrous $\mathrm{CH}_{2} \mathrm{Cl}_{2}$ $(10 \mathrm{~mL})$ was added TFA $(5 \mathrm{~mL})$. The product was purified by flash chromatography eluted with cyclohexane/ethyl acetate $90 / 20$ to $40 / 50$. The compound is obtained as a white solid (79\% yield). $\mathrm{C}_{21} \mathrm{H}_{21} \mathrm{~N}_{5} \mathrm{O}_{4}$. MW: $407.42 \mathrm{~g} / \mathrm{mol}^{1}{ }^{1} \mathrm{H}-\mathrm{NMR} \delta$ (ppm, DMSO- $\left.d_{6}\right): 1.23(\mathrm{~s}, 1 \mathrm{H}, \mathrm{COOH}), 1.85-1.89(\mathrm{~m}, 4 \mathrm{H}$, $\left.\mathrm{CH}_{2} \gamma, \mathrm{CH}_{2} \beta\right), 3.64-3.66\left(\mathrm{~m}, 2 \mathrm{H}, \mathrm{CH}_{2} \delta\right), 3.99-4.01(\mathrm{~m}, 1 \mathrm{H}, \mathrm{CH} \alpha), 6.80(\mathrm{dd}, 1 \mathrm{H}, J=4 \mathrm{~Hz}, J=8 \mathrm{~Hz}, \mathrm{CH}$ Phenyl), $6.89(\mathrm{~d}, 1 \mathrm{H}, J=4 \mathrm{~Hz}, \mathrm{CH}$ Phenyl), $6.92(\mathrm{~d}, 1 \mathrm{H}, J=8 \mathrm{~Hz}, \mathrm{CH}$ Phenyl), 7.07-7.11 (m, 1H, CH 7), $7.35(\mathrm{dd}, 1 \mathrm{H}, J=4 \mathrm{~Hz}, J=8 \mathrm{~Hz}, \mathrm{CH} 6), 7.37-7.39(\mathrm{~m}, 1 \mathrm{H}, \mathrm{CH}$ ) $), 7.51(\mathrm{~s}, 1 \mathrm{H}, \mathrm{CH} 2), 7.55$ (dd, $1 \mathrm{H}, J=4$ $\mathrm{Hz}, J=8 \mathrm{~Hz}, \mathrm{CH}$ 9), $7.66\left(\mathrm{t}, 1 \mathrm{H}, J=4 \mathrm{~Hz}, \mathrm{NH}-\mathrm{CH}_{2} \delta\right), 8.24\left(\mathrm{~s}, 2 \mathrm{H}, \mathrm{NH}_{2}\right), 9.40$ (s, 2H, C-OH Phenyl). ${ }^{13} \mathrm{C}-\mathrm{NMR} \delta$ (ppm, DMSO-d 6$): 24.80\left(\mathrm{CH}_{2} \gamma\right), 28.04\left(\mathrm{CH}_{2} \beta\right), 39.99\left(\mathrm{CH}_{2} \delta\right), 52.30(\mathrm{CH} \alpha), 116.12(\mathrm{CH} 6)$, 116.51 (CH Phenyl), 117.77 (CH Phenyl), 120.74 (Cq 1), 121.96 (CH Phenyl), 123.27 (CH 7), 125.76 (Cq 5a), 126.83 (CH 8), 129.28 (CH 9), 132.68 (CH 2), 138.42 (Cq 3a), 146.12 (Cq 4), 147.29 (Cq Phenyl), 158.41 (Cq Phenyl), 158.75 (Cq 9a), 171.54 (C=O). MS (ESI +, QTof, $m / z): 408.2[\mathrm{M}+\mathrm{H}]^{+}$. HRMS calculated for $\mathrm{C}_{21} \mathrm{H}_{22} \mathrm{~N}_{5} \mathrm{O}_{4}$ 408.1672, found 408.1668.

\subsubsection{3,4-Dihydroxyphenylboronic Acid (12)}

To a cooled $\left(0^{\circ} \mathrm{C}\right)$ solution of 3,4-dimethoxyphenylboronic acid $(0.800 \mathrm{~g}, 4.39 \mathrm{mmol})$ in anhydrous $\mathrm{CH}_{2} \mathrm{Cl}_{2}(50 \mathrm{~mL})$ was added boron tribromide $(10 \mathrm{~mL}, 10 \mathrm{mmol})$. The resulting solution was allowed to warm to room temperature and stirred until complete consumption of the starting material (1-2h, monitored by TLC). The solution was neutralized by addition of methanol $(50 \mathrm{~mL})$. The crude mixture was concentrated under reduced pressure. The compound was obtained as a white solid (84\% yield) and used without purification. $\mathrm{C}_{6} \mathrm{H}_{7} \mathrm{BO}_{4}$. MW: $153.93 \mathrm{~g} / \mathrm{mol} .{ }^{1} \mathrm{H}-\mathrm{NMR} \delta$ (ppm, DMSO- $\left.d_{6}\right): 2.08(\mathrm{~s}, 2 \mathrm{H}$, B-OH), 6.47 (d, 1H, CH Phenyl), 6.60 (d, 1H, CH Phenyl), 6.71 (d, 1H, CH Phenyl), 8.00 (s, 2H, C-OH Phenyl). ${ }^{13}$ C-NMR $\delta$ (ppm, DMSO- $d_{6}$ ): 108.10 (CH Phenyl), 116.14 (CH Phenyl), 119.73(CH Phenyl), $145.73(\mathrm{C}-\mathrm{OH})$. MS (ESI +, QTof, $\mathrm{m} / \mathrm{z}): 153.2[\mathrm{M}-\mathrm{H}]^{-}$. HRMS calculated for $\mathrm{C}_{6} \mathrm{H}_{6} \mathrm{O}_{4} \mathrm{~B}$ 153.0359, found 153.0358.

\subsection{Cell Line and Culture Techniques}

The melanoma (A375) human cancer cell line is obtained from American Type Culture Collection (Rockville, MD, USA). Cells were cultured in RPMI Gibco medium containing RPMI-1640 (Waltham, MA, USA), $10 \%$ heat-inactived $\left(56^{\circ} \mathrm{C}\right)$ foetal bovine serum (FBS) (Polylabo, Paris, France), $2 \mathrm{mM}$ L-glutamine, $100 \mathrm{IU} / \mathrm{mL}$ penicillin $\mathrm{G}$ sodium, $100 \mathrm{mg} / \mathrm{mL}$ streptomycin sulfate, and $0.25 \mathrm{mg} / \mathrm{mL}$ amphotericin B. Cells were maintained in a humidified atmosphere of $5 \% \mathrm{CO}_{2}$ in air at $37^{\circ} \mathrm{C}$.

\subsection{In Vitro Cytotoxicity Assay}

Previously to the experiments, the number of cells by well, the doubling time and the MTT concentration have been optimized. In all the experiments, A375 cells were seeded at a final concentration of 5000 cells/well in 96-well microtiter plates and allowed to attach overnight. After 
$24 \mathrm{~h}$ incubation, the medium (phosphate-buffer saline $\mathrm{pH}$ 7.3) was aspirated carefully from the plates using a sterile Pasteur pipette, and cells were exposed (i) to vehicle controls $(0.15 \%$ DMSO/culture medium $(v / v)$ and culture medium alone), (ii) to EAPB02303, EAPB02302 and the synthesized compounds at concentrations of $10^{-5}-3.2 \times 10^{-9} \mathrm{M}$ dissolved in a mixture $0.15 \% \mathrm{DMSO} /$ culture medium $(v / v)$. After $96 \mathrm{~h}$ of incubation, cell supernatant was removed and $100 \mu \mathrm{L}$ of a MTT (3-[4.5-dimethylthiazol-2-yl]-2.5-diphenyltetrazolium bromide) solution in fresh medium was added per well (MTT final concentration of $0.5 \mathrm{mg} / \mathrm{mL}$ ) and incubated for $4 \mathrm{~h}$ at $37^{\circ} \mathrm{C}$. This colorimetric assay is based on the ability of live and metabolically unimpaired tumor-cell targets to reduce MTT to a blue formazan product. At the end of the incubation period, the supernatant was carefully aspirated, then, $100 \mu \mathrm{L}$ of a mixture of isopropyl alcohol and $1 \mathrm{M}$ hydrochloric acid $(96 / 4, v / v)$ was added to each well. After $10 \mathrm{~min}$ of incubation and vigorous shaking to solubilize formazan crystals, the optical density was measured at $570 \mathrm{nM}$ in a microculture plate reader (Zaragoza, Spain). For each assay, at least three experiments were performed in triplicate. The individual cell line growth curves confirmed that all A375 line in control medium remained in the log phase of cell growth $96 \mathrm{~h}$ after plating. Cell survival was expressed as percent of vehicle control. The $\mathrm{IC}_{50}$ values defined as the concentrations of drugs which produced $50 \%$ cell growth inhibition; $50 \%$ reduction of absorbance, were estimated from the sigmoidal dose-response curves.

\section{Conclusions}

The synthesis and study of the amino acid groups grafted on position 4 within the imiqualine series highlight the fact that the nature of the substituent on position 4 is not essential for the biological activity. Indeed, large modifications between ЕAPB02302, which only has a primary amine, and the new compounds with a complete amino acid residue do not significantly modify the activity, which remains similar to that of our first imiqualine generation. However, these modulations allow one to significantly increase the theoretical water solubility. The presence of dihydroxy groups on the phenyl appears to be necessary for the conservation of the cytotoxic activity on the melanoma cell line tested. These encouraging results obtained on the representative A375 melanoma cell line will prompt us to study further in vivo evaluation on xenografted mice.

Supplementary Materials: The following are available online. NMR ${ }^{1} \mathrm{H}$ and ${ }^{13} \mathrm{C}$ spectra of all compounds evaluated on A375 melanoma cells.

Author Contributions: Conceptualization, A.C., C.P., P.-A.B. and C.D.-M.; Investigation, A.C.; Methodology, A.C., C.P. and C.D.-M.; Supervision, P.C., P.-A.B. and C.D.-M.; Writing-original draft, A.C. and C.P.; Writing-review \& editing, P.-A.B. and C.D.-M.

Funding: This research was funded by the Société d'Accélération du Transfert de Technologies (SATT AxLr).

Acknowledgments: Authors thank the Société d'Accélération du Transfert de Technologies (SATT AxLr) for financial support to Cindy Patinote, Stephanie Paniagua and Amandine Dejean for their technical help, AGV Discovery for the biological study, and the French department of Biotage for lending us a HPFC system.

Conflicts of Interest: The authors declare no conflict of interest.

\section{References}

1. Eggermont, A.M.M.; Spatz, A.; Robert, C. Cutaneous melanoma. Lancet Lond. Engl. 2014, 383, $816-827$. [CrossRef]

2. Stratigos, A.; Garbe, C.; Lebbe, C.; Malvehy, J.; del Marmol, V.; Pehamberger, H.; Peris, K.; Becker, J.C.; Zalaudek, I.; Saiag, P.; et al. European Dermatology Forum (EDF); European Association of Dermato-Oncology (EADO); European Organization for Research and Treatment of Cancer (EORTC) Diagnosis and treatment of invasive squamous cell carcinoma of the skin: European consensus-based interdisciplinary guideline. Eur. J. Cancer Oxf. Engl. 2015, 51, 1989-2007. [CrossRef]

3. Kwong, A.; Sanlorenzo, M.; Rappersberger, K.; Vujic, I. Update on advanced melanoma treatments: Small molecule targeted therapy, immunotherapy, and future combination therapies. Wien. Med. Wochenschr. 2017, 1-9. [CrossRef] [PubMed] 
4. Drugs Approved for Melanoma. Available online: https://www.cancer.gov/about-cancer/treatment/ drugs / melanoma (accessed on 11 October 2018).

5. Glitza, I.C.; Kim, D.W.; Chae, Y.K.; Kim, K.B. Targeted Therapy in Melanoma. In Genetics of Melanoma; Cancer Genetics; Springer: New York, NY, USA, 2016; pp. 237-265. ISBN 978-1-4939-3552-9.

6. Keller, H.R.; Zhang, X.; Li, L.; Schaider, H.; Wells, J.W. Overcoming resistance to targeted therapy with immunotherapy and combination therapy for metastatic melanoma. Oncotarget 2017, 8, 75675-75686. [CrossRef] [PubMed]

7. Basken, J.; Stuart, S.A.; Kavran, A.J.; Lee, T.; Ebmeier, C.C.; Old, W.; Ahn, N.G. Specificity of phosphorylation responses to MAP kinase pathway inhibitors in melanoma cells. Mol. Cell. Proteomics MCP 2017. [CrossRef]

8. Moarbess, G.; Deleuze-Masquefa, C.; Bonnard, V.; Gayraud-Paniagua, S.; Vidal, J.-R.; Bressolle, F.; Pinguet, F.; Bonnet, P.-A. In vitro and in vivo anti-tumoral activities of imidazo[1,2-a]quinoxaline, imidazo[1,5-a]quinoxaline, and pyrazolo[1,5-a]quinoxaline derivatives. Bioorg. Med. Chem. 2008, 16, 6601-6610. [CrossRef] [PubMed]

9. Deleuze-Masquefa, C.; Moarbess, G.; Khier, S.; David, N.; Gayraud-Paniagua, S.; Bressolle, F.; Pinguet, F.; Bonnet, P.-A. New imidazo[1,2-a]quinoxaline derivatives: Synthesis and in vitro activity against human melanoma. Eur. J. Med. Chem. 2009, 44, 3406-3411. [CrossRef] [PubMed]

10. Deleuze-Masquefa, C.; Moarbess, G.; Bonnet, P.-A.; Pinguet, F.; Bazarbachi, A.; Bressolle, F. Imidazo[1,2-a]quinoxalines and derivatives for the treatment of cancers. Patent WO2009043934 A1, 2009.

11. Cuq, P.; Deleuze-Masquefa, C.; Bonnet, P.-A.; Patinote, C. New Imidazo[1,2-a]quinoxalines and Derivatives for the Treatment of Cancers. Patent WO2016107895 A1, 2016.

12. Chouchou, A.; Marion, B.; Enjalbal, C.; Roques, C.; Cuq, P.; Bonnet, P.-A.; Bressolle-Gomeni, F.M.M.; Deleuze-Masquéfa, C. Liquid chromatography-electrospray ionization-tandem mass spectrometry method for quantitative estimation of new imiqualine leads with potent anticancer activities in rat and mouse plasma. Application to a pharmacokinetic study in mice. J. Pharm. Biomed. Anal. 2018, 148, 369-379. [CrossRef] [PubMed]

13. Wood, D.C.; Weber, F.S.; Palmquist, M.A. Continued studies in the toxicology of dimethyl sulfoxide (DMSO). J. Pharmacol. Exp. Ther. 1971, 177, 520-527. [PubMed]

14. Abet, V.; Filace, F.; Recio, J.; Alvarez-Builla, J.; Burgos, C. Prodrug approach: An overview of recent cases. Eur. J. Med. Chem. 2017, 127, 810-827. [CrossRef] [PubMed]

15. Jornada, D.H.; dos Santos Fernandes, G.F.; Chiba, D.E.; de Melo, T.R.F.; dos Santos, J.L.; Chung, M.C. The Prodrug Approach: A Successful Tool for Improving Drug Solubility. Molecules 2015, 21, 42. [CrossRef] [PubMed]

16. Drag-Zalesinska, M.; Kulbacka, J.; Saczko, J.; Wysocka, T.; Zabel, M.; Surowiak, P.; Drag, M. Esters of betulin and betulinic acid with amino acids have improved water solubility and are selectively cytotoxic toward cancer cells. Bioorg. Med. Chem. Lett. 2009, 19, 4814-4817. [CrossRef] [PubMed]

17. Parra, A.; Rivas, F.; Lopez, P.E.; Garcia-Granados, A.; Martinez, A.; Albericio, F.; Marquez, N.; Muñoz, E. Solution- and solid-phase synthesis and anti-HIV activity of maslinic acid derivatives containing amino acids and peptides. Bioorg. Med. Chem. 2009, 17, 1139-1145. [CrossRef] [PubMed]

18. Jeong, H.J.; Chai, H.B.; Park, S.Y.; Kim, D.S. Preparation of amino acid conjugates of betulinic acid with activity against human melanoma. Bioorg. Med. Chem. Lett. 1999, 9, 1201-1204. [CrossRef]

19. Schmeda-Hirschmann, G.; Rodríguez, J.A.; Theoduloz, C.; Valderrama, J.A. Gastroprotective effect and cytotoxicity of labdeneamides with amino acids. Planta Med. 2011, 77, 340-345. [CrossRef] [PubMed]

20. Lu, X.-M.; Yi, H.-W.; Xu, J.-L.; Sun, Y.; Li, J.-X.; Cao, S.-X.; Xu, Q. A novel synthetic oleanolic acid derivative with amino acid conjugate suppresses tumour growth by inducing cell cycle arrest. J. Pharm. Pharmacol. 2007, 59, 1087-1093. [CrossRef] [PubMed]

21. Zghaib, Z.; Guichou, J.-F.; Vappiani, J.; Bec, N.; Hadj-Kaddour, K.; Vincent, L.-A.; Paniagua-Gayraud, S.; Larroque, C.; Moarbess, G.; Cuq, P.; et al. New imidazoquinoxaline derivatives: Synthesis, biological evaluation on melanoma, effect on tubulin polymerization and structure-activity relationships. Bioorg. Med. Chem. 2016, 24, 2433-2440. [CrossRef] [PubMed]

22. Testa, B.; Crivori, P.; Reist, M.; Carrupt, P.-A. The influence of lipophilicity on the pharmacokinetic behavior of drugs: Concepts and examples. Perspect. Drug Discov. Des. 2000, 19, 179-211. [CrossRef] 
23. Henchoz, Y.; Bard, B.; Guillarme, D.; Carrupt, P.-A.; Veuthey, J.-L.; Martel, S. Analytical tools for the physicochemical profiling of drug candidates to predict absorption/distribution. Anal. Bioanal. Chem. 2009, 394, 707-729. [CrossRef] [PubMed]

24. Box, K.J.; Comer, J.E.A. Using measured $\mathrm{pKa}, \operatorname{LogP}$ and solubility to investigate supersaturation and predict BCS class. Curr. Drug Metab. 2008, 9, 869-878. [CrossRef] [PubMed]

25. Avdeef, A. Physicochemical profiling (solubility, permeability and charge state). Curr. Top. Med. Chem. 2001, 1, 277-351. [CrossRef] [PubMed]

26. Remko, M.; Remková, A.; Broer, R. A Comparative Study of Molecular Structure, pKa, Lipophilicity, Solubility, Absorption and Polar Surface Area of Some Antiplatelet Drugs. Int. J. Mol. Sci. 2016, 17, 388. [CrossRef] [PubMed]

27. Fridgeirsdottir, G.A.; Harris, R.; Fischer, P.M.; Roberts, C.J. Support Tools in Formulation Development for Poorly Soluble Drugs. J. Pharm. Sci. 2016, 105, 2260-2269. [CrossRef] [PubMed]

Sample Availability: Samples of the compounds are not available from the authors.

(C) 2018 by the authors. Licensee MDPI, Basel, Switzerland. This article is an open access article distributed under the terms and conditions of the Creative Commons Attribution (CC BY) license (http://creativecommons.org/licenses/by/4.0/). 